\title{
Fluorescence nanoscopy in three dimensions
}

\author{
Dissertation \\ zur Erlangung des Doktorgrades \\ der Mathematisch-Naturwissenschaftlichen Fakultäten \\ der Georg-August-Universität zu Göttingen
}

vorgelegt von

Claudia Geisler

aus Hannover

Göttingen, 2009 
D7

Referent: Prof. Dr. Rainer G. Ulbrich

Korreferent: Prof. Dr. Dr. h. c. Stefan W. Hell

Tag der mündlichen Prüfung: 15.12.2009 


\begin{abstract}
Live-cell microscopy faces two challenges when neither the spatial nor the temporal information about the specimen should be lost. First, the microscope must be able to resolve the smallest structure to be investigated and, second, the imaging speed must be at least on the time-scale of the ongoing dynamics. This thesis expands two superresolution concepts, namely 4Pi and single marker switching (SMS) microscopy, and addresses both problems. A fast and signal-efficient 4Pi multifocal multiphoton microscope is introduced which combines an unprecedented frame acquisition of up to the video rate $(25 \mathrm{~Hz})$ with a 6-fold improvement in the axial resolution compared to two-photon microscopy. This work further establishes a novel imaging protocol for SMS microscopy, which cuts down the recording times 100-fold to 2-2.5 minutes compared to previous implementations and enables two-dimensional imaging in the interior of intact cells. By additionally employing two offset detection channels for the axial marker position estimation, threedimensional (3D) SMS imaging of a volume of up to $1.2 \mu \mathrm{m}$ in depth is rendered possible with high linearity and without any need for axial scanning. The positioning accuracy for the marker from which ca. 1600 photons are detected is 16-fold (laterally) and 12-fold (axially) better than the resolution of a conventional confocal microscope. The potential of both concepts for the life sciences is demonstrated by imaging 3D structures in living cells and analyzing their spatial changes with time.
\end{abstract}

\title{
Kurzdarstellung
}

Die mikroskopische Untersuchung von Prozessen auf der submikrometer Skala birgt zwei grundsätzliche Herausforderungen, wenn weder räumliche noch zeitliche Informationen über die Probe verloren gehen sollen. Zum einen muss das Auflösungsvermögen des Mikroskops besser als die kleinste zu untersuchende Struktur sein und zum anderen muss die Aufnahmegeschwindigkeit in der Größenordnung der ablaufenden Prozesse liegen. Diese Arbeit behandelt zwei hochauflösende Fernfeld-Mikroskopieverfahren, namentlich 4Pi-Mikroskopie und Einzelmolekülschaltmikroskopie (englisch: single marker switching, SMS), unter Berücksichtigung dieser beiden Aspekte. Zunächst wird ein schnelles und signaleffizientes multifokales Multiphotonen-4Pi-Mikroskop vorgestellt, welches eine bisher beispiellose Bildaufnahmerate von bis zu $25 \mathrm{~Hz}$ mit einer im Vergleich zur Zweiphotonenmikroskopie sechsfach höheren axialen Auflösung verbindet. Des Weiteren wird ein neues SMS-Mikroskop vorgestellt, welches die Aufnahmezeit um einen Faktor 100 auf 2-2,5 Minuten verkürzt und erstmals eine zweidimensionale Bildgebung innerhalb intakter Zellen ermöglicht. Die zusätzliche Erweiterung auf zwei versetzte Detektionskanäle zur Bestimmung der axialen Markerposition erlaubt zudem eine dreidimensionale Bildgebung in einem Volumen von bis zu 1,2 $\mu$ m Tiefe mit hoher Linearität und ohne dass die Probe in axialer Richtung gescannt werden muss. Die Positionsbestimmung eines Markers, von dem ca. 1600 Photonen detektiert werden, ist lateral 16-fach und axial 12-fach besser als die Auflösung eines herkömmlichen Konfokalmikroskops. Anhand von Aufnahmen dreidimensionaler Strukturen innerhalb lebender Zellen sowie der Analyse ihrer zeitlichen Veränderung wird das Potential, welches beide Mikroskopieverfahren für die Lebenswissenschaften bieten, verdeutlicht. 



\section{Abbreviations}

$\begin{array}{ll}\text { 1P } & \text { one-photon } \\ \text { 2P } & \text { two-photon } \\ \text { 2D } & \text { two-dimensional } \\ \text { 3D } & \text { three-dimensional } \\ \text { 3(5)p } & \text { three (five) point } \\ \text { AFM } & \text { atomic force microscope } \\ \text { DNA } & \text { deoxyribonucleic acid } \\ \text { (e)GFP } & \text { (enhanced) green fluorescent protein } \\ \text { (EM)CCD } & \text { (electron multiplying) charge-coupled device } \\ \text { FPALM } & \text { fluorescence photoactivation localization microscopy } \\ \text { FWHM } & \text { full-width-at-half-maximum } \\ \text { MMM (4Pi) } & \text { (4Pi) multifocal multiphoton microscopy } \\ \text { OTF } & \text { optical transfer function } \\ \text { PALM(IRA) } & \text { photoactivated localization microscopy (with independently running acquisition) } \\ \text { PBS } & \text { phosphate buffered saline } \\ \text { PSF } & \text { point spread function } \\ \text { PVA } & \text { polyvinyl alcohol } \\ \text { RESOLFT } & \text { reversible saturable optical (fluorescence) transitions } \\ \text { RSFP } & \text { reversibly switchable fluorescent protein } \\ \text { SMS } & \text { single marker switching } \\ \text { SNR } & \text { signal-to-noise ratio } \\ \text { SRA577(545) } & \text { switchable rhodamine amide with emission maximum at 577(545) nm } \\ \text { STED } & \text { stimulated emission depletion } \\ \text { STORM } & \text { stochastic optical reconstruction microscopy } \\ \text { Ti:Sa } & \text { Titanium:Sapphire } \\ \text { TIRF } & \text { total internal reflection fluorescence } \\ \text { UV } & \text { ultraviolet } \\ \end{array}$





\section{Contents}

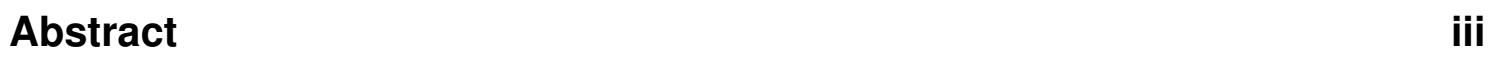

Abbreviations v v

Contents vii

1 Introduction 1

2 Conventional fluorescence microscopy and its resolution 5

2.1 Introduction to fluorescence $\ldots \ldots \ldots \ldots \ldots \ldots$

2.2 Introduction to microscopy . . . . . . . . . . . . . . . . 7

2.2 .1 Image formation . . . . . . . . . . . . . . . . 7

2.2.2 Epifluorescence microscopy . . . . . . . . . . . . . . 10

2.2 .3 Confocal microscopy . . . . . . . . . . . . . . . 12

2.2.4 Two-photon microscopy . . . . . . . . . . . . . . . . . . . 14

3 4Pi multifocal multiphoton microscopy 17

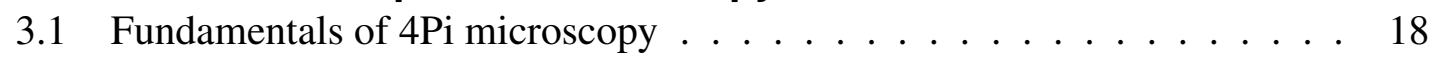

3.1.1 Multifocal illumination in the 4Pi microscope . . . . . . . . . 22

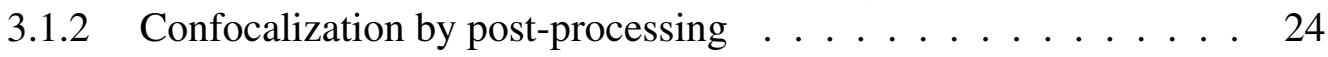

3.2 Experimental implementation . . . . . . . . . . . . . . . . 25

3.3 Experimental results for two recording schemes . . . . . . . . . 30

4 Single marker switching microscopy 37

4.1 Introduction to high resolution switching-based microscopy . . . . . . . 37

4.2 Breaking the diffraction barrier by switching single markers $\ldots \ldots .38$

4.2 .1 Photon statistics . . . . . . . . . . . . . . . . . 41

4.2 .2 Positioning accuracy $\ldots \ldots \ldots \ldots \ldots \ldots$

4.2 .3 Image representation . . . . . . . . . . . . . . . 43

4.3 SMS microscopy in two dimensions . . . . . . . . . . . . . . 44

4.3.1 Imaging protocol with independently running acquisition . . . . 45

4.3.2 Single molecule spectroscopy . . . . . . . . . . . . . . 46

4.3 .3 Experimental implementation . . . . . . . . . . . . . . . 48

4.3.4 Positioning in the lateral direction . . . . . . . . . . . . . 49

4.3.5 High resolution 2D images of technical and biological specimens 50

4.4 Extension to the third dimension . . . . . . . . . . . . . . . . 54 
4.4.1 Axial positioning - defocus method . . . . . . . . . . . 55

4.4.2 Imaging setup and image registration .......... . . 56

4.4.3 3D positioning algorithm . . . . . . . . . . . . 60

4.4.4 Experimental results for 3D imaging . . . . . . . . . . . . . 64

4.5 Applications of 3D SMS microscopy . . . . . . . . . . . . . . . 68

4.5.1 Structural analysis of colloidal crystals . . . . . . . . . . 69

4.5.2 In-vivo measurements: structural changes with time . . . . . . 71

5 Summary and outlook $\quad 77$

$\begin{array}{ll}\text { Bibliography } & 81\end{array}$

A Protocols for sample preparation $\quad 91$

$\begin{array}{ll}\text { List of publications } & 95\end{array}$

$\begin{array}{lr}\text { Acknowledgements } & 97\end{array}$ 


\section{Introduction}

In the course of the past decades, far-field fluorescence microscopy has emerged as one of the most powerful and versatile tools in the life sciences and beyond. It is exceptional in its ability to non-invasively image the interior of translucent specimens in three dimensions. Further, specific tagging or labeling of target proteins or cellular compartments with fluorescent markers lends an exceptional molecular specificity to the method. Even more important, far-field fluorescence microscopy enables the imaging of live cells since it is non-destructive and compatible with live-cell environments. However, for many decades, the resolution of its standard variants, like for example epifluorescence microscopy and confocal microscopy, has been limited by diffraction. This means that the microscope's ability to distinguish closely positioned objects is restricted by the fact that any point-like object is not imaged to a point, but to an extended elliptical intensity distribution. This diffraction barrier, which was discovered by Ernst Abbe in 1873 [1], limits the smallest distance between two objects at which they can still be distinguished to

$$
\Delta r \approx 0.5 \cdot \frac{\lambda_{0}}{n \sin (\alpha)} \quad \text { and } \quad \Delta z \approx 2 \cdot \frac{\lambda_{0}}{n \sin ^{2}(\alpha)}
$$

in the lateral $\left(x, y ; r=\sqrt{x^{2}+y^{2}}\right)$ and in the axial (z) directions, respectively. Here, $\lambda_{0}$ denotes the vacuum wavelength of light, $n$ is the refractive index of the embedding medium and $\alpha$ is the semi-aperture angle of the objective lens. This resolution limit cannot be improved arbitrarily. The semi-aperture angle of the objective lens is presently limited by technical issues to about $74^{\circ}$. Likewise, the refractive index cannot be increased at will because a large refractive index mismatch between the immersion medium and the embedding medium leads to spherical aberrations [2]. For excitation light in the visible spectrum between 450 and $700 \mathrm{~nm}$, the main point spread function (PSF) maximum has a diameter of $>200 \mathrm{~nm}$ in the focal plane $(x, y)$ and a diameter of $>500 \mathrm{~nm}$ along the optic axis (z). A reduction of the wavelength further down to the ultraviolet (UV) range is hardly compatible with live-cell imaging due to the photo damage induced by photons of high energy. Furthermore, biological specimens typically show a significant UV light induced autofluorescence. In addition, conventional lens-based optics, whose dispersion significantly increases for UV light, is optimized for the visible range. Therefore, expensive UV optics like for example objective lenses with chromatic aberrations compensated for UV light would be necessary.

In order to overcome these limits, microscopy techniques have emerged whose aim was to shrink the effective size of the PSF. By coherently adding the wavefronts of two opposing lenses, 4Pi microscopy [3] [4] and the related concepts $\mathrm{I}^{5} \mathrm{M}$ [5] and its extension 
$I^{5} S$ [6] have reduced the main maximum of the PSF by a factor of $3-7$ along the optic axis. These concepts essentially expanded the solid aperture angle in microscopy and are thus still subject to Abbe's law.

A more fundamental improvement has been achieved by exploiting the molecular transitions of the marker [7] [8]. This goes beyond just using the marker as a mere contrast agent and allows one to switch the marker between a bright signaling state and a dark non-signaling state [9] [10]. The first method which made use of this and fundamentally broke the diffraction barrier, is STED microscopy (stimulated emission depletion microscopy) that, in principle, offers unlimited resolution [11]. STED microscopy employs the transition from the fluorescent singlet state to the dark ground state, the excited state is depleted by stimulated emission. The same states are used in saturated pattern excitation microscopy (SPEM), which is also called saturated structured illumination microscopy (SSIM) [12] [13] [14]. Since any molecular transition which is a reversible saturable optical (fluorescent) transition (RESOLFT) can be employed for breaking the diffraction barrier [15], other intramolecular marker transitions have also been used. The resolution was enhanced beyond the diffraction limit, for instance, by depleting the ground state [16] [17] and by reversibly switching a marker between two conformational states or other optically bistable transitions [18].

Molecular switching has also paved the way for powerful superresolution techniques that switch markers stochastically and individually in a wide-field illumination microscope. This scheme has been referred to as PALM (photoactivated localization microscopy) [19], FPALM (fluorescence photoactivation localization microscopy) [20], STORM (stochastic optical reconstruction microscopy) [21] among others and will be called by the hypernym SMS (single marker switching) microscopy in this thesis. SMS microscopy is based on the same principle as the aforementioned techniques within the RESOLFT concept. It uses a transition between a dark state and a bright state to switch fluorophores on and off sequentially in time [10]. Within the SMS concept, the switching is used to stochastically separate identical neighboring markers in space and time so as to unambiguously assign a bunch of photons to one marker and subsequently mathematically estimate its position.

In this thesis, both the 4Pi concept and the SMS concept will be explored and expanded, hereby focusing on their applicability to the life sciences. The imaging of living biological specimens typically poses two challenges. First, the main advantage of imaging living cells as opposed to dead specimens is that they not only disclose their structure but also their dynamics. Thus, the image acquisition time should be at least on the time-scale of the processes under investigation or even faster. Otherwise, either interesting details escape the microscopist's attention or, even worse, the structures appear distorted by the ongoing dynamics. Second, the full directional (superresolution) information about the dynamics is only accessible if the microscope furnishes not only two-dimensional (2D) information, but also provides superresolution in the remaining axial direction.

In the case of $4 \mathrm{Pi}$ microscopy the emphasis will be laid on improving the image acquisition rate. Though stunning advances have been reported concerning the shape of the 
PSF [22] [23], these are accompanied by long 2D image acquisition times in the order of 10 seconds [24]. A 4Pi multifocal multiphoton microscope (MMM 4Pi) will be presented, which significantly improves the image acquisition time compared to previous MMM 4Pi implementations [25] and enables video rate recording.

In the case of SMS microscopy, both aforementioned challenges will be tackled. A novel image acquisition protocol will be introduced which speeds up the recording time, simplifies the setup and enables imaging in the depth of the specimen, the latter being an indispensable prerequisite for three-dimensional (3D) imaging. A concept for 3D positioning will be presented along with its experimental performance. As an initial study, 3D SMS visualizes the motion of parts of the cytoskeleton at an unprecedented resolution and proves its feasibility beyond the life sciences, in particular to the structural analysis of self-assembled colloidal crystals.

This thesis is structured as follows. Subsequent to this general motivation, a detailed introduction to the basics of fluorescence and to the resolution problem in conventional microscopy will be given in chapter 2 . Chapter 3 provides the relevant basics of $4 \mathrm{Pi}$ microcopy, illustrates the research performed within the framework of this thesis and shows the application to live-cell imaging. Chapter 4 introduces the concept of SMS microscopy in detail and presents the advances concerning $2 \mathrm{D}$ and $3 \mathrm{D}$ imaging. The method will be introduced in two particular fields of application. The thesis is concluded by a general summary and discussion in chapter 5 . 



\section{Conventional fluorescence microscopy and its resolution}

The far-field imaging techniques discussed in this thesis rely on the detection of fluorescence for investigating structure and dynamics of biological systems or matter on the nanoscale. The following section therefore provides a basic description of the fluorescence process in a standard fluorophore [26] [27].

\subsection{Introduction to fluorescence}

The Jablonski diagram in figure 2.1 (a) is a convenient way to visualize intramolecular excitation and de-excitation pathways. It gives a simplified presentation of the relative positions of the energy levels and the transitions among them. However, it does not provide any information about the relative positions of the nuclei.

At first glance, it becomes obvious that the states can be classified into two systems: the singlet system denoted $\mathrm{S}$ and the triplet system denoted $\mathrm{T}$. The thick black lines in figure 2.1 (a) depict the lowest vibrational level of each state, while the gray lines illustrate the levels with higher vibrational energies of the corresponding state. The singlet system is characterized by two electrons of opposite spin ("paired" electrons). Its total spin quantum number $\mathrm{S}=\sum s_{i}$ with $\mathrm{s}_{i}=-\frac{1}{2}$ or $+\frac{1}{2}$ is equal to zero, its multiplicity $\mathrm{M}=2 \mathrm{~S}+1$ is therefore equal to unity. At room temperature, a majority of the molecules are in the lowest vibrational energy level of the ground state $S_{0}$. After absorption of one photon with sufficient energy or of two photons of approximately half the energy, an electronic transition occurs most likely to higher vibrational levels of the first excited state $S_{1}$. The molecule then relaxes non-radiatively to the lowest vibrational level. From there, it can undergo a singlet-singlet transition to vibrational states of $S_{0}$ via a non-radiative (vibrational) process or by emitting radiation. The former process is called "internal conversion" meaning a non-radiative transition between states of the same multiplicity. It is marked by a gray wiggly line in figure 2.1 (a). The latter process is the spontaneous emission of a photon ("fluorescence"). The molecule stays in $\mathrm{S}_{1}$ for a certain period of time before emitting radiation. The average time period depends on the molecule and the environment and is characteristic for the fluorescence process ("fluorescence lifetime"). Fluorescence typically occurs at lower frequencies than the incident one-photon (1P) radiation because of the energy loss during the vibrational relaxation in the first excited state and the transition to higher vibrational energy levels of $S_{0}$. The resulting redshift of the emission spectrum compared to the absorption spectrum is called Stokes-shift. The shape of the spectra is 
(a)

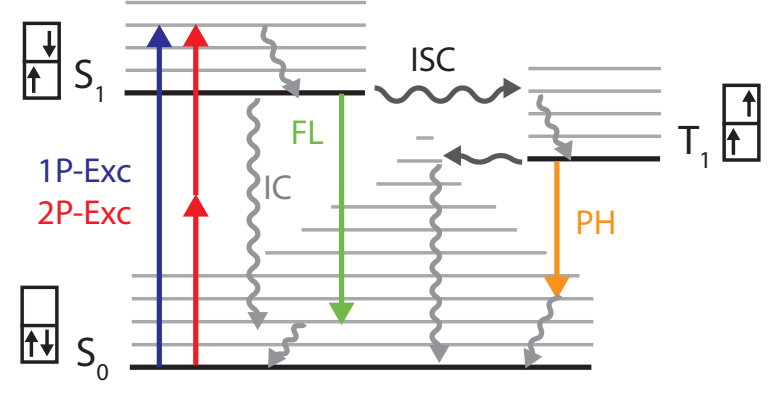

(b)

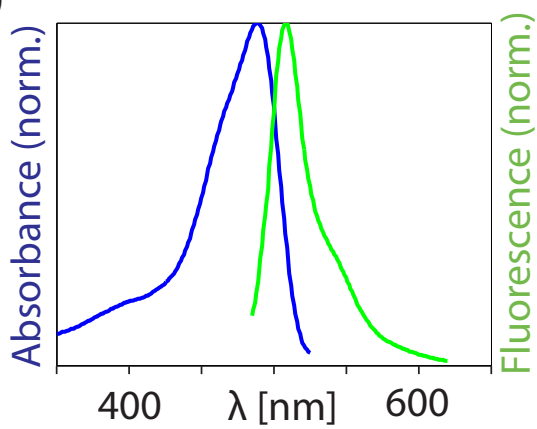

Figure 2.1: (a) Jablonski diagram for a standard fluorophore. Black lines depict the lowest vibrational energy levels of the singlet ground state $S_{0}$, the excited singlet state $S_{1}$ and the excited triplet state $\mathrm{T}_{1}$, while gray lines illustrate the vibrational sub-levels. The respective spin configuration is illustrated by the pairs of arrows. The electronic transition from the lowest vibrational state of $S_{0}$ to higher vibrational levels of $S_{1}$ can be induced by absorption of one photon (blue arrow, 1P-Exc) or by simultaneous absorption of two photons (red arrows, 2P-Exc). Relaxation to the lowest level of $S_{1}$ takes place quickly by internal conversion (light gray wiggly arrow, IC). Possible de-excitation pathways from there are a non-radiative transition to $S_{0}$ via internal conversion, emission of fluorescence (green arrow, FL) or intersystem crossing (dark gray wiggly arrow, ISC) to $T_{1}$ followed by internal conversion to the ground state or by emission of phosphorescence (orange arrow, PH). (b) Normalized absorption and emission spectra of eGFP.

influenced by homogeneous and inhomogeneous broadening due to the continuous set of vibrational levels in each electronic state and due to fluctuations in the solvent environment. As an example, figure 2.1 (b) shows the absorption and emission spectra of the enhanced green fluorescent protein (eGFP).

A further possible transition is the crossing from $S_{1}$ to $T_{1}$ of the triplet system. This system is characterized by two electrons with parallel spins with $S=1$ and $M=3$, thus corresponding to three states of equal energy. These states have lower energy than the singlet state of the same configuration ("Hund's rule"). The crossing between states of different multiplicity which implies a spin flip is forbidden by the spin-conversion rule. However, it is not the total spin quantum number $\mathrm{S}$ which must be conserved, but the total angular momentum $\mathrm{J}=\mathrm{L}+\mathrm{S}$ where $\mathrm{L}$ denotes the angular momentum. Thanks to a weak spin-orbit coupling, the wave function of a singlet state always contains a small admixture of the triplet wave function. A transition between the systems may therefore take place. Intersystem crossing is the non-radiative transition between states of equal energy and different multiplicity. After relaxation to the lowest vibrational level, the transition back to the singlet system is again subject to the spin-conversation rule. This explains why the triplet state $T_{1}$ has a comparatively long lifetime. A further intersystem crossing $T_{1} \rightarrow S_{0}$ which is followed by vibrational relaxation to the lowest vibrational level of $S_{0}$ is usually dominant over the radiative transition between $\mathrm{T}_{1}$ and $\mathrm{S}_{0}$ ("phosphorescence").

After having discussed the transitions which result in emission of fluorescence, it is 
instructive to briefly look into the processes which lead to the irreversible loss of the fluorophore's ability to fluoresce ("photobleaching") [28]. Although photobleaching is poorly-understood in general, the main reason is supposed to involve photodynamic interactions between excited fluorophores and molecular oxygen. When a molecule reaches the metastable triplet state $T_{1}$, it has plenty of time ( $m s$ to $s$ ) to interact with its environment, in particular with molecular oxygen in its triplet ground state. The interaction between fluorophore triplets and molecular oxygen may lead to a generation of singlet oxygen whose decay can create several types of damaging oxygen free radicals. Another cause for photobleaching is probably the fact that fluorophores in their excited triplet state are highly reactive and may undergo irreversibly chemical reactions with other organic molecules. Consequently, photobleaching depends both on the fluorophore itself and on its environment.

\subsection{Introduction to microscopy}

In the following sections, the basics of image formation as well as the implementations of typical conventional microscopes will be presented [29][30][31][32][33]. It should be noted that generally two types of imaging must be distinguished: coherent and incoherent. Since this thesis only deals with fluorescence imaging, in which the phase of the illuminating light is lost during the incoherent fluorescence emission, the incoherent case will be implicitly assumed.

\subsubsection{Image formation}

Let a point-like light source $\mathrm{S}$ be imaged to point $\mathrm{F}$ by an ideal lens. This point source can be for example a single fluorophore whose fluorescence is imaged onto a detector. Due to the wave nature of light, its image is blurred to a 3D distribution around $\mathrm{F}$ which is depicted in figure 2.2 (a-c). The electric field distribution at $\vec{r}=(x, y, z)$ is called the amplitude PSF and can be described according to the 3D scalar diffraction theory using the Fresnel approximation [29][34]:

$$
h_{A}(\vec{r})=A \int_{0}^{\alpha} \sqrt{\cos (\theta)} \sin (\theta) J_{0}\left(k \sqrt{x^{2}+y^{2}} \sin (\theta)\right) \exp (i k z \cos (\theta)) d \theta .
$$

$A$ denotes a normalization constant, $J_{0}$ is the zero order Bessel function of first kind, $\theta$ denotes the polar aperture angle and $\alpha$ is its maximum. The wave number $k=2 \pi n / \lambda_{0}$ depends on the refractive index $n$ and on the vacuum wavelength $\lambda_{0}$.

Since most electronic detectors see the intensity rather than the electric field, it is instructive to define the intensity distribution or (intensity) PSF which is given by the square of the absolute value of the amplitude PSF: 
(a)

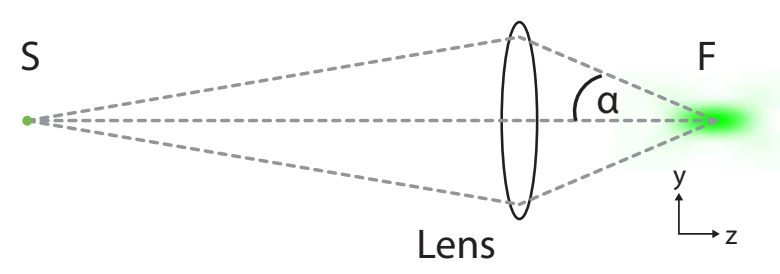

(b)

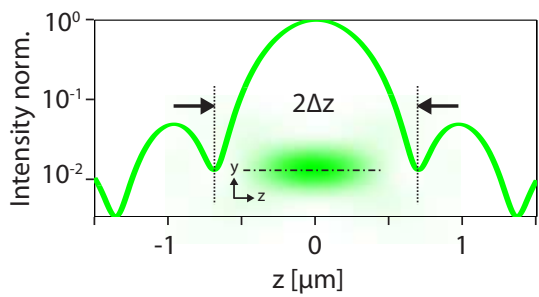

(c)

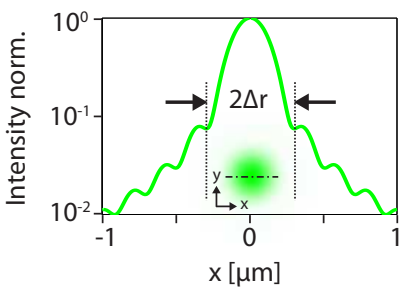

Figure 2.2: The PSF. (a) A point-like source $S$ is blurred to a 3D intensity distribution around $\mathrm{F}$, the PSF, when being imaged by a lens. $\alpha$ denotes the semi-aperture angle of the lens. (b) Semi-logarithmic plot of the normalized axial intensity profile of the PSF along the dash-dotted line. The shape of the PSF in its center yz plane is shown in the bottom. The axial distance of the first minima (marked by the arrows) is a measure for the axial resolution $\Delta z$ in (2.6). (c) Semilogarithmic plot of the normalized lateral intensity profile of the PSF along the dash-dotted line. The shape of the PSF in its center xy plane is shown in the bottom. Half the distance between the first two minima is a measure for the lateral resolution $\Delta r$ in (2.5). The intensity profiles exhibit a strong main maximum and progressively weaker minima and maxima.

$$
h(\vec{r})=\left|h_{A}(\vec{r})\right|^{2} .
$$

Altogether, the PSF of the imaging system describes how a point in the object space looks like in the image space. This image construction can be expanded from one single point source to a 3D self-luminous object (e.g. a distribution of fluorophores) by integrating the images of every single point of the object. The image $I(\vec{r})$ of an object $O(\vec{r})$ is therefore given by the convolution $(\otimes)$ of the object with the PSF $h(\vec{r})$ [30]:

$$
I(\vec{r})=\int O\left(\vec{r}^{\prime}\right) \cdot h\left(\vec{r}-\vec{r}^{\prime}\right) d \vec{r}^{\prime}=O(\vec{r}) \otimes h(\vec{r}) .
$$

Note that the object is blurred by the PSF. In order to create a sharp image, it is necessary that the PSF is as well-localized as possible, which is usually the case for objective lenses with high numerical aperture.

The imaging process which is characterized by the PSF in the spatial domain can also be considered from the frequency domain viewpoint. Here, the object and the image are described by their spatial frequencies. The imaging system is therefore characterized by its transmittance of spatial frequencies. Fourier transformation of equation (2.3) and application of the convolution theorem yields 


$$
\mathcal{F}(I(\vec{r}))=\mathcal{F}(O(\vec{r}) \otimes h(\vec{r}))=\mathcal{F}(O(\vec{r})) \cdot \mathcal{F}(h(\vec{r})) .
$$

The frequency spectrum of the image $\mathcal{F}(I(\vec{r}))$ is thus given by the product of the frequency spectrum of the object $\mathcal{F}(O(\vec{r}))$ and the Fourier transform of the PSF $\mathcal{F}(h(\vec{r}))$. If $\mathcal{F}(h(\vec{r}))$ has very small or zero values for particular spatial frequencies, these frequencies are very badly transmitted or not transmitted at all by the microscope. Consequently, details of the object which are described by these spatial frequencies cannot be resolved in the image. The normalized Fourier transform of the PSF is usually referred to as the optical transfer function (OTF).

\section{A measure for resolution}

The PSF given by equation (2.2) is cylindrically symmetric and its maximum resides at the focal point $\mathrm{F}$ at the coordinates $\vec{r}=0$. Figure 2.2 (b) presents the intensity profile along the optic axis and shows its elongation in the $\mathrm{z}$ direction. The intensity profile in the focal plane features a pronounced main maximum and progressively weaker minima and maxima which can be easily seen in the semi-logarithmic plot in figure 2.2 (c). This pattern is referred to as Airy pattern and the central region which extends to the first zero is known as the Airy disk. According to the Rayleigh criterion, two objects can still be separated if the main maximum of one image falls into the first minimum of the other image. The Airy disk's radius serves therefore as a measure for the resolution $\Delta r$ in the lateral direction:

$$
\Delta r=0.61 \frac{\lambda_{0}}{\mathrm{NA}} .
$$

It should be noted again that the Rayleigh criterion relies on the assumption that the two point sources radiate incoherently. If the two sources emit coherent light, their complex amplitude PSFs rather than their (intensity) PSFs must be considered. In the latter case, the resolution decreases in general and the Rayleigh criterion is inadequate. ${ }^{1}$

Analogous to $\Delta r$, the resolution in the axial direction $\Delta z$ can be defined to be half the distance between the first minima:

$$
\Delta z=2.00 \frac{n \lambda_{0}}{\mathrm{NA}^{2}}
$$

$\Delta z$ is also called depth of field and defines the axial distance from the focal point $\mathrm{F}$ at which the image quality is still acceptable.

The Airy pattern is often approximated by a Gaussian which fits the main maximum quite well and only omits the weak rings. The resolution can then be conveniently defined by its full-width-at-half-maximum (FWHM):

$$
\widetilde{\Delta r}=0.51 \frac{\lambda_{0}}{\mathrm{NA}}
$$

In the coherent case, the Sparrow criterion gives an adequate measure for the lateral resolution [32]. 


$$
\widetilde{\Delta z}=1.77 \frac{n \lambda_{0}}{\mathrm{NA}^{2}}
$$

\section{A measure for optical sectioning}

A good benchmark for the optical sectioning capability of an imaging system is its zresponse, which corresponds to the detected fluorescence emitted by an infinitesimal thin layer in the xy plane whose position is scanned along the $\mathrm{z}$ axis:

$$
I_{Z}(z)=\int h(\vec{r}) d x d y
$$

If a microscope system is capable of optical sectioning, its z-response rapidly decreases for increasing distance between the fluorescent layer and the focal plane.

The sea-response is a second measure for the optical sectioning capability. It corresponds to the signal of a fluorescent half-space which is scanned along the optic axis:

$$
I_{\text {sea }}(z)=\int_{-\infty}^{z} I_{Z}(z) d z
$$

Strictly speaking, $I_{\text {sea }}(z)$ does not provide more information about the system's properties than the z-response does. However, it nicely illustrates the contribution of local side bands of the PSF to the detected signal which especially gains in importance when imaging thick samples.

In order to compare experimental data with theoretical results, it is usually appropriate to analyze the sea-response of a fluorescent layer of finite thickness D:

$$
I_{\text {sea }}(z, D)=\int_{-z+\frac{D}{2}}^{-z+\frac{D}{2}} I_{Z}(z) d z .
$$

In the following, three particular types of conventional microscopes, their typical experimental implementations and their imaging properties will be discussed.

\subsubsection{Epifluorescence microscopy}

The epifluorescence microscope separates the fluorescence and illumination light elegantly by exciting and detecting from the same side of the sample. A typical setup is shown in figure 2.3. The 1P excitation light is collimated and reflected by a dichroic mirror. This mirror reflects light below a particular cut-off wavelength and transmits longer wavelengths. It therefore acts as a long-pass filter. The sample to be investigated sits in the focal plane of the objective lens and is usually mounted on a glass cover slip which most objective lenses are corrected for. The illumination light is focused on the back-focal plane of the objective lens. The resulting homogeneous illumination of the sample is often 


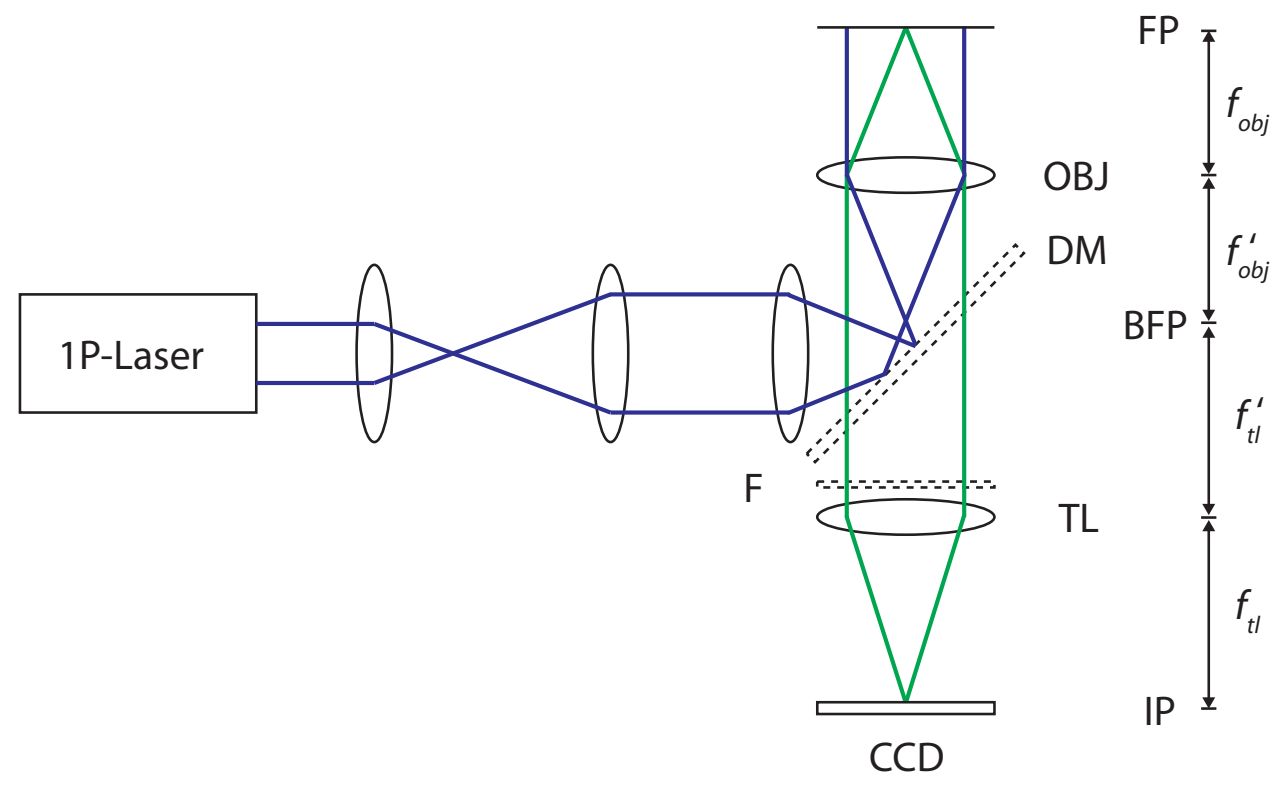

Figure 2.3: Typical epifluorescence microscope setup with a laser source for $1 \mathrm{P}$ excitation (1PLaser). The excitation light (blue) is collimated and focused onto the back-focal plane (BFP) of the objective lens (OBJ) with focal length $\mathrm{f}_{\text {obj. }}$. This results in a homogeneous illumination of the focal plane (FP). The fluorescence (green) is collected by the same lens, separated from the laser light by a dichroic mirror (DM) and imaged onto a camera (CCD) by a tube lens (TL) with focal length $\mathrm{f}_{\mathrm{tl}}$. A band-pass filter $(\mathrm{F})$ suppresses background light outside the fluorescence spectrum. IP: image plane.

referred to by the term wide-field illumination. The emitted fluorescence is collected by the same objective lens. The fluorescence spectrum is redshifted with respect to the excitation light due to the Stokes-shift (cf. section 2.1). Most of the fluorescence is therefore transmitted by the dichroic mirror, while backscattered excitation light is reflected which efficiently separates the fluorescence light from the excitation light. Additional band-pass filters are usually inserted into the detection path to remove background light outside the fluorescence spectrum. A real image of the emitter distribution in the sample is formed by a tube lens. The image can either be recorded in the first image plane by a detector or, alternatively, it can be back-projected further to the retina of the experimentalist's eye by an ocular and the human crystalline lens. The PSF $h_{\mathrm{epi}}(\vec{r})$ of the epifluorescence microscope is given by its normalized detection PSF which is given by equation (2.2) for an ideal system and its normalized constant illumination PSF:

$$
h_{\mathrm{epi}}(\vec{r})=h(\vec{r}) .
$$

Figure 2.6 (a) depicts the central xy plane (x, y, z=0) of $h_{\text {epi }}$ for a detection wavelength $\lambda_{\text {det }}=508 \mathrm{~nm}$ which is the emission peak of eGFP (cf. figure 2.1 (b)). Figure 2.6 (e) shows the constant z-response (cf. equation (2.9)), which implies that the epifluorescence microscope does not have any optical sectioning capabilities. 


\subsubsection{Confocal microscopy}

The epifluorescence microscope's drawback is that the signal from the out-of-focus planes is not filtered out. On the contrary, fluorescence from the full depth of the illuminated volume adds to the signal. In his patent in 1957, Marvin Minsky presented the concept for a stage-scanning confocal system [35]. He suggested that a point source is imaged into the sample. The resulting diffraction-limited intensity distribution serves as the illumination PSF $h_{\text {ill }}(\vec{r})$. The fluorescence is collected by the same objective lens, separated from the illumination light by a dichroic mirror (cf. section 2.2.2) and imaged onto a detector like, for example, an avalanche photodiode or a photomultiplier. By placing a pinhole in the (back-projected) image plane, only that fluorescence which stems from the focal plane can reach the detector. The point source, its image in the sample and the detection pinhole are in conjugated planes. The PSF of a confocal system is therefore determined by the normalized illumination PSF $h_{\mathrm{ill}}(\vec{r})$, which describes the probability of photon emission at $\vec{r}$, and the normalized detection PSF $h_{\text {det }}(\vec{r})$, which describes the probability to detect a photon which was emitted at $\vec{r}$ :

$$
h_{\mathrm{conf}}(\vec{r})=h_{\mathrm{ill}}(\vec{r}) \cdot h_{\mathrm{det}}(\vec{r}) .
$$

While $h_{\text {ill }}(\vec{r})$ is determined by the illumination wavelength $\lambda_{\text {ill }}, h_{\text {det }}(\vec{r})$ depends on the detection wavelength $\lambda_{\text {det }}$. Since the ratio $\lambda_{\text {ill }} / \lambda_{\text {det }}$ is usually around 0.95 [36], the confocal PSF can be approximated by

$$
h_{\text {conf }}(\vec{r}) \approx h_{\text {ill }}^{2}(\vec{r}) .
$$

Due to its quadratic dependence on the illumination PSF, the FWHM of $h_{\text {conf }}$ is narrowed down compared to $h_{\text {epi }}$ which leads to a $\sqrt{2}$-fold improvement of the resolution. Additionally, out-of-focus contributions are suppressed which is illustrated by the z-response and the steep sea-response in figure 2.6 (e) and 2.6 (f). The central xy slice (x, y, z=0) of $h_{\text {conf }}$ is shown in figure 2.6 (c) for $\lambda_{\text {ill }}=488 \mathrm{~nm}$ and $\lambda_{\text {det }}=508 \mathrm{~nm}$.

Equation (2.14) is valid for an ideal confocal system in the limiting case of an infinitesimal small detection pinhole. However, in practice, a finite pinhole size is necessary in order to collect sufficient signal. Since light paths are reversible, imaging onto the pinhole can be interpreted as imaging the pinhole into the sample. The image of the pinhole, the detection PSF $h_{\text {det,real }}(\vec{r})$, represents the probability for the detection of a photon which is emitted at $\vec{r}$. If the pinhole is described by the pinhole function

$$
p(\vec{r})=p(x, y, z=0)=\left\{\begin{array}{cc}
1 & \text { for } \sqrt{x^{2}+y^{2}} \leq p_{0} \\
0 & \text { otherwise }
\end{array},\right.
$$

the real detection PSF is given by the 2D convolution $\left(\otimes_{2 \mathrm{D}}\right)$ of the detection PSF $h_{\mathrm{det}}(\vec{r})$ with the pinhole function $p(\vec{r})$ :

$$
h_{\text {det,real }}(\vec{r})=h_{\text {det }}(\vec{r}) \otimes_{2 \mathrm{D}} p(\vec{r}) .
$$




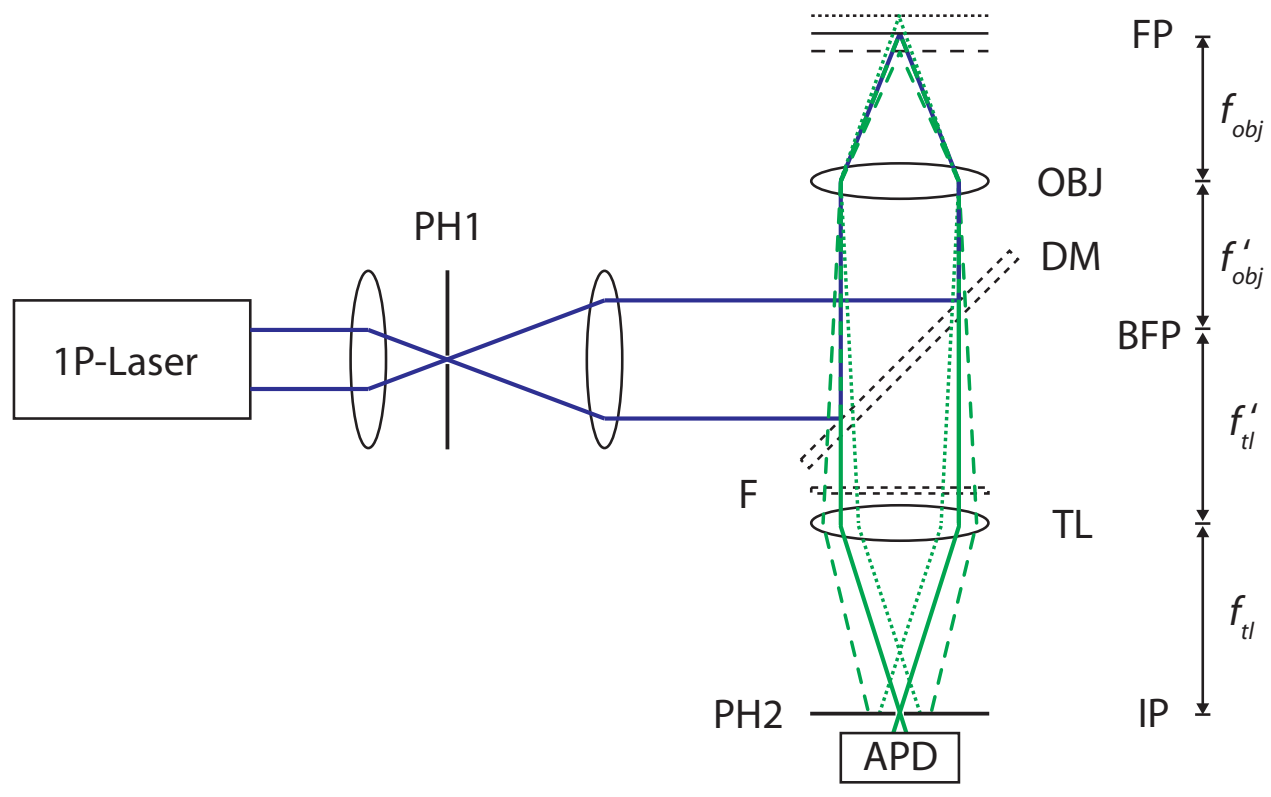

Figure 2.4: Typical confocal microscope setup with a laser source for 1P excitation (1P-Laser). A pinhole (PH1), which is illuminated by the laser light (blue), serves as a point source and is imaged onto the focal plane (FP) of the objective lens (OBJ) with focal length $\mathrm{f}_{\text {obj }}$. Fluorescence (green) is collected by the same lens, separated from the laser light by a dichroic mirror (DM) and imaged onto the image plane (IP) by a tube lens (TL) with focal length $\mathrm{f}_{\mathrm{tl}}$. A detection pinhole ( $\mathrm{PH} 2)$ ensures that only that fluorescence light which originates in the focal plane passes through to the detector (avalanche photodiode, APD). The excitation pinhole, the focal plane and the detection pinhole are in conjugate planes. BFP: back-focal plane, F: fluorescence filter.

The PSF of the real confocal microscope is thus given by

$$
h_{\text {conf,real }}(\vec{r})=h_{\text {ill }}(\vec{r}) \cdot h_{\text {det,real }}(\vec{r}) \text {, }
$$

which is illustrated for $\lambda_{\mathrm{ill}}=488 \mathrm{~nm}, \lambda_{\mathrm{det}}=508 \mathrm{~nm}$ and for the pinhole size $\mathrm{p}_{0}$ of the diameter of the Airy disk in figure 2.6 (d).

In order to record the whole region of interest, the illumination PSF can be moved over the sample by changing the angle of the incident beam in the back-focal plane of the objective. Likewise, the stage can be moved step-by-step through the spot using, for example, a piezo-actuated stage. The step size should be approximately twice as fine as the resolution according to the Nyquist-Shannon criterion [37]. For every position, the recorded intensity and the respective coordinates in the sample are stored to a computer and the final image is afterwards assembled point by point. 


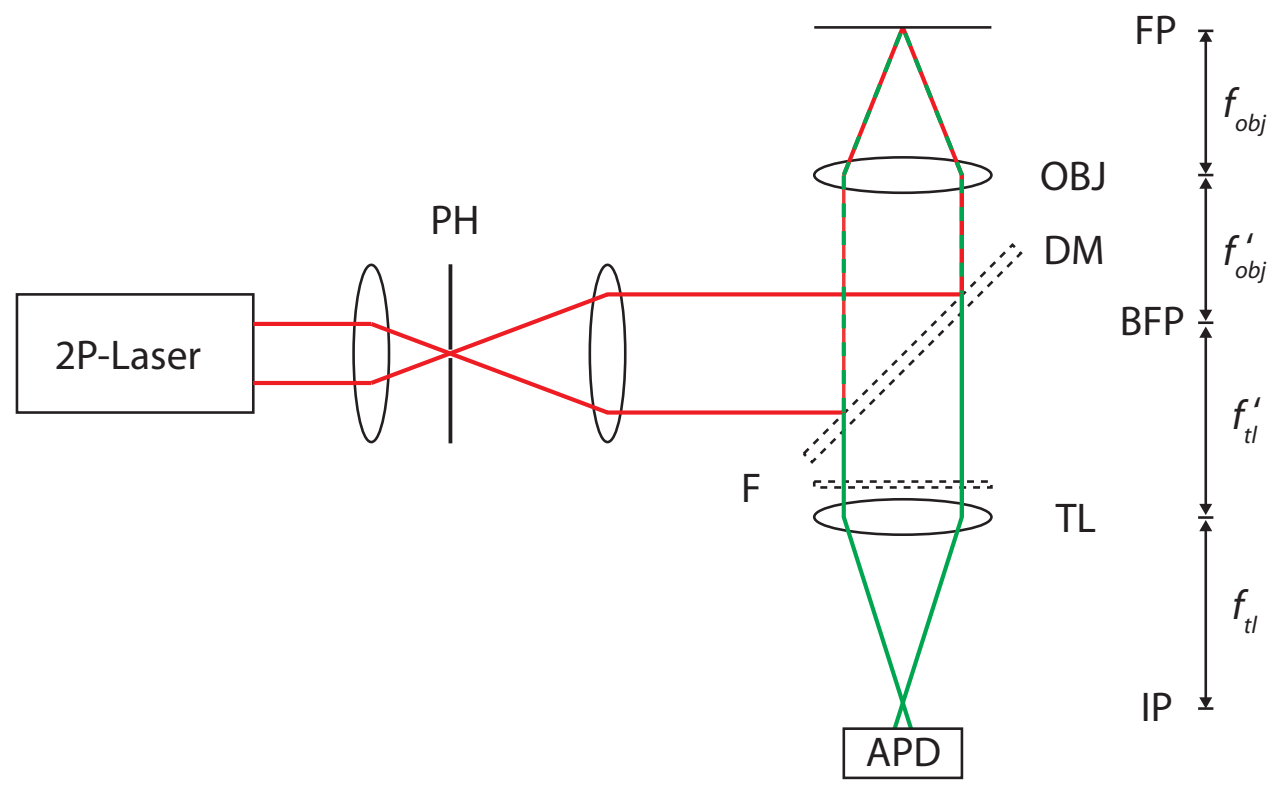

Figure 2.5: Typical 2P microscope setup with a 2P laser source (2P-Laser). The pinhole ( $\mathrm{PH})$ which is illuminated by the laser light (red) serves as a point source and is imaged into the focal plane (FP) of the objective lens (OBJ) with focal length $\mathrm{f}_{\text {obj. }}$. Because of the quadratic intensity dependence of the excitation, the fluorescence generation is confined to the focal volume. The whole fluorescence light (green) is detected by an avalanche photodiode (APD), for example. DM: dichroic mirror, TL: tube lens with focal length $\mathrm{f}_{\mathrm{tl}}$, F: fluorescence filter, BFP : back-focal plane, IP: image plane.

\subsubsection{Two-photon microscopy}

A second way for achieving optical sectioning is to make use of the two-photon (2P) excitation process which was suggested by Maria Göppert-Mayer in 1931 [38] and experimentally shown by Kaiser and Garret in 1963 [39]. The fluorophore is excited by the simultaneous absorption of two photons which have approximately twice the wavelength of the 1P excitation light. Because of the relatively small 2P absorption cross-section, high illumination intensities are necessary which are typically provided by pulsed lasers with high pulse energies. One of the strengths of $2 \mathrm{P}$ microscopy is that the longer illumination wavelengths can penetrate deeper into turbid media because the scattering cross-section strongly decreases for higher wavelengths. Further, the excitation is confined to regions with high intensity. Consequently, only those fluorophores which reside in the focal volume undergo the excitation process. The bleaching process is therefore also effectively confined to this region.

Like the confocal setup in section 2.2.3, the $2 \mathrm{P}$ scanning microscope employs a point source which is imaged into the sample. The fluorescence light is collected by the same lens and separated from the excitation light using a dichroic mirror. Unlike in the confo- 
cal mode, imaging of the fluorescence onto a pinhole is not necessary. This means that not only the complete fluorescence can be detected, but also the system PSF is solely determined by the illumination PSF $h_{\mathrm{ill}}$ [40]. Because of the quadratic intensity dependence of the $2 \mathrm{P}$ excitation, the system PSF $h_{2 \mathrm{P}}(\vec{r})$ is given by the square of the normalized illumination PSF:

$$
h_{2 \mathrm{P}}(\vec{r})=h_{\mathrm{ill}}^{2}(\vec{r}),
$$

whose central xy plane is shown in figure 2.6 (b). Panels (e) and (f) of the same figure present the z-response (cf. equation (2.9)) and the sea-response (cf. equation (2.10)) of the $2 \mathrm{P}$ microscope. It becomes obvious that both the $2 \mathrm{P}$ microscope and the confocal microscope have optical sectioning capabilities. Unlike the confocal mode, which filters the fluorescence light, the optical sectioning in the $2 \mathrm{P}$ mode relies on the confined excitation volume resulting from the non-linear excitation process. Despite the common squaring of the illumination PSFs, the resolution of the $2 \mathrm{P}$ microscope is slightly worse than that of the confocal microscope on account of the longer illumination wavelength. 

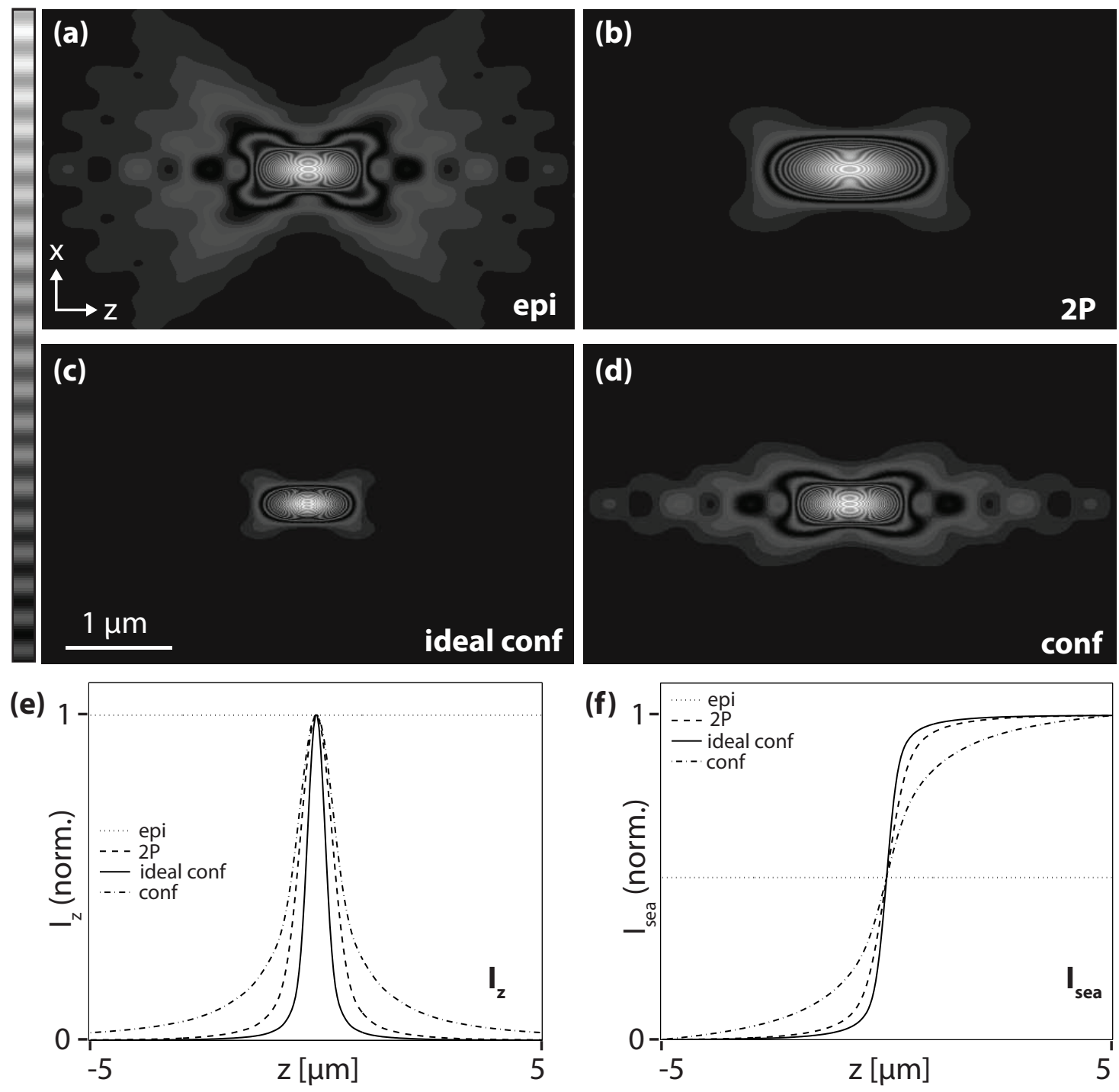

Figure 2.6: Lateral slice through the center of the PSF ( $x, y, z=0)$ of (a) an epifluorescence microscope $\left(\lambda_{\mathrm{det}}=508 \mathrm{~nm}\right)$, (b) a 2P microscope $\left(\lambda_{\mathrm{ill}}=890 \mathrm{~nm}\right)$, (c) an ideal confocal microscope with infinitesimally small pinhole size $\left(\lambda_{\mathrm{ill}}=488 \mathrm{~nm}, \lambda_{\mathrm{det}}=508 \mathrm{~nm}\right),(\mathrm{d})$ a confocal microscope with a pinhole size of the diameter of the Airy disk $\left(\lambda_{\text {ill }}=488 \mathrm{~nm}, \lambda_{\text {det }}=508 \mathrm{~nm}\right)$. (e) Z-response according to equation (2.9) and (f) sea-response according to equation (2.10) for each of the four microscopes. The epifluorescence microscope shows a constant z-response and, therefore, does not have any sectioning capabilities. The ideal confocal PSF features the steepest sea-response. Despite the same quadratic dependance on the illumination PSF, the 2P microscope performs slightly worse because of its longer illumination wavelength. The confocal microscope's PSF with finite pinhole size (one Airy unit) shows slight contributions of out-of-focus planes which broaden the z-response. Nevertheless, the confocal microscope shows distinct sectioning capabilities. 


\section{4Pi multifocal multiphoton microscopy}

As already pointed out in chapter 1, far-field light microscopy allows the carrying out of quantitative studies of biochemical processes in living cells. Thanks to the rapid extension of the GFP-family which enables fluorescent tagging of proteins of interest in living cells, fluorescence microscopy has emerged as an important tool to visualize and understand structure and function in the interior of cells. Prominent examples are confocal microscopy and 2P microscopy with their inherent optical sectioning (cf. section 2.2). Yet, the demand for higher resolution has fueled the development of other microscopy techniques. Among them are those which especially improve the axial resolution like the $\mathrm{I}^{5} \mathrm{M}$ [5], the standing wave microscope (SWM) [41] and the 4Pi microscope which coherently combine the aperture of two objective lenses.

Despite the fact that the first experimental implementation of a 4Pi system already dates 15 years back [42], this technique remains of constant interest. Not only has a commercial system been available since 2005 (TCS 4Pi, Leica Microsystems, Mannheim, Germany), but also recent improvements have been shown with regard to the microscope's transmittance of spatial frequencies. These advancements rely on the introduction of ultra-high aperture objective lenses. Confocal 4Pi microscopy with virtually no side lobes was presented [22] which renders mathematical post-processing obsolete. Moreover, confocal 4Pi microscopy with 1P excitation [23] and the coherent use of both objective lenses for the illumination (4Pi type A) was shown. Here, the typically higher photon flux becomes accessible which can be achieved by linear excitation compared to $2 \mathrm{P}$ excitation. These achievements have only been applied to the imaging of fixed biological samples because ultra-high numerical aperture oil-immersion objective lenses are needed and because of the long image acquisition times in the order of $10 \mathrm{~s}$ for $256 \times 256$ pixels.

When imaging live biological specimens, 4Pi microscopy faces the following difficulties. First, living cells usually necessitate an aqueous embedding medium. This means that the large solid angle which can be used with oil-immersion lenses cannot be fully benefitted from because of total internal reflection at the boundary of the cover slip and the embedding medium. Moreover, a large refractive index mismatch between the immersion medium and the embedding medium leads to spherical aberrations [2]. Consequently, the use of water-immersion objective lenses whose semi-aperture angle is presently limited to $64^{\circ}$ is recommended. Second, imperfect optical conditions which are mainly due to a varying refractive index of the biological structure have to be dealt with. Last, the image acquisition time should be at least on the time-scale of the ongoing dynamics.

The introduction of a beam-scanning confocal MMM 4Pi type A [25] which simulta- 
neously scanned 16-64 foci over the field of view provided fast data acquisition and made the recording of living cells feasible. It was applied to studies of the Golgi apparatus in live mammalian cells [43] [44]. The image acquisition time for $256 \times 256$ pixels was cut down to about $1 \mathrm{~s}$ (plus $1.25 \mathrm{~s}$ for read out of the camera).

\subsection{Fundamentals of 4Pi microscopy}

The resolution of an imaging system scales inversely with its numerical aperture which depends on the used solid aperture angle and the refractive index of the embedding medium (cf. section 2.2). The semi-aperture angle of the objective lens is presently limited by technical issues to about $74^{\circ}$. Likewise, the refractive index cannot be increased arbitrarily because a large refractive index mismatch between the immersion medium and the embedding medium leads to spherical aberrations [2]. The 4Pi microscope doubles the used solid aperture angle by the coherent use of two opposing high-angle objective lenses and hereby mimics the use of an almost full solid angle of $4 \pi$. This approach considerably reduces the PSF's asymmetry along the optic axis. The PSF features a main maximum in the focal plane and primary lobes ("side lobes") of lower intensity which are located about half the wavelength above and below the focal plane. These are accompanied by weaker periodic lobes (cf. figure 3.2 (a)). The main focal maximum of the 4Pi PSF determines the resolution and is narrowed down in the axial direction 3- to 7-fold compared to the confocal PSF. The side lobes lead to ghost images which can be removed mathematically by deconvolution. The quality of the deconvolution depends on the relative height of the side lobes. It is usually unambiguous if the height of the side lobes does not exceed $50 \%$.

Figure 3.1 presents the schematic of a typical 4Pi unit. A collimated excitation beam is split into two beams of equal intensity by a beam splitter. Each of the two beams is imaged onto the back-aperture of one objective lens and focused onto the same point in space. If pulsed excitation is used, the path lengths of both interferometer arms must be equal within the temporal coherence length of the pulses which is typically in the range of $30-60 \mu \mathrm{m}$ for a pulse width of 100-200 fs. This length match is usually achieved by shifting the beam splitter. There are two possibilities for the detection of the fluorescence light. Either the fluorescence light is detected through just one objective lens, separated from the excitation light and imaged onto a detector. This mode is referred to as type A. Alternatively, the fluorescence is collected by both lenses and combined at a second beam splitter where the two parts interfere with each other ("self-interference"). The coherent use of both the illumination and the detection light is termed type C. Here, the short coherence length of the fluorescence light, which is typically in the range of some micrometers, places high demands on the matching of the arm lengths of the second interferometer.

The effective PSF of a confocal 4Pi microscope type A with $2 \mathrm{P}$ excitation is given by

$$
h_{4 \mathrm{Pi}, \mathrm{A}}(\vec{r})=\left(\left|\vec{E}_{1, \mathrm{ill}}+\vec{E}_{2, \mathrm{ill}}\right|^{4}\right) \cdot\left(h_{\mathrm{det}}(\vec{r}) \otimes_{2 \mathrm{D}} p(\vec{r})\right),
$$




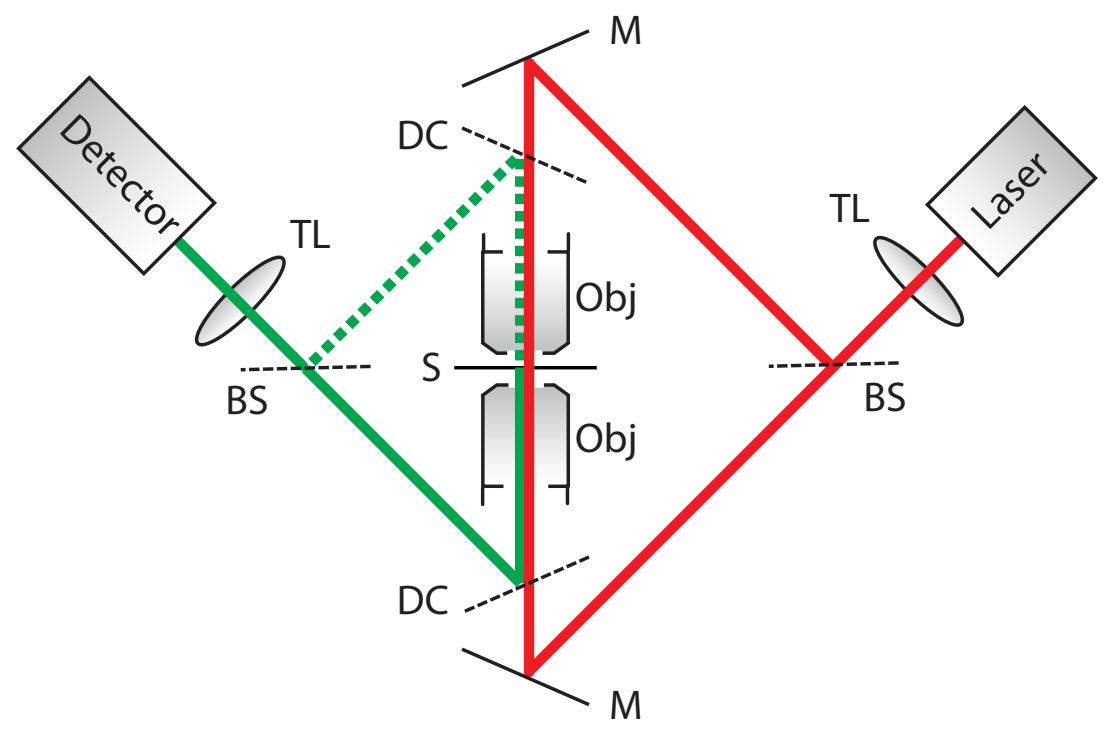

Figure 3.1: Schematic of a 4Pi microscope. The excitation light (red line) is used coherently in type $\mathrm{A}$ and type $\mathrm{C}$ modes. The fluorescence light is only detected through both objective lenses (Obj) in type C (dotted green line). TL: tube lens, BS: beam splitter, M: mirror, DC: dichroic mirror, S: sample.

where the first part represents the excitation PSF and the second part describes the confocal detection. Please refer to section 2.2 for a general introduction to the effective PSFs of imaging systems. $\vec{E}_{1, \text { ill }}$ and $\vec{E}_{2, \text { ill }}=\hat{M} \cdot \vec{E}_{1, \text { ill }}(\hat{M} \cdot \vec{r})$ denote the electric illumination fields generated by both objective lenses under the assumption that the field strengths are equal. The sum of the electric fields is taken to the power of four because of the $2 \mathrm{P}$ absorption probability. $\vec{r}=\vec{r}(x, y, z)$ is the position vector and $\hat{M}$ depends on the interferometric setup and is

$$
\hat{M}:=\left[\begin{array}{rrr}
1 & 0 & 0 \\
0 & 1 & 0 \\
0 & 0 & -1
\end{array}\right]
$$

for a triangular design.

The effective PSF of a confocal 4Pi microscope type $\mathrm{C}$ additionally accounts for the interference of the fluorescence light and is thus given by:

$$
h_{4 \mathrm{Pi}, \mathrm{C}}(\vec{r})=\left(\left|\vec{E}_{1, \mathrm{ill}}+\vec{E}_{2, \mathrm{illl}}\right|^{4}\right) \cdot\left(\left|\vec{E}_{1, \mathrm{det}}+\vec{E}_{2, \mathrm{det}}\right|^{2} \otimes_{2 \mathrm{D}} p(\vec{r})\right),
$$

where $\vec{E}_{1, \text { det }}$ and $\vec{E}_{2, \text { det }}=\hat{M} \cdot \vec{E}_{1, \operatorname{det}}(\hat{M} \cdot \vec{r})$ denote the electric fields of the detection PSF generated by both objective lenses.

A third mode of $4 \mathrm{Pi}$ microscopy is the so-called type B mode which is mainly of scientific interest and which coherently adds the fluorescence, but illuminates only through 
one objective lens:

$$
h_{4 \mathrm{Pi}, \mathrm{B}}(\vec{r})=h_{\mathrm{ill}}^{2} \cdot\left(\left|\vec{E}_{1, \mathrm{det}}+\vec{E}_{2, \mathrm{det}}\right|^{2} \otimes_{2 \mathrm{D}} p(\vec{r})\right) .
$$

The first part represents the 2P excitation PSF and the second part describes the confocal coherent detection.

Because high numerical apertures are used and polarization effects need to be accounted for, the electric fields are usually not described according to (2.1), but with the vectorial diffraction theory [45] [46]:

$$
\vec{E}(\vec{r})=A\left(\begin{array}{c}
i\left(I_{0}(\vec{r})+I_{2}(\vec{r}) \cos (2 \Phi)\right) \\
\left.i I_{2}(\vec{r}) \sin (2 \Phi)\right) \\
2 I_{1}(\vec{r}) \cos (\Phi)
\end{array}\right)
$$

$\Phi$ denotes the azimuth angle of the position vector $\vec{r}$ and the diffraction integrals are given by:

$$
\begin{aligned}
& I_{0}(\vec{r})=\int_{0}^{\alpha} \sqrt{\cos (\theta)} \sin (\theta)(1+\cos (\theta)) J_{0}\left(k \sqrt{x^{2}+y^{2}} \sin (\theta)\right) \exp (i k z \cos (\theta)) d \theta \\
& I_{1}(\vec{r})=\int_{0}^{\alpha} \sqrt{\cos (\theta)} \sin ^{2}(\theta) J_{1}\left(k \sqrt{x^{2}+y^{2}} \sin (\theta)\right) \exp (i k z \cos (\theta)) d \theta \\
& I_{2}(\vec{r})=\int_{0}^{\alpha} \sqrt{\cos (\theta)} \sin (\theta)(1-\cos (\theta)) J_{2}\left(k \sqrt{x^{2}+y^{2}} \sin (\theta)\right) \exp (i k z \cos (\theta)) d \theta,
\end{aligned}
$$

where $J_{0}, J_{1}$ and $J_{2}$ denote the zero, first and second order Bessel functions of first kind.

Figure 3.2 (a) shows the central $x z$ slices $(x, y=0, z)$ of the 4Pi PSFs for the $2 \mathrm{P}$ excitation $\left(\lambda_{2 \mathrm{P}-\mathrm{exc}}=890 \mathrm{~nm}\right)$ and the detection $\left(\lambda_{\mathrm{det}}=504 \mathrm{~nm}\right)$. The corresponding axial profiles along the optic axis illustrate the intensity modulation and show the main lobe and the accompanying weaker lobes. Note that the distance between the primary lobes is approximately the wavelength. The dashed lines depict the axial profiles of the corresponding ideal confocal PSFs. Figure 3.2 (b) compares the effective PSFs of 2P excitation with wide-field detection (top left), the confocal 2P 4Pi type A mode (middle left) and the $2 \mathrm{P}$ 4Pi type $\mathrm{C}$ mode (bottom left). Both effective 4Pi PSFs show a modulation along the axial direction with primary side lobes which are placed at about half the wavelength above and below the focal plane. The $2 \mathrm{P} 4 \mathrm{Pi}$ type $\mathrm{C}$ mode features particularly low primary side lobes which is aided by the fact that the maxima of the excitation PSF fall near the minima of the detection PSF. The ghost images caused by the side lobes can be mathematically eliminated if two necessary conditions are fulfilled. The deconvolution algorithms generally assume that the PSF is space-invariant which means that the phase is constant 

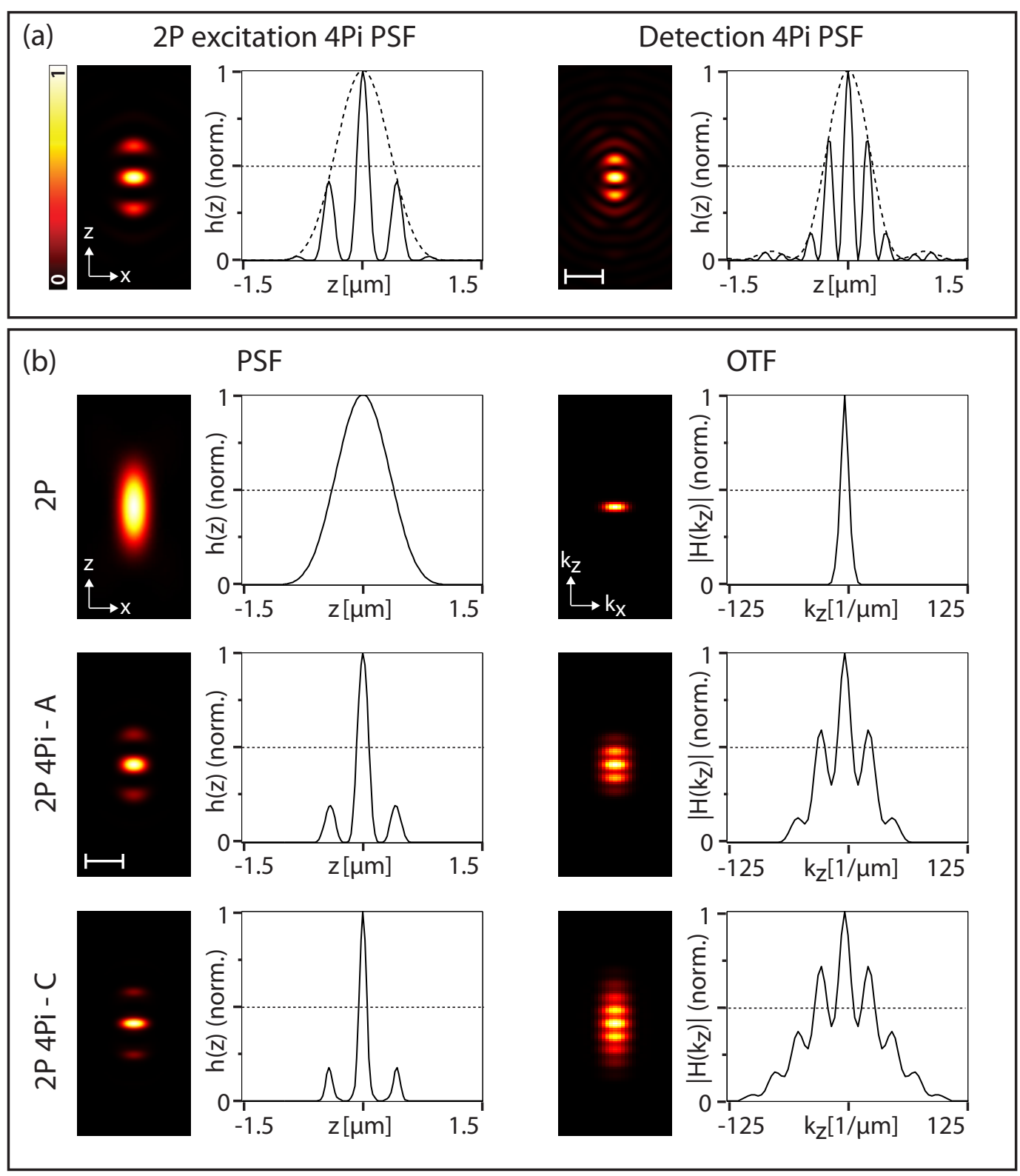

Figure 3.2: (a) Comparison of the intensity 4Pi PSFs for 2P excitation and detection. The central $\mathrm{xz}$ planes and the corresponding axial profiles along the optic axis (black line) are shown. The axial profiles of the ideal confocal PSFs are shown dashed. (b) Comparison of the 2P microscope with wide-field detection (top), the confocal 2P 4Pi type A microscope (middle) and the confocal 2P 4Pi type $\mathrm{C}$ microscope (bottom). The central $\mathrm{xz}$ planes of the PSFs and the corresponding axial profiles along the optic axis (black line) are shown in the left column. The right column depicts the modulus of the corresponding OTFs and the axial profiles through their centers. Parameters: $\lambda_{2 \mathrm{P}-\mathrm{exc}}=890 \mathrm{~nm}, \lambda_{\mathrm{det}}=508 \mathrm{~nm}$, pinhole diameter: 1 Airy unit, refractive index $\mathrm{n}=1.334$, numerical aperture $\mathrm{NA}=1.2$. The scale bar represents $500 \mathrm{~nm}$. 
over the whole field of view. Further, the quality of the deconvolution which ideally results in the elimination of the ghost images depends on the shape of the OTF. If the OTF shows areas with zero values inside its support, the newly gained high frequency parts are disconnected from the low frequencies. In this case, convolution does not work unambiguously [47] and the higher frequencies cannot fully be utilized. As a rule of thumb, the side lobes can be removed reliably if the primary lobe height is less than $50 \%$ of the main lobe. The OTF of the confocal 4Pi PSFs in the right column of figure 3.2 (b) is contiguous and well-filled. Thus, deconvolution algorithms like the fast linear three point (3p) deconvolution [48] or the non-linear Richardson-Lucy deconvolution [49] will work satisfactorily for the $4 \mathrm{Pi}$ data.

From the preceding paragraphs, the options for decreasing the side lobe height can be summarized. First, $2 \mathrm{P}$ excitation lowers the height of the primary lobes due to the quadratic intensity dependence of the absorption cross-section. However, the resolution typically does not benefit from it because of the longer wavelength of the $2 \mathrm{P}$ excitation light. Second, confocalization effectively filters out the primary lobes especially if their positions are far away from the focal plane. Third, the type $\mathrm{C}$ mode makes use of the interference of the excitation and the fluorescence light. The effective lobe height benefits from advantageous distances of the primary lobes of the excitation PSF and of the detection PSF. Here, 2P excitation is again beneficial because the primary lobes are further outside and moreover, they typically fall into the minima of the detection PSF. Two of these options are usually combined in order to obtain a reliable and robust reduction of the side lobes.

\subsubsection{Multifocal illumination in the 4Pi microscope}

A way to speed up the acquisition time is parallelization [34]. Instead of scanning the sample with just a single focus, the beam is split up in several beamlets. This can be done for instance with the help of an array of microlenses or with a microlens disk of Nipkow type in which the microlenses are arranged in an Archimedean spiral. In the former case, the scanning is performed for example by a galvanometric scanner. In the latter case, rotation of the disk leads to homogeneous scanning of the whole field of view. In case of such beam scanning systems, the phase $\Phi$ of the PSF must be constant not only at one particular position, but in the whole field of view. This space invariance is fulfilled if

$$
\left.\Phi_{2}(\hat{M} \cdot \vec{r})\right|_{z=0}=\left.\Phi_{1}(\vec{r})\right|_{z=0}+\text { const }
$$

where $\Phi_{1,2}$ denotes the phase of the electric fields generated by both objective lenses. The phase $\Phi_{1}(x, y, z=0)$ in the focal plane is arbitrary. Equation (3.7) means that the transformation between the planes of equal phase generated by the two objective lenses must be a reflection in the focal plane or in a parallel plane.

$\hat{M}$ is given by definition (3.2) for a triangular implementation. Under the assumption that the field of view is homogeneously scanned during the acquisition of one image, 
(a)

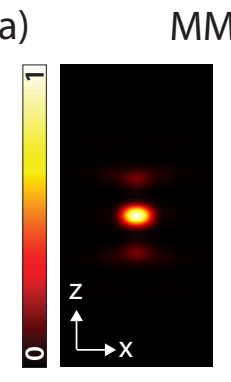

MMM 4Pi type A

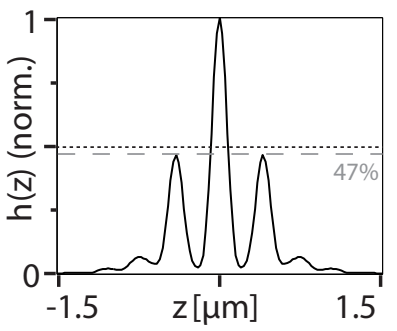

(b)

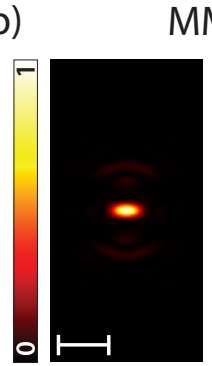

MMM 4Pi type C

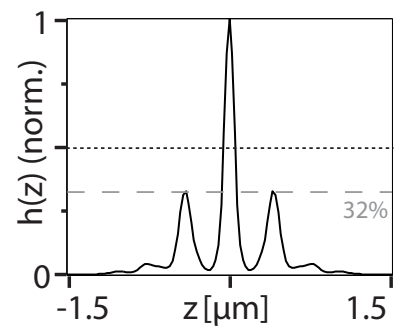

Figure 3.3: Comparison of the non-confocal MMM 4Pi PSFs type A (a) and type C (b). The corresponding axial profiles are integrated over an xy region of $1.5 \times 1.5 \mu \mathrm{m}^{2}$. Parameters: $\lambda_{2 \mathrm{P}-\mathrm{exc}}=890 \mathrm{~nm}, \lambda_{\mathrm{det}}=508 \mathrm{~nm}$, refractive index $\mathrm{n}=1.334$, numerical aperture NA=1.2. The scale bar represents $500 \mathrm{~nm}$.

the illumination PSF of a non-confocal 4Pi type A with 2P excitation and conventional imaging onto a CCD camera is [50]:

$$
h_{\mathrm{MMM}, \mathrm{A}}=\left(\int\left|\left(\vec{E}_{1, \mathrm{ill}}+\vec{E}_{2, \mathrm{ill}}\right) \otimes_{2 \mathrm{D}} g(\vec{r})\right|^{4} d x d y\right) \cdot h_{\mathrm{det}},
$$

where the integral is equivalent to the z-response $I_{z}$ in equation (2.9). $g(\vec{r})$ is the vectorial grid function which describes the coordinates of $\mathrm{N}$ foci in the focal plane:

$$
\vec{g}(\vec{r})=\left(\begin{array}{l}
1 \\
1 \\
1
\end{array}\right) \cdot \sum_{n=1}^{N} \delta\left(x-x_{n}\right) \delta\left(y-y_{n}\right) \delta(z),
$$

where $\delta$ is Dirac's delta function. The lateral resolution is determined by the second part of equation (3.8) which represents the PSF of a conventional epifluorescence microscope.

The corresponding PSF for type $\mathrm{C}$ is:

$$
h_{\mathrm{MMM}, \mathrm{C}}=\left(\int\left|\left(\vec{E}_{1, \mathrm{ill}}+\vec{E}_{2, \mathrm{ill}}\right) \otimes_{2 \mathrm{D}} \vec{g}(\vec{r})\right|^{4} d x d y\right) \cdot\left(\left|\vec{E}_{1, \mathrm{det}}+\vec{E}_{2, \mathrm{det}}\right|^{2} \otimes_{2 \mathrm{D}} \vec{g}(\vec{r})\right) .
$$

Figure 3.3 compares the PSFs of the non-confocal MMM 4Pi type A mode and the type $\mathrm{C}$ mode. It is assumed that the grating is sufficiently sparse so that the interference of adjacent foci is negligible. The vectorial grid function $\vec{g}(\vec{r})$ can therefore be neglected. The axial profiles integrated over an xy region of $1.5 \times 1.5 \mu \mathrm{m}^{2}$ reveal that the combination of coherent illumination and detection decreases the relative side lobe height by approximately $15 \%$ compared to the coherent use of both objective lenses for only the illumination.

Parallelization is particularly interesting if the $2 \mathrm{P}$ laser system offers so much power that laser light needs to be dumped in order not to deposit too much energy in one single 
focus. The limit on the peak intensity for biological systems is considered to be approximately $200 \mathrm{GW} / \mathrm{cm}^{2}$ using $200 \mathrm{fs}$ pulses [51]. The excess power can therefore be used for the other foci.

An issue of parallelization is that the illumination of a periodic microlens array or disk results in repeated self-imaging of the foci in characteristic planes which are perpendicular to the optic axis. The sample might be effectively excited in these so-called (fractional) Talbot planes [52] which can increase the background signal considerably.

\subsubsection{Confocalization by post-processing}

The optical sectioning effect, which can be achieved by placing a confocal pinhole in the detection path, can also be attained by a modified illumination scheme in conjunction with post-processing [53]. Instead of homogeneously illuminating the field of view, the illumination is structured, for example, in a point or grid pattern. At a given axial position of the sample, a set of images is acquired, each recorded at a different position of the illumination pattern. Methods for deriving an optically sectioned image from such a set of images typically estimate the local degree of intensity modulation [54]. Those parts of the sample which are near the focal plane are excited by a strongly modulated intensity. The fluorescence emitted from the same volume is therefore also strongly intensity-modulated. On the other hand, those parts of the sample which are out of focus are more homogeneously illuminated. Further, the fluorescence emitted from these regions is blurred on the detector which sits in the conjugated image plane. Consequently, the signal which stems from the out-of-focus regions shows weaker modulation upon variation of the lateral position of the illumination pattern.

Computational analysis can discriminate the strongly modulated signal from the weakly modulated background signal which is tantamount to confocalization. Consequently, optical sectioning is rendered possible without introducing real pinholes into the detection path. Computational confocalization provides more flexibility because the trade-off between the signal and the sectioning strength can be dealt with after the data recording. On the other hand, a set of images at every axial position is needed instead of just a single frame. This means that one has to handle a large data throughput. Further, the signalto-noise ratio (SNR) is decreased for a given acquisition time because of the patterned illumination instead of a wide-field illumination. In order to maintain a given SNR, the data acquisition time can be increased provided that the fluorophores are sufficiently stable.

If the spacing of the repetitive patterns (points or lines) in the illumination is much larger than the width of the detection PSF, the fast 'superconfocal' method is applicable and provides good optical sectioning capabilities [55]. The reconstructed slice $\mathrm{I}_{\mathrm{rec}}$ is given by

$$
I_{\mathrm{rec}}=\max _{n=1 \ldots N}\left[I_{n}(x, y)\right]+\min _{n=1 \ldots N}\left[I_{n}(x, y)\right]-2\left(\frac{1}{N} \sum_{n=1 \ldots N} I_{n}(x, y)\right)
$$


where $I_{n}$ denotes the pixel intensity and $N$ denotes the total number of pixels. The maximum of each pixel contains mainly information from the focal plane and the background, whereas the minimum of each pixel is mainly comprised of contributions from out-offocus planes. The subtraction of twice the average image in the last part of equation (3.11) relies on the assumption that the in-focus information is only illuminated for some positions of the illumination pattern which leads to a distorted histogram of detected intensities.

The reconstruction of the data is non-linear which means that the final image $I_{\text {rec }}$ cannot be described by the convolution of the spatial distribution of fluorescence emitters and the PSF. This can lead to changes of the apparent size and shape of objects and of their integrated intensity which must be considered when performing a quantitative analysis.

\subsection{Experimental implementation}

Motivated by the findings of the preceding paragraphs, a new MMM 4Pi type C setup was realized with $2 \mathrm{P}$ excitation so as to effectively lower the relative side lobe height. As an alternative for reducing the lobe height, MMM 4Pi type A in combination with post-processing-confocalization was harnessed. Since imaging of living cells requires an aqueous embedding medium, water-immersion objective lenses were used.

A spinning microlens disk was used for increasing the frame acquisition rate up to the limit which is imposed by the photon flux and the SNR, respectively. Moreover, cameras which are capable of simultaneous image acquisition and read out were used. This avoids any dead times during the measurement protocol. It is challenging to combine the concepts of confocal detection and scanning by rotation of microlenses. Either a synchronization of the microlenses and the pinhole array which sits in an image plane is necessary or the lateral movement of the foci must be descanned in front of the pinhole array. The latter requires additional optics in the detection path. Therefore, no detection pinholes were used which entails the advantage that no fluorescence signal is lost at the pinholes. Further, the coherent use of both objective lenses for the detection typically doubled the fluorescence signal.

\section{Setup and basic alignment routines}

Figure 3.4 illustrates the main components of the implementation of the MMM 4Pi microscope type A (panel (a)) and type C (panel (b)). The setup is described in more detail in the following.

The 2P excitation source was a mode-locked Titanium:Sapphire-laser (Ti:Sa-laser) delivering $140 \mathrm{fs}$ pulses at a wavelength between $690 \mathrm{~nm}$ and $1040 \mathrm{~nm}$ at a repetition rate of $80 \mathrm{MHz}$ (Chameleon Ultra, Coherent Inc., Santa Clara, Ca, USA). For protection of the light source, a Faraday rotator (FL500/-1100-8Sl, LINOS Photonics GmbH \& Co. KG, Göttingen, Germany) was placed in front of the Ti:Sa-laser. The laser intensity which is delivered to the 4Pi cavity was controlled by a laser power controller (LPC, Brockton 

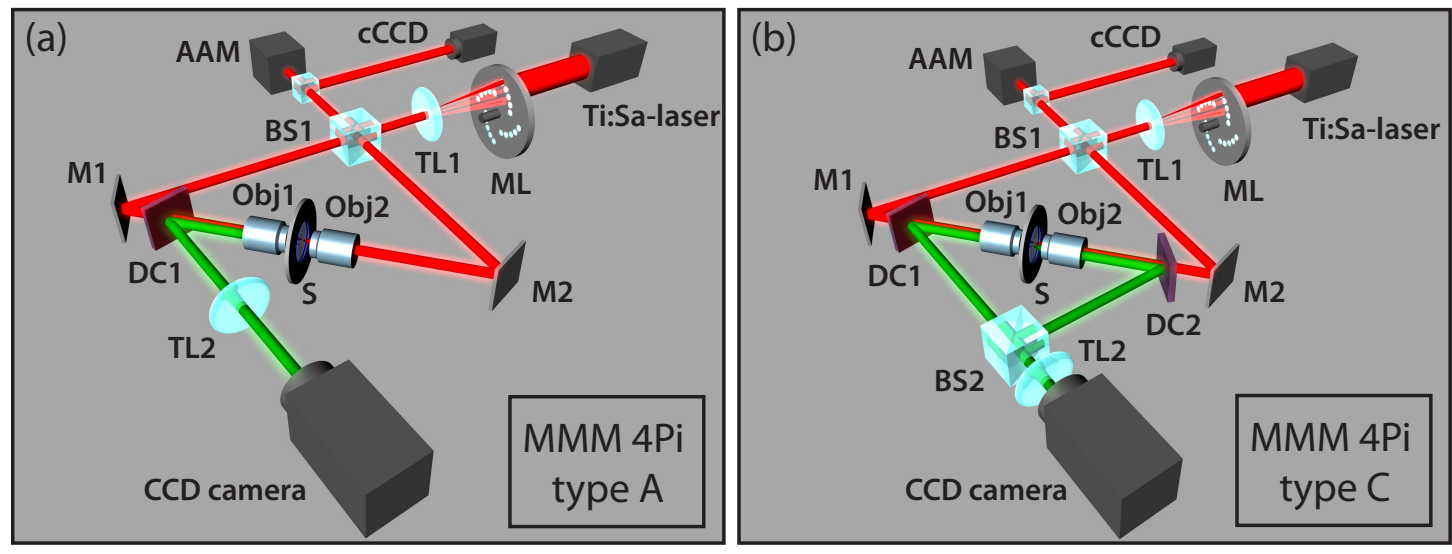

Figure 3.4: Schematics of the main components of the MMM 4Pi setup (a) in type A configuration and (b) in type $\mathrm{C}$ configuration. The $2 \mathrm{P}$ excitation beam (red line), which is delivered by the mode-locked Ti:Sa-laser is split up in several beamlets by a disk of microlenses (ML) and subsequently coupled into the illumination cavity by a beam splitter (BS1). The tube lens (TL1) images the microlenses into the back-focal plane of the objective lenses (Obj1, Obj2). The sample (S) is illuminated coherently through both lenses. The fluorescence (green line) is separated from the excitation light by a dichroic long-pass filter (DC1, DC2). In type A configuration (left panel), it is detected through only one objective (Obj1) and imaged onto the detector (CCD camera) by the detection tube lens (TL2). In type C configuration (right panel), the fluorescence, which is detected through both objective lenses, is combined at the detection beam splitter before being imaged onto the detector. The alignment of the illumination cavity is facilitated by a control camera (cCCD). The alignment of the objective lenses can be automatically monitored and controlled by the automatic alignment module (AAM). M1, M2: mirror.

Electro-Optics Corp., Bridgewater, MA, USA). The beam was expanded and collimated by a beam expander (S6ASS2075/-94, Sill Optics GmbH \& Co. KG, Wendelstein, Germany). It was then split into 15-20 beamlets by a microlens disk with 5624 microlenses (each of diameter: $512 \mu \mathrm{m}$, focal length: $12 \mathrm{~mm}$ ). The disk was comprised of eight segments (only four are shown in figure 3.4 for clarity), in which the lenses are arranged as a helix (Nipkow type), such that one rotation of the disk led to an 8-fold homogeneous lateral scanning of the field of view. The disk was driven by a servo motor (Series 3565K, Faulhaber GmbH \& Co.KG, Schönaich, Germany). The beamlets were subsequently coupled into the 4Pi cavity which was aligned vertical to the optical table. This entailed the advantage that the sample is oriented horizontally which simplifies its mounting and allows the use of dipping lenses. Note that figure 3.4 shows a horizontal alignment for simplicity. The beam diameter was adjusted by three achromatic lenses such that the back-aperture of the objective lens was over-illuminated. Subsequently, the beam was split into the two arms of the illumination interferometer by a beam splitter.

The water-immersion objective lenses (HCX PL APO 63X, 1.20 W CORR CS, Leica Microsystems, Wetzlar, Germany) opposed each other and shared the same optic axis. They were aligned such that their excitation foci overlapped in all three dimensions. The objec- 
tive lenses were equipped with a correction ring which was used for adjusting the wavefronts to the thickness of the cover slip. This was necessary because the refractive index mismatch of the immersion medium $(n=1.33)$ and the borosilicate cover slip $(n=1.52)$ induced spherical aberrations which needed to be compensated by pre-aberrating. The longitudinal chromatic aberrations of the two objective lenses were such that the aberrations for $890 \mathrm{~nm}$ were similar to the aberrations for the fluorescence wavelength $(\approx 500 \mathrm{~nm})$. Further, the longitudinal chromatic aberration behavior of the objective lenses had to be matched so that the difference of the focal lengths for the excitation wavelength and for the fluorescence wavelength were equal for both objective lenses. If this is not the case, the alignment of the detection cavity is hampered. This is because the image planes of both objective lenses do not coincide so that the images of the fluorescence foci are not in focus for each of the lenses.

The alignment of the 4Pi cavity involved several steps including overlapping the excitation foci, making the plane of equal phase as well as the cover slips perpendicular to the optic axis, matching the arm lengths and shifting the phase, which called for sophisticated alignment options of particular parts of the setup.

The sample was supported in three points by piezo-electric actuators (PA65-14SG, Piezo-system Jena GmbH, Jena, Germany) which could be controlled individually as well as collectively. Their main purpose was to precisely scan the sample along the axial direction within their travel range of $75 \mu \mathrm{m}$ (closed-loop). Additionally, the sample position could be coarsely adjusted in all three dimensions by piezo actuators (Picomotor model 8321, New Focus, San Jose, USA).

The lower objective lens (Obj2) position could be coarsely adjusted by a modified multi-axis-positioner (LP-1A Series, Newport Spectra-Physics GmbH, Darmstadt, Germany) and could be locked. The upper objective lens (Obj1) was supported more flexibly and could be not only coarsely adjusted by micrometer screws, but also precisely controlled by a piezo-electric high precision scanner (P-733K034, Physik Instrumente GmbH \& Co. KG, Karlsruhe, Germany) in the lateral direction and by a piezo actuator (MIPOS 100 PL, Piezosystem GmbH, Jena, Germany) in the axial direction. The relative positions of the two objective lenses could be monitored and adjusted with a control software ${ }^{1}$.

The lengths of the two interferometer arms were matched by shifting the beam splitter (BS1) along the optic axis of one arm. This was simplified by a precise piezo actuator $(P$ 841.20, Physik Instrumente GmbH \& Co. KG, Karlsruhe, Germany) with a travel range of $30 \mu \mathrm{m}$, which was also used for adjusting the relative phase of the interference pattern.

As mentioned above, it is important that the excitation PSF is invariant over the whole

1 Automatic alignment module (AAM): An additional infrared laser beam of low power was sent into the illumination interferometer into both directions via the unused side of the beam splitter. The beams were coupled out in the same way and they were imaged onto a photodiode which sat in the back-projected image plane. The photodiode saw the two images of the beams in the focal plane of the objective lenses. If the objective lenses' foci do not overlap, their images move apart (laterally) or are blurred (axially) and the photodiode detects less signal. A software controled the piezo-electric actuators at the upper objective lens and maximized the photodiode signal by iteratively moving the position of the objective lens in all spatial directions. 
field of view meaning that its phase is constant. If the plane of equal phase does not coincide with the focal plane, but is tilted with respect to it, this can be corrected by shifting the laser beam which enters the objective lens. In doing so, the positions of the foci remain constant. In practice, the upper mirror (M1) of the interferometer was slightly tilted which resulted in the desired shift of the beam and in a concomitant change of the angle between the beam and the optic axis. This angular offset shifted the foci in the lateral direction. However, because of the large distance between the mirror and the lens compared to the focal length, this shift could be compensated by a lateral adjustment of the objective lens (Obj1). The resulting change of phase was much smaller than the first one so that an iteration of the two steps quickly converged. This iterative process changed the length of the upper interferometer arm slightly. However, this was usually negligible because of the comparatively long coherence length of the $2 \mathrm{P}$ excitation light $(\approx 42 \mu \mathrm{m})$. The upper mirror was equipped with piezo-electric actuators (P853.00, Physik Instrumente (PI) GmbH \& Co. KG, Karlsruhe/Palmbach, Germany).

The detection cavity was built analogous to the illumination cavity, but it was mirrored at the optic axis. The fluorescence was separated from the excitation light by two dichroic mirrors which served as the upper and lower mirror of the cavity (650 DCXR, AHF Analysentechnik $A G$, Tübingen, Germany). The two beams could be overlapped at the beam splitter (BS2) by tilting the lower dichroic mirror (DC2).

The lengths of the two arms were matched by shifting the beam splitter (BS2) along the optic axis of one arm. A piezo actuator was used for precise positioning (P853.00, Physik Instrumente (PI) GmbH \& Co. KG, Karlsruhe/Palmbach, Germany) over a range of $30 \mu \mathrm{m}$. A picomotor (8351 Tiny, New Focus, San Jose, USA) was utilized for coarse alignment over a range of several millimeters. The best possible modulation of the interference is only achieved when the optical path lengths match within the center region of the coherence length of the fluorescence.

The planes of equal phase were tilted into the horizontal plane as described above. Here, the iterative process was performed by tilting the beam splitter (BS2) and the lower dichroic mirror (DC2). This process changed the length of one arm slightly. Because of the comparatively short coherence length of the fluorescence, the arm lengths of the detection cavity had to be readjusted.

The fluorescence was spectrally filtered by a short-pass filter (E 720 SP, AHF Analysentechnik AG, Tübingen, Germany) and a band-pass filter (HQ 532/70, AHF Analysentechnik $A G$, Tübingen, Germany). Subsequently, it was imaged onto a CCD camera chip by the tube lens (TL2). Two different cameras were available. The first camera was an interline ${ }^{2}$ CCD camera (Imager Intense, La Vision GmbH, Göttingen, Germany) with 1376x1040 pixels. The chip had a peak quantum efficiency of $65 \%$ at $500 \mathrm{~nm}$ and the readout noise was less than five electrons per pixel. The chip was operated with $2 \times 2$ binning which allowed a maximum frame rate of $50 \mathrm{~Hz}$. The effective pixel size $(116 \mathrm{~nm})$,

$2 \quad$ Each line of photo-sensitive pixels is neighbored by a line of masked storage pixels. Thus, read out and detection can be done simultaneously. However, the active area is considerably reduced by the storage region. 
which is the size of the pixel when it is projected onto the focal plane of the objective lens, was chosen according to the Nyquist-Shannon criterion [37]. This requires a sampling at least twice as fine as the width of the detection PSF in order not to lose any information. The effective size of the chip was $80 \times 60 \mu \mathrm{m}^{2}$.

The second camera was a frame-transfer ${ }^{3}$ electron-multiplying CCD (EMCCD) chip (Ixon plus DU-860, Andor Technology, Belfast, Northern Ireland) with 128x128 pixels. Because the chip was back-illuminated, a high peak quantum efficiency of $92 \%$ (at $575 \mathrm{~nm}$ ) was achieved. The maximum frame rate was $500 \mathrm{~Hz}$ with a duty cycle of $97 \%$. This chip had a multiplication register in which the electrons were amplified by impact ionization prior to read out. Because the readout noise was not amplified, it became negligible (effectively less than one electron per pixel) at high multiplication factors. The effective pixel size was $103 \mathrm{~nm}$. The full chip had therefore an effective size of 13.2x13.2 $\mu \mathrm{m}^{2}$.

Switching from the 4Pi type $\mathrm{C}$ imaging mode to the 4Pi type A mode was simply done by blocking the fluorescence light in one arm of the detection cavity.

The integration of a wide-field microscope into the setup considerably simplified the examination of samples because of its large field of view and a negligible alignment effort. Illumination light from an external light source (EL6000, Leica Microsystems, Wetzlar, Germany) was spectrally filtered by a filter cube (I3, Leica Microsystems, Wetzlar, Germany) and coupled into the upper objective lens by a removable mirror which was placed between one objective lens and the corresponding dichroic mirror. The fluorescence was transmitted by the filter cube and imaged onto a CMOS camera (complementary metal oxide semiconductor camera) (PCVC 840K/20, Philips GmbH, Hamburg, Germany).

\section{Active phase compensation}

As mentioned above, the spatial invariance of the PSF in the focal plane has to be ensured. If the refractive index of the embedding medium is not matched to the one of the immersion medium, the optical path length between the objective lens and its focal plane changes during axial scanning of the sample. This means that the relative phase shift in the focal plane is proportional to the axial displacement of the sample [56]. This was actively compensated by translating the beam splitter along the optic axis of one interferometer arm proportionally to the axial movement of the sample. Hereby, the optical path length was kept constant. If both objective lenses are coherently used for only the illumination, the phase compensation is only necessary in the illumination interferometer. If the 4Pi type $\mathrm{C}$ mode is used, the active phase compensation has to be introduced into the detection interferometer as well.

3 The full frame can be shifted to a masked storage area of equal size, from where the electrons are read out. Hereby, simultaneous detection and read out is possible. 


\subsection{Experimental results for two recording schemes}

In this section, the measurements in two different recording schemes will be presented. Fluorescent beads and GFP-tagged mitochondria in budding yeast (Saccharomyces cerevisiae) were used as samples. Mitochondria are essential organelles of eukaryotic cells. Their prominent function is to supply the cell with energy from oxidative phosphorylation. In budding yeast, these organelles form a highly dynamic branched tubular network with a diameter of about $350 \mathrm{~nm}$ [25]. Under conditions in which the cell adapts to a change of the carbon source, the frequency of balanced matrix separation and fission events is two per minute [57]. It is therefore of great interest to access the 3D structure of live mitochondria at high resolution and on a time-scale of $30 \mathrm{~s}$.

The following parameters apply for all measurements presented in this section. The excitation wavelength was $890 \mathrm{~nm}$ which represents a trade-off between the wavelengthdependent $2 \mathrm{P}$ absorption cross-section of eGFP [58] and the available wavelength-dependent laser power. The time-averaged laser power was approximately $1 \mathrm{~W}$ at $890 \mathrm{~nm}$. Because of power losses at the intermediate optics, only $25 \mathrm{~mW}$ were available for exciting the sample in a field of view with a diameter of $20 \mu \mathrm{m}$. Since the laser beam was split into 19 beamlets, the time-averaged power per focal region was $1.3 \mathrm{~mW}$. This corresponds to a focal peak intensity of $\approx 90 \mathrm{GW} / \mathrm{cm}^{2}$ which is well below $200 \mathrm{GW} / \mathrm{cm}^{2}$. This value is considered as an upper limit for biological samples in non-linear microscopy when using $200 \mathrm{fs}$ pulses [51]. It can be taken as a reference for the pulses of the Ti:Sa laser system used in this experiment, since its pulses were broadened by dispersion in the optics between the laser and the sample. The axial scan of the sample was done in steps of $50 \mathrm{~nm}$.

\section{Preparation of samples}

Because the samples were illuminated (and imaged) through two objective lenses, the specimens had to be placed between two cover slips. The cover slips' thicknesses strongly influence the spherical aberrations induced by the refractive index mismatch between the water-immersion and the cover slip. This was compensated by adjusting the correction rings of the objective lenses to the thicknesses of the cover slips. In order to prevent that this time-consuming adjustment had to be done for every sample, the cover slips were pre-selected for their thickness with a precision of $\pm 1 \mu \mathrm{m}$. Usually, a fraction of the surface of one of the two cover slips used for mounting the sample was covered by a reflective coating. This facilitated the adjustment of the plane of equal phase of the illumination light. Likewise, axial positioning of the sample was simplified. Further, one cover slip was covered by a homogeneous layer of fluorescent beads (FluoSpheres carboxylatemodified microspheres, $0.1 \mu \mathrm{m}$, Invitrogen, Carlsbad, CA, USA). This thin layer acted as a reference layer for the adjustment of the phase of the coherently combined fluorescence. Generally, fluorescent beads are attached to the cover slip by the following procedure. The 

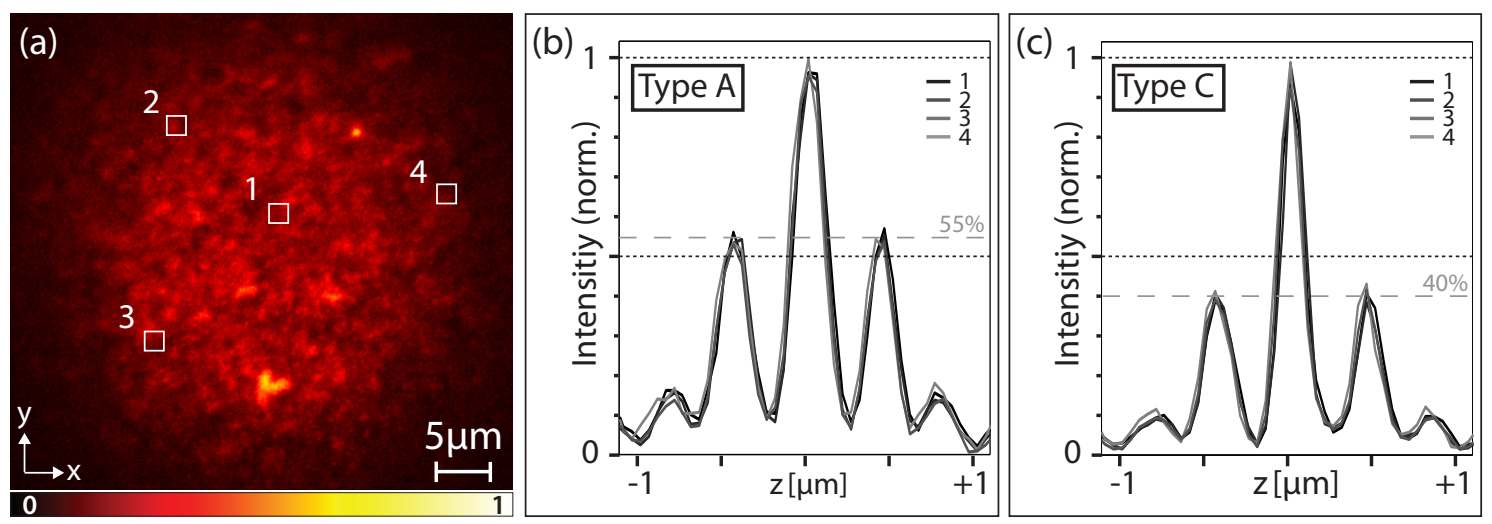

Figure 3.5: A layer of $100 \mathrm{~nm}$ fluorescent beads was recorded in MMM 4Pi type A and type C mode. (a) Xy image at $z=0$. The field of view is only restricted by the size of the laser beam. (b, c) The $z$ profiles of four random regions (marked by the white squares in (a)) show the translationinvariance of the PSF. The average side lobe height is ca. $55 \%$ in type A mode (b) and ca. $40 \%$ in type $\mathrm{C}$ mode (c).

glass surface is incubated with poly-L-lysine (Sigma-Aldrich Chemie GmbH, Taufkirchen, Germany) for five minutes. After removal of the substance, a positively charged layer remains on the glass surface. The surface is then incubated by a diluted solution of the beads for five minutes. Some beads adhere to the surface whose concentration depend on the dilution and the incubation time.

In addition to the technical samples, GFP-tagged mitochondrial network in fixed and living budding yeast were studied. Please refer to the appendix for details on cell cultivation. For imaging purposes, after a washing step, the cells were embedded in $\mathrm{SD}^{4}$ medium with $1 \%$ agarose and mounted between two coverslips. The agarose was added to avoid spatial movement of the yeast cells. The refractive index of the agarose-medium had been adjusted to 1.34 to match the refractive index of the budding yeast.

\section{Results for the MMM 4Pi type C mode}

For the MMM 4Pi measurements in type $\mathrm{C}$ mode, the rotation speed of the microlens disk was set to 3,000 rpm which means that the full field of view was scanned at a frequency of $400 \mathrm{~Hz}$. The exposure time of the Imager-camera was $40 \mathrm{~ms}$ which was only limited by the photon flux and not by the scanning system. The field of view was therefore scanned 16-fold during the acquisition of one image.

A layer of $100 \mathrm{~nm}$ fluorescent beads was imaged first in type A and then in type C mode. The field of view in figure 3.5 (a) was only restricted by the diameter of the excitation beam. The area in which the fluorescence intensity was above $25 \%$ of the maximum intensity had a diameter of approximately $20 \mu \mathrm{m}$. This diameter corresponds to the FWHM of the laser beam because of the quadratic intensity dependence of the $2 \mathrm{P}$

$4 \quad$ SD medium: $74 \%$ glucose, $18.5 \%\left(\mathrm{NH}_{4}\right) 2 \mathrm{SO}_{4}, 6.3 \%$ yeast nitrogen base, $1.2 \%$ amino acids 


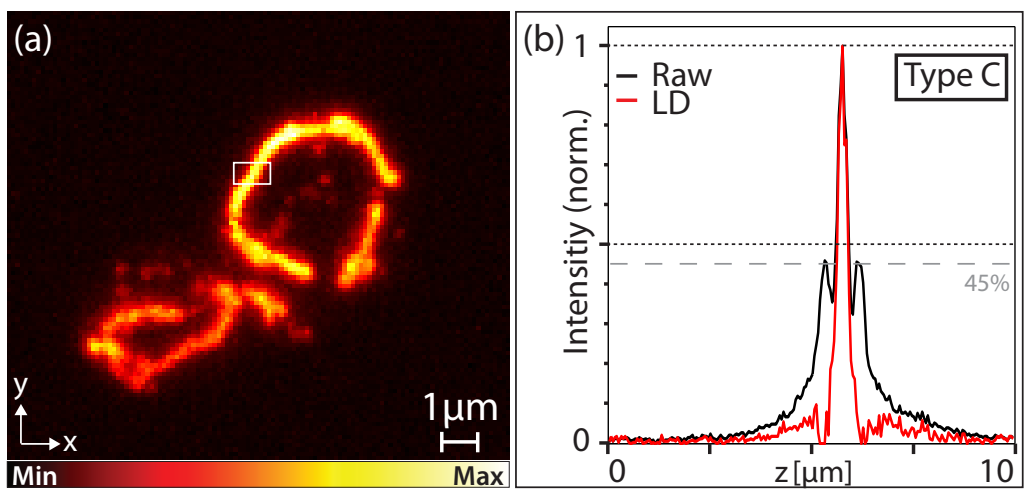

Figure 3.6: MMM 4Pi type $\mathrm{C}$ measurement of live budding yeast. (a) shows the maximum projection of $200 \mathrm{xy}$ images and (b) presents the axial profile, which is integrated over the region marked by the white rectangle in (a), of the raw data (black) and the linearly deconvolved (LD) data (red).

absorption. The averaged $\mathrm{z}$ profiles of four random regions across the field of view are shown in figure 3.5 (b) for the MMM 4Pi type A measurements and in figure 3.5 (c) for the MMM 4Pi type $\mathrm{C}$ measurements. The respective profiles overlap nicely which proves that the PSF is translation-invariant over the field of view. The average side lobe height of the MMM 4Pi type A measurement is $55 \%$ of the main lobe and $40 \%$ for the MMM 4Pi type $\mathrm{C}$ measurement. The comparison of these values to the theoretical results in figure 3.3 show that the improvement of the lobe height is in the expected range, although the absolute lobe height is approximately $7 \%$ above the optimum value. This is probably due to spherical aberrations caused by the optics in the illumination path. The linear deconvolution of the interpolated bead layer with a $100 \mathrm{~nm}$ bead reveals that the axial resolution is at least $140 \mathrm{~nm}$ in the MMM 4Pi type C mode. Since it is not known whether the bead layer is a monolayer or not, this value is a conservative estimate of the axial resolution.

The feasibility of the MMM 4Pi type $\mathrm{C}$ mode for studies of live biological samples was shown by the measurement of a live budding yeast cell. The maximum projection of a stack of 200 xy slices with an axial distance of $50 \mathrm{~nm}$ is shown in figure 3.6 (a). The recording time of the whole stack was $15.6 \mathrm{~s}$ of which approximately half is attributed to the time needed for axial scanning of the sample. Figure 3.6 (b) shows the axial profile of the region marked in (a) by the white rectangle. The raw data is shown in black and the linearly five point (5p) deconvolved data in red. The side lobe height is slightly increased compared to the layer of beads in figure 3.5 probably due to refractive index inhomogeneities in the sample. Nevertheless, the lobe height is well below $50 \%$ which means that the data can be unambiguously deconvolved. 

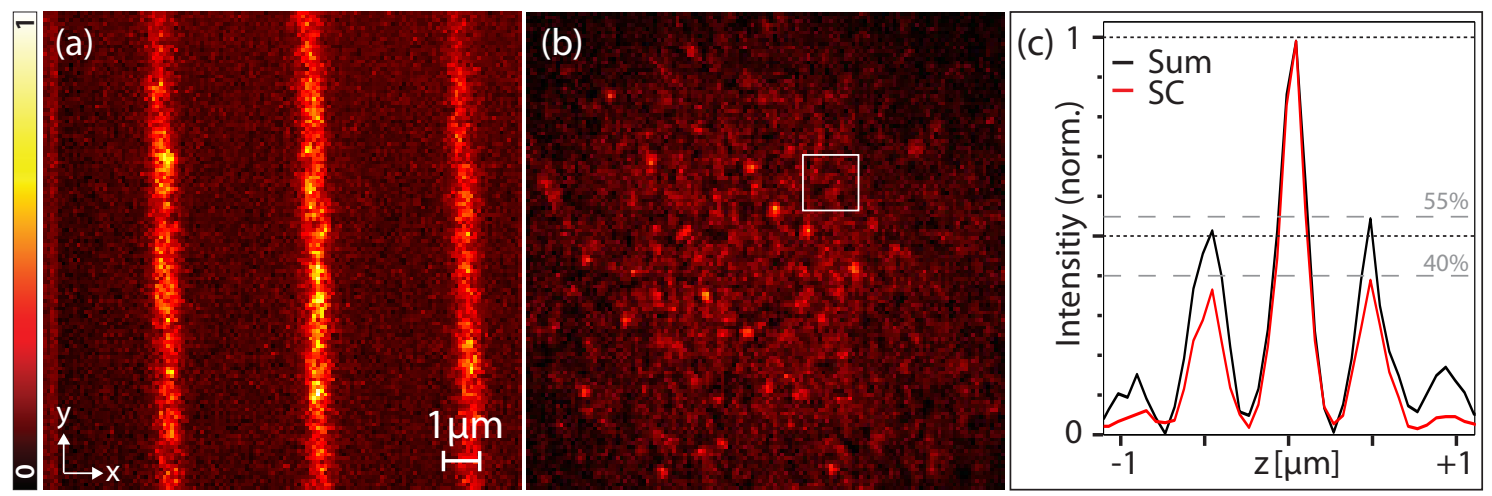

Figure 3.7: MMM 4Pi measurement in type A with post-processing-confocalization of a fluorescent bead layer. (a) The frame recorded with an exposure time of $2 \mathrm{~ms}$ shows the stripe pattern in the illumination. (b) The reconstructed xy image at $\mathrm{z}=0$ after post-processing with the 'superconfocal' (SC) algorithm. (c) The z profiles of the region marked in panel (b) show that the post-processing reduces the side lobe height by ca. $15 \%$ (red line) compared to the axial profile of the sum of every set of frames (black line).

\section{Results for MMM 4Pi type A mode with post-processing-confocalization}

Since the MMM 4Pi type A mode features primary lobe heights of above $50 \%$ (cf. figure 3.5), the recorded data cannot be unambiguously deconvolved. However, MMM 4Pi type $\mathrm{A}$ in conjunction with post-processing-confocalization (see section 3.1.2) turns out to significantly reduce the side lobe heights.

The Ixon-camera was used for the MMM 4Pi type A measurements in conjunction with post-processing-confocalization. Because of its high frame rate (cf. section 3.2), the recording of a set of frames took only twice as long as the recording of one frame at a constant axial position in the type $\mathrm{C}$ mode. Further, its negligible readout noise did not compromise the SNR.

The rotation speed of the microlens disk was $380 \mathrm{rpm}$ which means that the field of view was fully scanned within $20 \mathrm{~ms}$. The exposure time of the Ixon-camera was $2 \mathrm{~ms}$ meaning that only a tenth of the field of view was scanned during the acquisition of one frame. Figure 3.7 (a) illustrates the resulting stripe pattern. The stripes are separated by $4 \mu \mathrm{m}$ and they are $0.5 \mu \mathrm{m}$ wide. Every $2 \mathrm{~ms}$, the pattern moved by ca. $0.4 \mu \mathrm{m}$ in the $\mathrm{x}$ direction. A set of 40 images was taken at each axial position. The total acquisition time of $80 \mathrm{~ms}$ is twice the frame acquisition time of the MMM 4Pi type $\mathrm{C}$ measurements. The sum of all xy images does not show any modulation of the intensity. Figure 3.7 (b) illustrates the xy image which was reconstructed from all sets of images with the help of the 'superconfocal'-algorithm (cf. section 3.1.2). Panel (c) depicts the averaged axial profile of the marked region in (b). The red line shows the axial profile after application of the algorithm, whereas the black line is obtained from the sum of each set of images. The comparison of the two profiles reveals the optical sectioning effect of the 'superconfocal'algorithm which reduces the side lobe height from ca. $53 \%$ to ca. $38 \%$ of the main peak. 


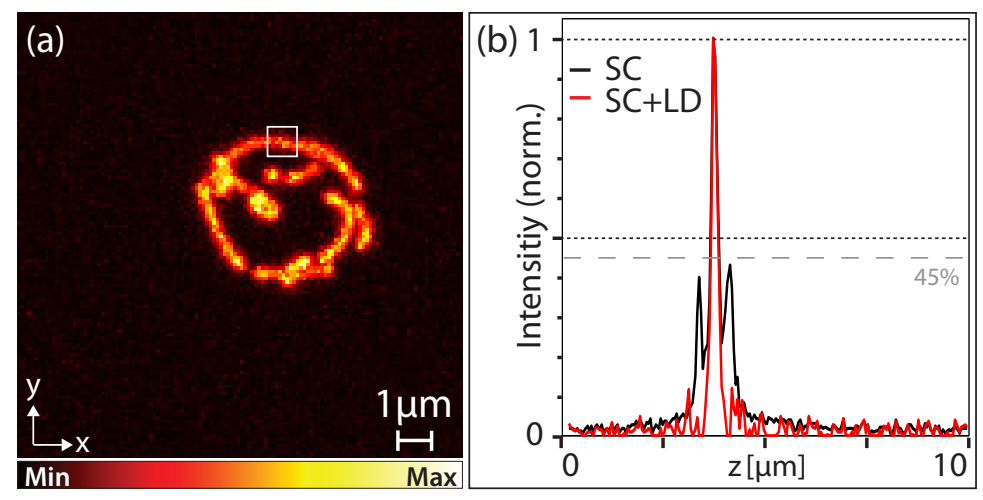

Figure 3.8: MMM 4Pi type A measurement with post-processing-confocalization of GFP-tagged mitochondria in fixed budding yeast. (a) shows the maximum projection of a stack of $200 \mathrm{xy}$ slices which were post-processed with the 'superconfocal'-algorithm. The total acquisition time was $25 \mathrm{~s}$. (b) depicts the axial profile (black line) of the region marked in (a). The side lobes have a relative height of $40 \%$ and can therefore be removed by linear $5 p$ deconvolution (LD) (red line).

The linear deconvolution of the summed and interpolated data with a $100 \mathrm{~nm}$ bead reveals an axial resolution of at least $125 \mathrm{~nm}$.

The applicability of the MMM 4Pi type A mode in conjunction with post-processingconfocalization to studies of biological samples was next shown by the measurements of fixed and live budding yeast cells.

The maximum projection of a stack of 200 xy slices of a fixed budding yeast cell is shown in figure 3.8 (a). The total acquisition time which was mainly comprised of the camera exposure time and the axial scan time accumulated to $24 \mathrm{~s}$ for one 3D stack. Panel (a) shows just a part of the whole lateral field of view. Each slice was reconstructed with the 'superconfocal'-algorithm from a set of 40 images. In order to minimize the data volume, the calculation was carried out simultaneously to the recording which increased the total acquisition time of the complete stack time only by $4 \%$ to $25 \mathrm{~s}$. Figure 3.8 (b) shows the axial profiles of the region marked in (a). The side lobes have a relative height of $40 \%$. They can therefore be unambigously removed by a linear $5 \mathrm{p}$ deconvolution.

Figure 3.9 shows a time series of ten 3D stacks of a live budding yeast cell. The stacks were recorded in a row so that the time lag between the starting times of consecutive stacks was $25 \mathrm{~s}$. One cannot fully exclude mitochondrial movement within the recording time of one stack. However, as one xy image extends over the whole mitochondrial compartment, putative movements would have only affected the $\mathrm{z}$ direction. After acquisition, the data was linearly $3 \mathrm{p}$ deconvolved, smoothed and volume-rendered (Amira, Visage Imaging, Inc., San Diego, CA, USA). The fast data acquisition permits observation of a mitochondria-matrix separation event (' ', , figure 3.9 (c)-(e)) and a fusion event ('**', figure $3.9(\mathrm{~h})-(\mathrm{j}))$. 


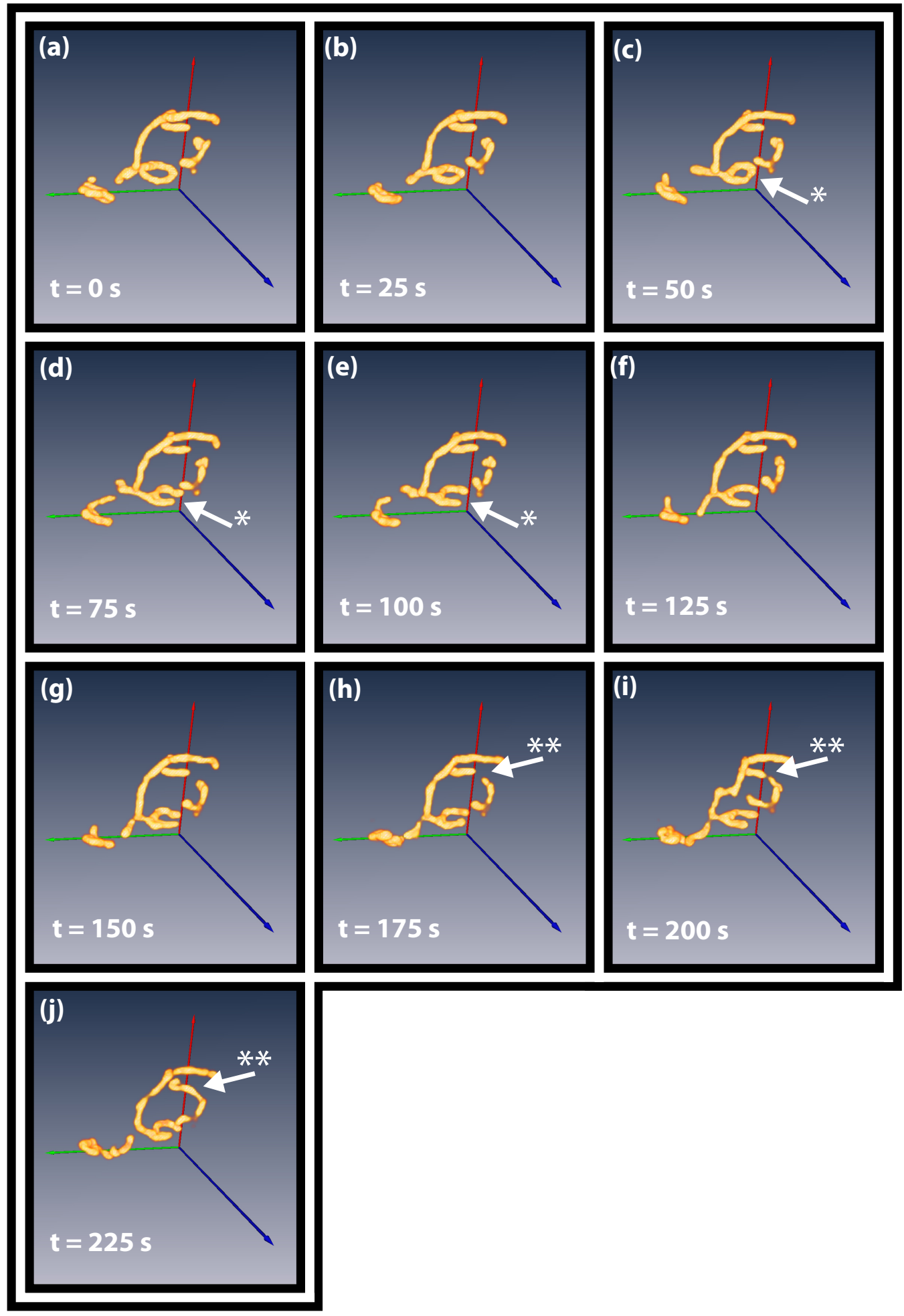

Figure 3.9: 3D volume-rendered illustration of the temporal dynamics of GFP-tagged mitochondria in a living budding yeast cell. The images (a)-(j) were recorded in MMM 4Pi type A mode with post-processing-confocalization and were processed by the 'superconfocal'-algorithm. The recording time for each stack was $25 \mathrm{~s}$ including axial scanning and post-processing. The arrows highlight a mitochondria fission ('*') and a fusion ('**') event. The axial direction is indicated by the red arrow. Figure adapted from [59]. 



\section{Single marker switching microscopy}

\subsection{Introduction to high resolution switching-based microscopy}

Since the late 19th century, it has been common knowledge that light cannot be focused to an arbitrarily small spot which implicates that the resolution in far-field light microscopy is restricted due to Abbe's diffraction limit (cf. chapter 2) [1]. While chapter 3 has illustrated how to push this seemingly fundamental limit by increasing the solid aperture angle with two opposing lenses, this section will present means to overcome the barrier by spatio-temporal separation of identical markers.

The basic idea to break the resolution limit is to use the marker not as a mere contrast agent, but to employ its specific properties. Resolution enhancement can be achieved if the marker features two different states, which can be distinguished from each other, and if the transition between these states can be controlled at least unidirectional. The distinct states can be states which are absorbing and non-absorbing, fluorescent and non-fluorescent, in general signaling and non-signaling. Switching between these two states implies an (optical) transition which renders one state quasi non-occupied. This can be achieved if the driven transition outperforms all other competing transitions which depends on the lifetimes of the transitions involved. The concept of diffraction-unlimited far-field optical microscopy has been implemented by employing several strategies which have their own strengths and limitations.

In STED (stimulated emission depletion) microscopy, the first excited electronic state is depleted by stimulated emission [60] [11]. To this end, a spatial intensity distribution of a wavelength at the upper range of the emission spectrum effectively inhibits fluorescence emission, except at a region where the distribution features a zero intensity node. Because of the short lifetime of the fluorescent state, STED calls for comparatively large beam intensities of $\mathrm{GW} / \mathrm{cm}^{2}$.

In GSD (ground state depletion) microscopy, the ground state is emptied by a saturated transition to a metastable dark state [16] [17]. Fluorescence is confined to the zero node of an intensity distribution which can achieved with relatively modest intensities of several $\mathrm{kW} / \mathrm{cm}^{2}$.

Surpassing the diffraction barrier with illumination intensities which are in the range of $10 \mathrm{~W} / \mathrm{cm}^{2}$ can be achieved by reversibly photoswitching marker proteins between fluorescent and non-fluorescent conformational states [18]. 
These approaches, which have been generalized under the acronym RESOLFT (reversible saturable optically linear (fluorescence) transitions) [15] [10], confine the signaling state to a region which is well-defined by the zero region of a spatial intensity distribution. The diffraction-limited image is assembled sequentially in time by translating the pre-known location of the zero over the sample and therefore by switching ensembles of markers at defined position ('targeted signal switching'). During the scanning process, the fluorophores undergo several switching-cycles.

In the next sections a complementary high-resolution concept which involves stochastic switching of single markers will be discussed in detail.

\subsection{Breaking the diffraction barrier by switching single markers}

Analogous to the 'targeted' concepts (e.g. STED) which were briefly discussed in section 4.1, resolution enhancement in SMS (single marker switching) microscopy is based on switching between signaling and non-signaling states (cf. figure 4.1 (a)) [10]. These states will be referred to as on-state and off-state, respectively. In principle, like in the 'targeted' concepts, the signal is not restricted to fluorescence. However, fluorescence will be assumed in the following because all implementations so far have used it as a signal.

Despite switching being the common basic principle, there are several differences between the targeted read out and SMS microscopy. In the latter, markers at random positions are recorded individually rather than in an ensemble. Multiple switching cycles of single markers are therefore not needed. The basic concept will be explained in detail with the aid of figure 4.1. Let the markers be photoswitchable, featuring a dark state and a bright state (figure 4.1 (a)). If, like in conventional fluorescence microscopy, the fluorescence of all these markers is imaged simultaneously, sub-diffraction sized features of the object cannot be resolved anymore (figure 4.1 (b)). Let us now exploit the markers' photoswitching properties and assume that initially all markers are either already in their off-state or can be driven to it (figure 4.1 (c)). The markers are then exposed to switching light of an intensity which is chosen such that only few random markers are transferred to the on-state at a time. This subset must be so small that the probability of two switchedon markers being closer than the diffraction-limit is negligible. Upon excitation, markers of this small fraction can emit many fluorescent photons in a row which will be referred to as burst or event in the following. Neighboring markers which reside in their off-state remain entirely dark. The read out of adjacent identical markers is therefore separated in time. Since a single marker is already the smallest possible emitter, no further optical confinement is necessary to reduce the fluorescent region. When recording the fluorescence photons on a pixilated detector such as a camera, every fluorescent marker is blurred to a diffraction-limited spot by the imaging system. Unlike in the targeted concepts in which the position of the fluorescent emitters is defined by the zero-intensity region of the 


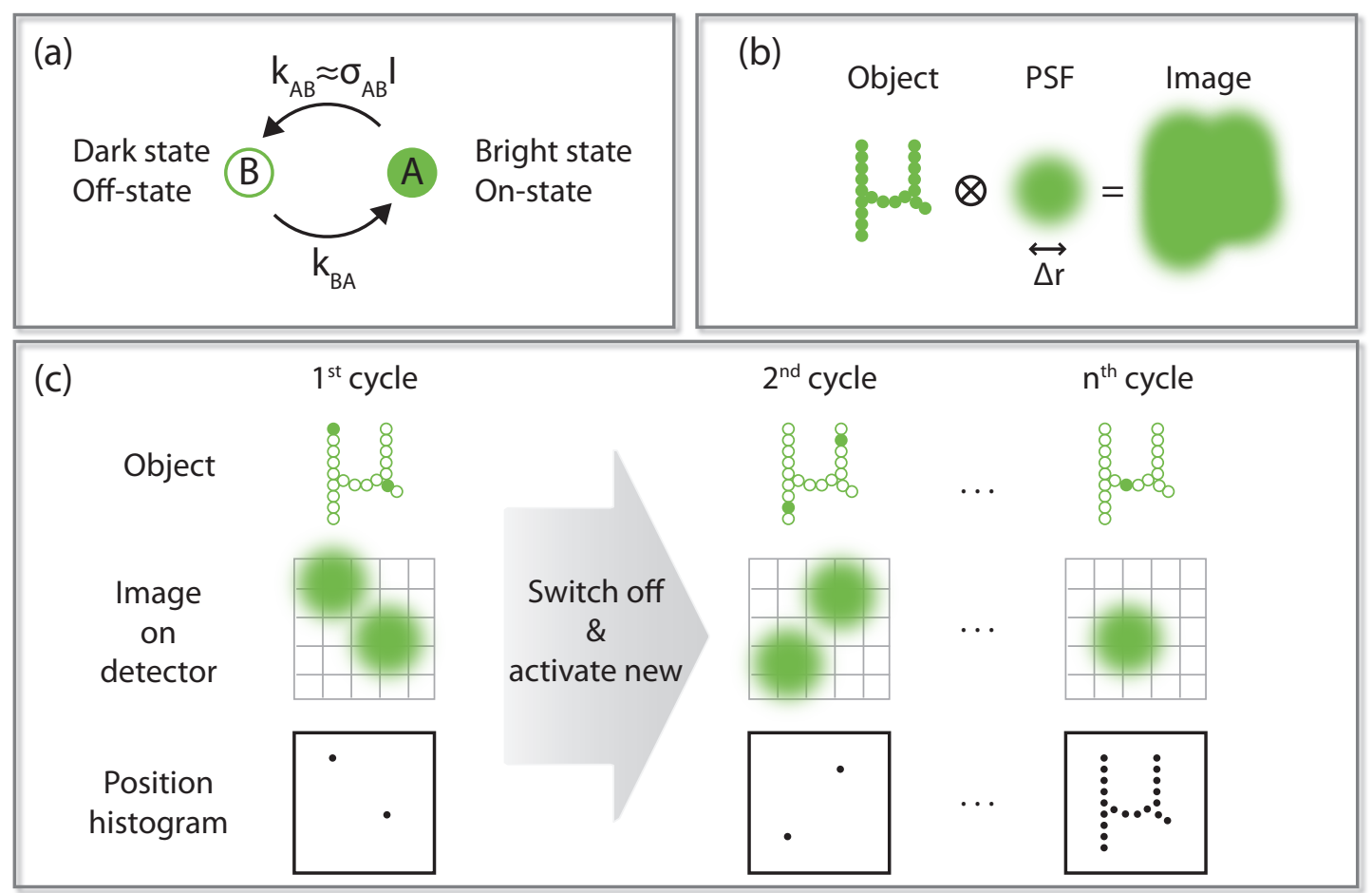

Figure 4.1: Basic concept of SMS microscopy. (a) Simplified scheme of the marker with two distinguishable states. Here, the bright state A (filled green circle) is fluorescent. The transition to the dark state B (hollow green circle) is induced by an intensity I. k denotes the rate constant, $\sigma_{\mathrm{AB}}$ denotes the cross-section of the transition from A to B. (b) The object is composed of individual markers which are in their bright state. Imaging the marker distribution is described as a convolution of each individual marker with the PSF. If the markers' positions are closer than the resolution $\Delta \mathrm{r}$, their images overlap and the object's sub-diffraction sized features cannot be resolved anymore. (c) Spatio-temporal separation of the markers. Top left: Only a small subset of markers is in its bright state and emits fluorescence when being excited. Middle left: Their images do not overlap on the pixilated detector if the switched-on markers are further apart than $\Delta r$. Bottom left: The center positions are determined and plotted in a position histogram. After imaging, the markers are switched off and a new subset is activated ( $2^{\text {nd }}$ cycle). This is repeated until enough marker positions have been registered to reconstruct the object ( ${ }^{\text {th }}$ cycle). 
light distribution, the position of the emitting marker is unknown. However, the a priori knowledge that the photons in one diffraction-limited spot stem from one single marker allows calculating the position of the emitter with much higher accuracy than the FWHM of the imaging system's PSF [61] [62] [63] [64] [65]. After the markers are switched off, bleached or transferred to another dark state, meaning that the marker underwent one switching cycle, a new subset of markers at random positions can be activated, excited and their positions can be subsequently localized. This process is repeated until enough marker positions are registered to get a sufficient representation of the marker distribution in the sample. The final image is assembled by simply marking the positions of the localized emitters.

This single molecule recording method was termed photoactivated localization microscopy (PALM) [19], fluorescence photoactivation localization microscopy (FPALM) [20] or stochastic optical reconstruction microscopy (STORM) [21]. In the course of this thesis, all these concepts will be referred to by the hypernym SMS microscopy for simplicity.

As pointed out above, multiple switching cycles are not needed which eases the requirements on the markers in terms of switching fatigue. However, there are particular requirements on the marker's properties which are summarized and explained here:

- The marker must exhibit at least two distinguishable states. The signal of these states is not limited to fluorescence.

- At least one transition between these states must be controllable. As mentioned above, controlling the transitions is not restricted to light. Other possibilities like thermally or chemically induced transitions can be thought of as well. In the following, light-induced transitions will be assumed. In case of irreversibly photoswitchable fluorophores, usually the on-transition is light-induced. Switching off is done by photobleaching of the fluorophores. This implies that these markers can undergo only one switching cycle and can therefore be read out only once. Reversibly photoswitchable emitters can undergo several cycles which means that they are available for multiple read out.

- The signal contrast between the distinguishable states must be high. Since the detection of single molecules implies detection of relatively low light levels, any remaining signal of the high number of surrounding switched-off markers adds up and might severely disturb the detection and the subsequent localization.

- The marker must emit a preferably high number of photons in a preferably short period of time. The more photons are detected for one marker at a time, the more exact its position can be determined (cf. section 4.3.4 for further detail). The shorter the time period in which these photons are detected, the less time is needed for the total image acquisition. 
- The ratio between markers in their on-state and markers in their off-state must be low. If it is too high for a given density of fluorophores, switched-on markers will be closer than the diffraction-limit. A too high ratio therefore restricts the labeling density of the structures of interest. The ratio depends on the rate constants for the transitions between the available states of the marker [20].

\subsubsection{Photon statistics}

While the number of photons of a coherent light source follows a Poisson distribution, the number of photons which is emitted by single molecules follows a geometrical distribution.

Let a marker have a the bright state $\mathrm{A}$ and the dark state $\mathrm{B}$ and let the on-transition $\mathrm{B} \rightarrow \mathrm{A}$ be light-induced with a rate constant $k_{\text {on }}\left(I_{\mathrm{on}}\right)$, where $I_{\text {on }}$ denotes the light intensity. The reverse transition is spontaneous with a rate constant $k_{\text {off }}$. When being in the onstate, the marker can be excited to the first electronic state. From there, several pathways for transitions are available: the marker can either emit a fluorescence photon with a rate constant $k_{\mathrm{fl}}=1 / \tau_{\mathrm{fl}}\left(\tau_{\mathrm{fl}}\right.$ denotes the fluorescence lifetime), it can be photobleached $\left(k_{\text {bleach }}\right)$ or it can be transferred to a long-living dark state $\left(k_{T}\right)$, typically a triplet state. For the fluorophores which are used in this thesis, it can be assumed that the spontaneous transition with $k_{\text {off }}$ is negligible. This leaves the other two pathways with $k_{\text {dark }}=k_{\text {bleach }}+$ $k_{T}$ which compete with the fluorescence transition. This means that for each excitationemission cycle, there is a certain probability $p=p_{\text {dark }} /\left(p_{\text {dark }}+p_{\mathrm{fl}}\right)=k_{\text {dark }} /\left(k_{\text {dark }}+k_{\mathrm{fl}}\right)$ that the marker is driven to a state in which it cannot fluoresce. Consequently, the probability $P$ for emitting $N_{e}$ photons is

$$
P\left(N_{e}\right)=p \cdot(1-p)^{N_{e}} .
$$

The number of photons which are extracted from one molecule burst thus follows a geometrical distribution. An exemplary distribution is depicted in figure 4.2 (b). It is a histogram of the numbers of photons of approximately 18,000 registered and localized events which is fitted by an exponential function (red). Note the truncation of the geometrical distribution at the lower range of the $\mathrm{x}$ axis. The reason for this is that the analysis routine applies a photon threshold on the registration of the events, which is typically well above the noise level, to ensure that noise is not falsely identified as an event.

\subsubsection{Positioning accuracy}

The image of the photon burst which stems from one point-like object is blurred to a diffraction-limited spot on the detector. Its size and shape is defined by the microscope's detection PSF $h_{\text {det }}(x, y)$ which can be approximated by a 2D Gaussian $h_{\text {det }}(r)=A$. $\exp \left(-\frac{\left(r-r_{0}\right)^{2}}{2 \sigma_{\text {det }}^{2}}\right)$, where $r=x^{2}+y^{2}, \sigma_{\text {det }}$ denotes the standard deviation of $h_{\text {det }}$ and $A$ denotes the amplitude. Figure 4.2 (a) presents a typical frame of a SMS measurement 
(a)

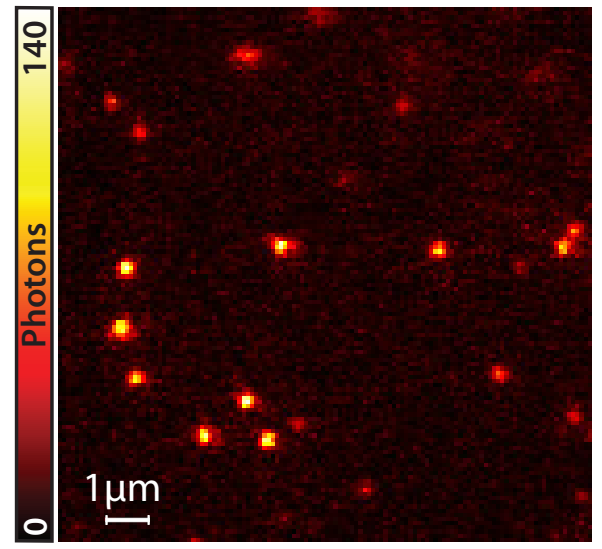

(b)

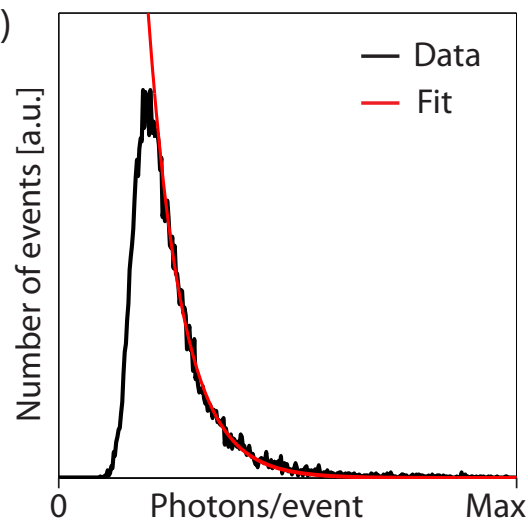

Figure 4.2: (a) Typical frame of a SMS measurement. Fluorescence of single synthetic fluorophores is recorded on a CCD chip. Each diffraction-limited spot is created by photons emitted by one molecule within the camera exposure time of $10 \mathrm{~ms}$. Even by eye, it becomes obvious that the center position of each photon distribution can be determined better than its width. Note the different total brightness values of the spots. (b) Exemplary histogram of the detected photons per event (data was taken from the measurement shown in 4.7). It can be very well fitted by an exponential decay function (red). Note the truncation at low photon numbers which is due to a thresholding process in the analysis routine (cf. section 4.3.4).

and, even by eye, it becomes clear that the center positions of the spots can be estimated more exactly than the width of the spot.

The localization accuracy of the image's center position depends on the number of collected photons. Each photon which is collected is a sample for the marker's position. The position error is the standard deviation $\sigma_{d e t}$ of the detection PSF $h_{\text {det }}$ of the imaging system. According to common statistics, the standard deviation of the mean is given by

$$
\Delta r=\frac{\sigma_{d e t}}{\sqrt{N}}
$$

where $N$ is the number of detected photons.

In practice, this means that if a marker at coordinate $r$ emits several bursts with $N$ photons each, the center position estimates for each burst will be normally distributed around $r$ with a standard deviation of $\sigma_{d e t} / \sqrt{N}$. It is usually more convenient to measure the accuracy in terms of the FWHM instead of the standard deviation because the former is a more intuitive measure: $\Delta r_{\mathrm{FWHM}}=2 \sqrt{2 \ln 2} \cdot \Delta r \approx 2.35 \cdot \Delta r$. Equation (4.1) implies that $\Delta r$ can decrease arbitrarily for $N \rightarrow \infty$.

Except for shot noise, equation (4.1) neglects all other sources of noise like background noise, pixelation noise, dark counts or readout noise. Dark noise, which is thermally induced in the detector in the absence of light, is typically negligible if the CCD chip is operated at low temperatures. Background noise should be reduced to a minimum, e.g. by applying appropriate optical filters. Readout noise is an inherent output amplifier noise and can be neglected when using an EMCCD device. This amplifies the signal 
before reading out so that readout noise is no longer a limit on sensitivity and is usually effectively less than one electron. Due to this electron multiplying gain, an additional excess noise factor of approximately $\sqrt{2}$ is introduced [66]. The theoretical localization accuracy is therefore

$$
\widetilde{\Delta r}=\frac{\sigma_{d e t}}{\sqrt{N / 2}}
$$

when using an EMCCD camera chip.

Pixelation noise stems from the uncertainty where the photon hits the pixel. According to Thompson et al. [67], the optimum pixel size is $\sigma_{\text {det }}$. A coarser sampling results in a loss of information, while a finer one deteriorates the SNR. This is because the signal is spread onto more pixels, while the noise per pixel remains constant.

\subsubsection{Image representation}

After having discussed the photon distribution and the photon dependent localization accuracy, it becomes obvious that for each emitter two parameters can be derived: its position $r$ and the localization accuracy $\Delta r(N)$ (cf. equation (4.1)). Because of the geometric distribution of the photon numbers, there is not only one localization accuracy present in a high resolution SMS image. Note that since the accuracy describes the spatial probability distribution of the localized centers, one can also use the term PSF. Therefore, instead of having just one PSF, the PSF with the worst accuracy experiences admixtures of narrower PSFs which are less probable. Please refer to [68] for a detailed analysis of the effective PSF in SMS microscopy.

It is intuitive to specify a minimum localization accuracy for the lowest photon number or a mean localization accuracy for the average photon number. These values can be tuned by introducing a thresholding procedure such that only events with photon numbers exceeding $N_{T}=\sigma_{\text {det }}^{2} / \Delta r_{0}^{2}$ are taken into account. Hereby, the minimum occurring precision is set to $\Delta r_{0}$ by rejecting all events with a worse accuracy. Obviously, a gain in precision in the image is obtained at the expense of the number of events which are accepted for the representation of the sample. This trade-off between accuracy and density of the image is a comfortable mean to match, for example, the accuracy to the half of the minimum structure size to achieve an optimum sampling [37]. Alternatively, the positioning accuracy can be downgraded to achieve a smoother representation of the object. However, one must keep in mind that increasing $N_{T}$ results in a fast loss of events due to the geometric distribution of the photon numbers per event. When increasing $N_{T}$ from $N_{T_{0}}$ to $N_{T_{1}}$, the minimum localization accuracy is improved by a factor of $\sqrt{N_{T_{1}} / N_{T_{0}}}$. Concomitantly, the number of events in the final image decreases by a factor of $\exp \left(q^{-1} \cdot\left(N_{T_{1}}-N_{T_{0}}\right)\right)$ where $q$ denotes the expectation value of the exponential fit to the geometric distribution. For example, when increasing the photon threshold from $N_{T_{0}}=250$ to $N_{T_{1}}=500$ photons (factor 2), the minimal accuracy improves only slightly from 7.7 to $5.4 \mathrm{~nm}$ (factor 1.41), while the number of events decreases 2.7 -fold. This examples assumes $\sigma_{d e t}=121 \mathrm{~nm}$ and $q=250$ photons. Two potential problems arise if $N_{T}$ is set too high. First, the image might appear spotty which can be compensated by increasing the acquisition time provided that 
the labeling density of the sample is high enough. Second, if the average brightness of the markers is not space-invariant, for example, due to different local environments, regions with higher average photon number will be emphasized.

So far three different types of representation have been reported which differ in their displayed information content.

The first approach presents the accumulated marker positions. This means that the pixel value in the final image corresponds to the number of markers whose localized positions fall into the respective pixel. According to [68], this view is referred to as 'histogram' view. A slight modification is the 'smoothed histogram' view in which each event is presented by a Gaussian which is centered at the localized center position. Its FWHM is fixed at a constant value and its integral over the area is unity. The Gaussian filtered version leads to a smoother appearance of the data. Care has to be taken that the smoothing does not involve any unwanted loss of positioning information. The '(smoothed) histogram' view weights all events equally. However, it disregards the accuracy information.

The second type of representation which can be seen in [19] was named 'Gaussian' view [68] and presents each registered event as a Gaussian spot with a FWHM which is equal to the theoretical positioning accuracy according to equation (4.1). The Gaussian is normalized such that the integral over the area is unity. This approach displays both localization and precision information in one representation. The effective PSF of the 'Gaussian' view is broader than the 'histogram' PSF which is illustrated by Middendorff et al. [68].

The third approach bins the events according to their positions. The pixel value is the sum of all photon numbers of the corresponding events. An example of this 'brightness' view can be seen in [69]. This view attempts to include the brightness information additionally to the position information. However, as the number of localized events per pixel cannot be deduced from the image, the brightness information does not help in assessing the precision information. The 'brightness view' increases the apparent dynamic range compared to the 'histogram' view.

\subsection{SMS microscopy in two dimensions}

Despite the fact that SMS microscopy is a powerful technique [10], the first demonstrations of this novel nanoscopy form entailed a number of limitations. Since it relies on the detection of single markers [70], it is very sensitive to diffuse background signal so that background suppression techniques such as the total internal reflection (TIRF) imaging were usually employed [19] [21][71][72]. This limited the method to thin samples, namely to sectioned slices thinner than $100 \mathrm{~nm}$ [19]. Moreover, extremely long measurement times of 2 - 12 hours were reported for acquiring a meaningful image [19]. In the following, a fast asynchronous acquisition protocol along with a novel fast reversibly photoswitchable fluorescent protein (RSFP) [73] will be introduced. This allows one to cut down the measurement time 100-fold, to image from the interior of intact cells and to simplify the experimental setup. 


\subsubsection{Imaging protocol with independently running acquisition}

So far, long camera exposure times [19][20] and/or measurement protocols [19][21], which synchronized the switching on - reading out - switching off/bleaching cycle with the camera, had been implemented. The involved drawbacks stated in the preceding section have been overcome by improving the imaging protocol which will be described in the following.

\section{The novel fast switching reversibly switchable fluorescent protein}

The novel fast switching variant (V157G) [73] of the reversibly switchable fluorescent protein Dronpa [74] is employed. The key event of the photochromic switching process is a cis-trans isomerization of the chromophore. By a point mutation, which reduces the steric hindrance for the conformational change, the energy barrier for the transition to the non-fluorescent trans-state is lowered. Hereby, the switching to this state of this new variant rsFastLime was accelerated by a factor of 50 compared to Dronpa [73]. It emits green fluorescence around $500 \mathrm{~nm}$ and is excited by $488 \mathrm{~nm}$ light which also induces the switching off process. Here, the term switching off shall denote all processes which lead to a temporary or permanent loss of the ability to fluoresce. The reverse transition from the dark to the bright state is driven by near UV to blue light.

\section{The optimum match}

Due to a switching on cross-section of the $488 \mathrm{~nm}$ light, termed switch-on crosstalk, the blue light not only excites, i.e. reads out the protein, and switches off, but also induces the on-transition. Since both the switching on and the switching off occur from excited states, the associated rates are proportional to the excitation intensity, $I$, for moderate intensities. This means that not only the density of molecules in their on-state, but also the number of photons emitted from the fluorophore, is independent of the intensity, while the excitation-emission cycling time is proportional to $1 / I$. Consequently, by increasing $I$, the average on-time of a marker $t_{\text {on }}$ can be reduced dramatically while retaining the same event density and the same number of fluorescence photons, i.e. the same localization accuracy. This degree of freedom allows the matching of the average burst length $t_{\text {on }}$ to the highest frame rate $f$ at which the detection camera can maintain a nearly $100 \%$ duty cycle. This measure optimizes the acquisition protocol not only in terms of speed, but also in terms of the background signal. While frame rates higher than $1 / t_{\text {on }}$ spread the signal over several frames and therefore increase the readout noise and the dark counts, lower frame rates result in an unnecessary accumulation of background signal.

\section{The experimental effort}

As mentioned above, $488 \mathrm{~nm}$ light is sufficient for inducing both the switching on and the reading out which simplifies the experimental effort. The accompanying loss of one degree of freedom for tuning the number of switched-on markers by independent activation can be generally compensated by adjusting the labeling density within certain limits. 
This is margined by the maximum possible labeling density of a structure imposed by the labeling protocol and by the minimum density which sufficiently marks the structures of interest. Moreover, if the density is still too high meaning that an unambiguous assignment of detected photons to single emitters is not guaranteed, pre-bleaching is an option as well as applying an excitation wavelength with less switching crosstalk. Conversely, a density which is too low can be improved by increasing the excitation density beyond optimum $\left(t_{\text {on }}<1 / f\right)$.

\section{The asynchronous protocol}

SMS microscopy relies on the stochastic switching on of markers throughout the field of view. The new strategy introduces additionally a stochastic read out in time, as opposed to former implementations which used a fixed temporal sequence of switch on, read out and bleaching [19]. The sample is continuously illuminated. Therefore, switching on occurs at arbitrary times during image acquisition which makes the synchronization of illumination and image acquisition redundant. Since spontaneous off-on-off cycles of individual switchable fluorophores are recorded non-triggered and without synchronizing the detector, the protocol was first published under the acronym PALMIRA (photo-activated localization microscopy with independently running acquisition) [75][76]. In the following, it will be referred to by the generic term SMS microscopy for simplicity.

\subsubsection{Single molecule spectroscopy}

For evidencing the general applicability of the fast asynchronous imaging protocol (along with the RSFP rsFastLime) for SMS microscopy, a sparse sample with rsFastLime was continuously illuminated by $488 \mathrm{~nm}$ light. The freely running camera operated at a frame rate of $500 \mathrm{~Hz}$ with a duty cycle of $97 \%$. Section 4.3 .3 provides a detailed description of the experimental implementation. The sample was prepared as follows. rsFastLime was expressed and purified as described previously [73]. Microscope cover slips were rinsed with deionized water for 5 min and cleaned in a low pressure plasma system (Femto-RF, Diener Electronic, Nagold, Germany). Purified rsFastLime (1.27 nM) was dissolved in a phosphate buffered saline (PBS) based solution ( $\mathrm{pH} 7.4)$ with $0.1 \%(\mathrm{w} / \mathrm{v})$ polyvinyl alcohol (PVA) (88 mol\% hydrolyzed, Polysciences Europe, Eppelheim, Germany) and $0.32 \%$ (w/v) L-ascorbic acid (A.C.S. reagent, Sigma-Aldrich, St. Louis, MO, USA). A $40 \mu \mathrm{l}$ aliquot of the solution was pipetted onto the coverslip which was then spin-coated for $20 \mathrm{~s}$ at $3000 \mathrm{rpm}$ (KW-4A, Chemat Technology, Northridge, CA, USA). The overall image acquisition time was $40 \mathrm{~s}$ and the light intensity was $5.0 \mathrm{~kW} / \mathrm{cm}^{2}$.

The maximum projection of all 20,000 frames (cf. figure 4.3 (d)) discloses the presence of some sparsely distributed isolated objects. They are most likely single molecules as the concentration of the markers was only in the $\mathrm{nM}$ range and because the localized positions did not show any indication that they represent more than one molecule. Figure 4.3 (a)-(c) presents three typical frames from the whole series of frames in which the excellent SNR becomes evident. The root mean square of the combined background and readout noise 

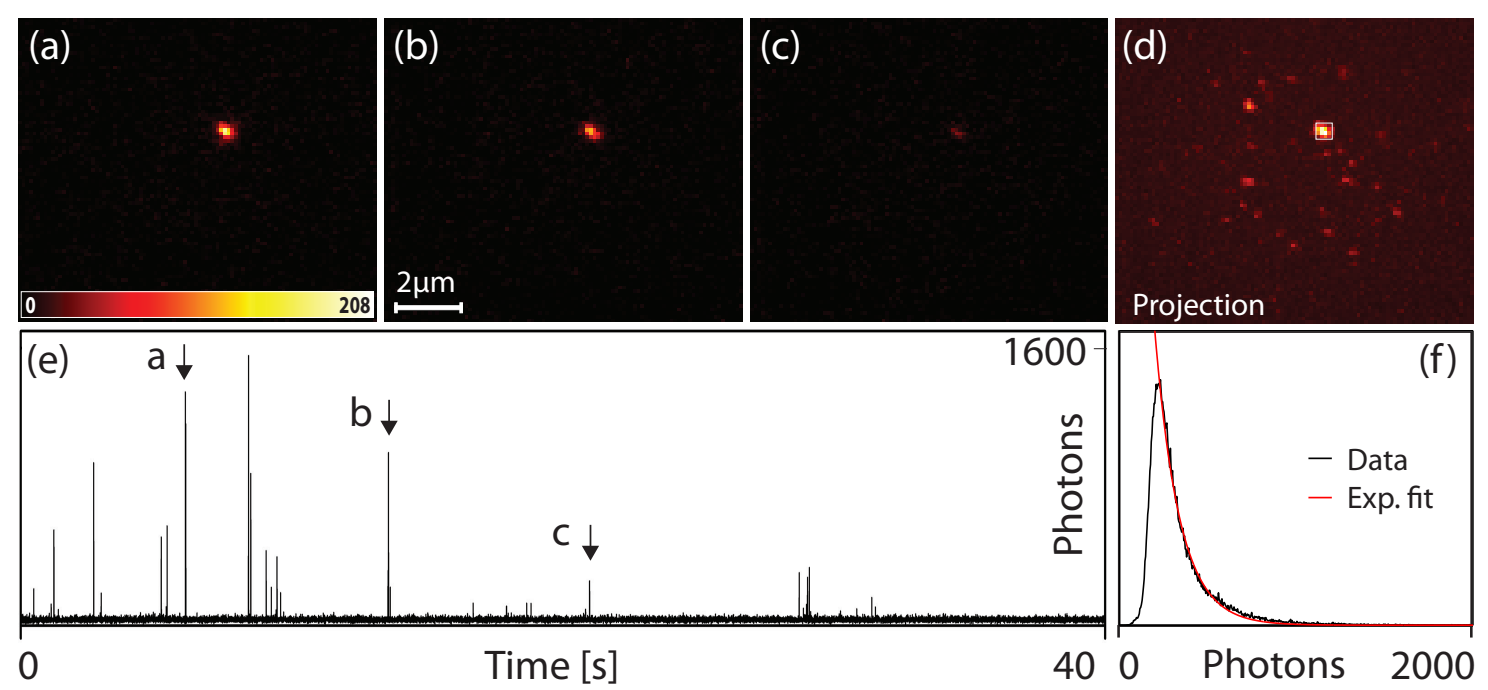

Figure 4.3: Imaging single rsFastLime molecules with continuous illumination by $488 \mathrm{~nm}$ light. (a-c) Three typical recordings of a sparse rsFastLime sample. (d) Maximum projection from the whole series of 20,000 frames. The object labeled by the white square is most likely a single molecule. (e) The time trace integrated over the $4 \times 4$ pixels marked in (d) shows that the object is switched on and off over 25 times. The arrows mark the bursts corresponding to the frames shown in (a)-(c). (f) The distribution of photons per single molecule event can be well approximated by an exponential decay of first order for large photon counts. The expectation value is approximately 140 photons.

averaged to less than one photon per pixel. When tracking over time the region, which is marked by the white rectangle in 4.3 (d), it becomes evident that the illumination by mere $488 \mathrm{~nm}$ light induced all transitions as expected (panel (e)). Fluorescence from a marker was detected for several short time intervals which means that the marker was repeatedly switched between the fluorescent on-state and a non-fluorescent state. Since switching as well as bleaching is a stochastic process, it is not clear whether the object was bleached or switched off at the end of the series of frames.

In addition to high resolution position information, further analysis gives insight into more single molecule properties. Here, this shall be exemplified for the photon number distribution, which is not available in ensemble fluorescence microscopy methods, and the on-time of rsFastLime in PVA. Additionally, other single molecule characteristics like for example polarization states [77] and spectral properties [78] have been investigated with SMS microscopy.

Evaluation of about 600 time traces gives an estimate of the fraction of on-time in the equilibrium state under $488 \mathrm{~nm}$ illumination. By dividing the number of frames in which a marker was visible by the number of frames, until the last fluorescence emission of the marker was detected, $0.2 \%$ was obtained as the upper bound for the probability of a rsFastLime protein to be in its on-state. Figure 4.3 (f) presents the distribution of photon numbers per single molecule event, which can be well approximated for large 


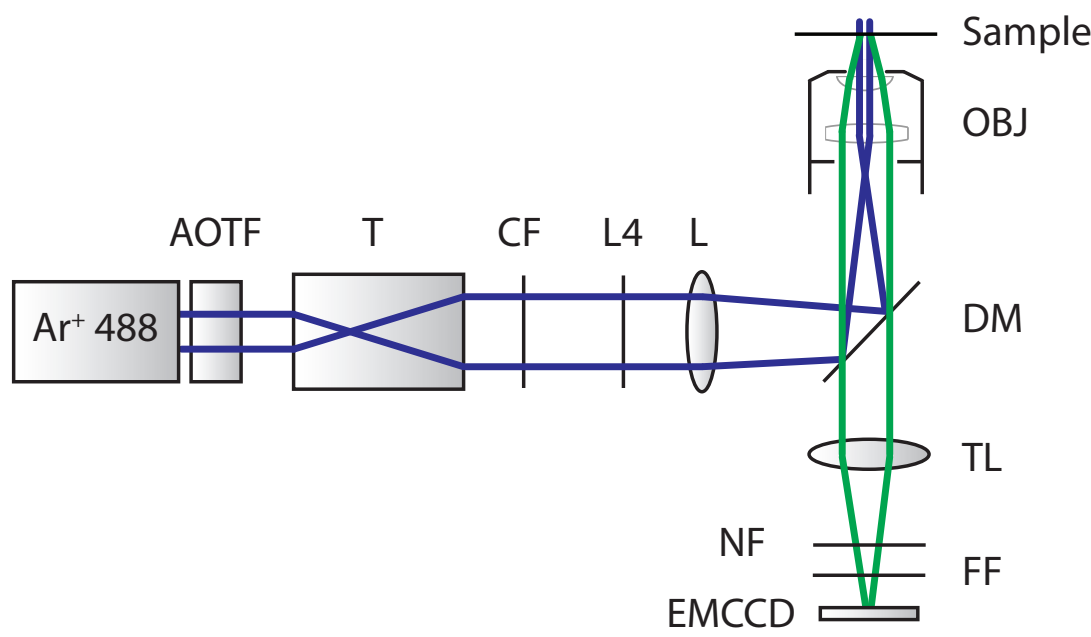

Figure 4.4: Epifluorescence setup for SMS microscopy with independently running acquisition. An argon ion laser source $\left(\mathrm{Ar}^{+} 488\right)$ provides the light (blue line) for the wide-field illumination of the sample. The fluorescence (green line) is imaged onto a pixilated detector (EMCCD). AOTF: acousto-optical tunable filter, T: telescope, CF: clean-up filter, L4: $\lambda / 4$ waveplate, L: lens, OBJ: objective lens, DM: dichroic mirror, TL: tube lens, NF: notch filter, FF: fluorescence filter.

photon numbers by an exponential decay with an expectation value of approximately 140 photons.

\subsubsection{Experimental implementation}

The experimental arrangement for the SMS measurements with independently running acquisition was based on a wide-field imaging setup and is illustrated in figure 4.4. The excitation laser light was provided by an argon ion laser (Innova 300, Coherent, Santa Clara, CA, USA). An acousto-optical tunable filter (AOTF, AA Optoelectronic, Orsay cedex, France) selected the $488 \mathrm{~nm}$ laser line and controlled its intensity. The laser light was spectrally cleaned up (Z488-10, Chroma Technology, Rockingham, VT, USA), expanded by a telescope and converted from linear to circular polarization by a quarter wave plate. Subsequently, the beam was directed into the side port of a commercial wide-field microscope (DMIRE 2, Leica Microsystems, Wetzlar, Germany). Uniform epiillumination of a field of view of approximately $10 \mu \mathrm{m}$ in diameter was achieved by under-illuminating the back-aperture of the objective lens (HCX APO 100x/1.30 Oil U-V-I 0.17/D, Leica Microsystems, Germany). The fluorescence was collected by the same lens and decoupled from the excitation beam path by a dichroic mirror (Z495DCXR, AHF Analysentechnik, Tübingen, Germany). It was further cleaned up from residual laser light and background light outside the fluorophore's emission spectrum by a notch filter (DNPF488-25, LOTOriel, Darmstadt, Germany) and a band-pass filter (HC525/50, Semrock, Rochester, NY, USA) and subsequently imaged onto an EMCCD camera (Ixon plus DU-860, Andor Technology, Belfast, Northern Ireland). If necessary, this setup can easily be modified to 


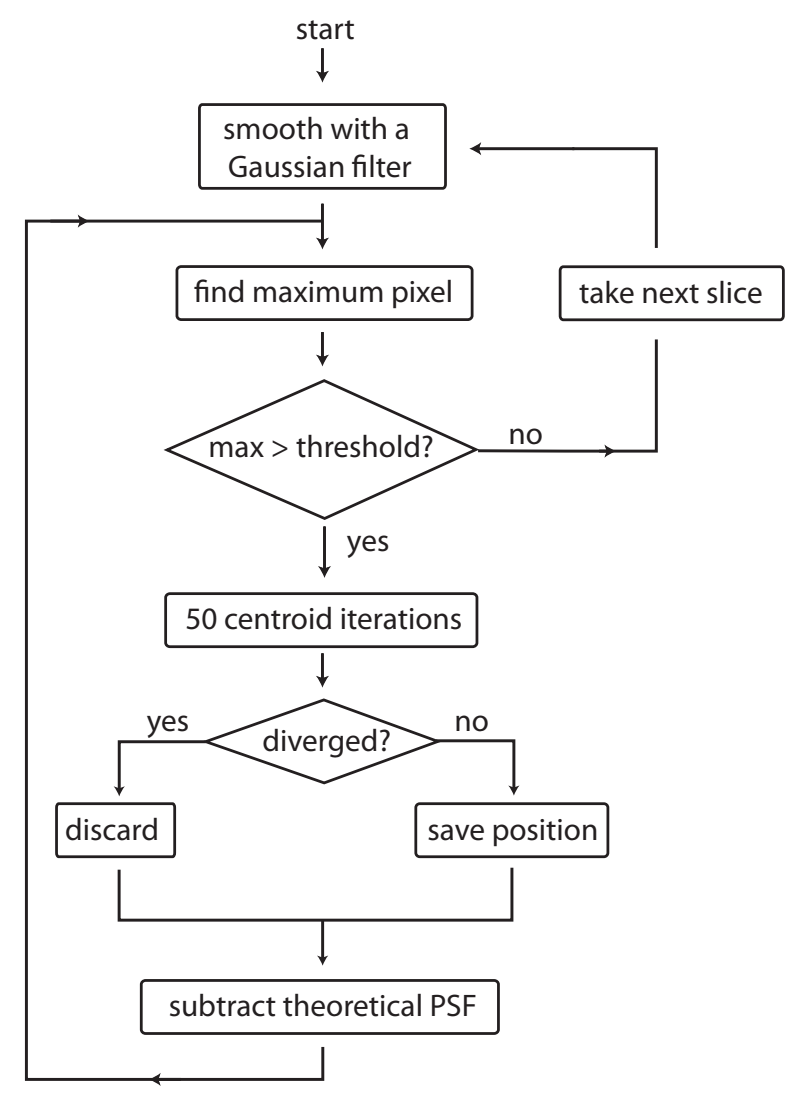

Figure 4.5: The combined CLEAN/mask-fitting algorithm to identify and localize single emitters in individual camera frames. Each image is initially smoothed by a Gaussian filter that is much narrower than the PSF. Subsequently, the pixel with the highest photon count is identified and used as a starting point for the mask-fitting iteration described in the text. If this iteration converges, the retrieved $2 \mathrm{D}$ position of the marker is tabulated together with the number of photons. A theoretical PSF is then subtracted from the image. The algorithm then reiterates, identifying the pixel with the highest photon count. When this photon count is below a certain threshold value, which is chosen well above background level to avoid artifacts, the next frame is taken. The flow diagram is adapted from [76].

accommodate additional laser lines which will be presented in section 4.4.2.

\subsubsection{Positioning in the lateral direction}

Once the images have been recorded in a wide-field setup as described in section 4.3.3, the markers are identified and localized by a combination of a segmentation algorithm and a mask-fitting algorithm of the Airy spot. The applied segmentation routine is close to Högbom's classical CLEAN algorithm [79]. The Gaussian mask-fitting algorithm which was derived from a simplified least-squares analysis is intermediate in complexity between a full non-linear least-squares fit and a centroid-based algorithm. It is equivalent to the algorithm described by Thompson et al. [67].

Figure 4.5 depicts the flow diagram of the interplay of the two algorithms. First, a Gaussian filter is applied to the frame. The size of the Gaussian is chosen well below the width of an Airy spot. The resulting noise reduction is sufficient to provide notably better segmentation without compromising the localization precision. Next, the pixel with the highest photon count is identified. If no pixel count exceeds the threshold, the whole frame is discarded and the next frame is taken. The center $\vec{r}_{0}$ of the brightest pixel serves as the starting point for the localization routine. In every iteration, the center of mass $\vec{r}_{n}$ is calculated from the data multiplied by a Gaussian which is centered at $\vec{r}_{n-1}$. The 
width of the Gaussian is matched to the width of the detection PSF. The algorithm usually converges after a few iterations. If after 50 iterations the distance $\vec{r}_{n}-\vec{r}_{0}$ is greater than the width of the Airy spot, the event is discarded. Otherwise, the $2 \mathrm{D}$ position along with the photon number of the event are registered for further representation. The CLEAN algorithm then entails the subtraction of a theoretical signal which leads to a superior treatment of slightly overlapping events compared to simple connection-based segmentation algorithms. The amplitude of the theoretical Gaussian is chosen such that after subtraction the pixel count at the marker's position becomes zero above the median background. The segmentation and localization is repeated for each frame of the measurement.

During the typical image acquisition time of 2-2.5 min, a sample drift of some tens of nanometers was observed. For that reason, fluorescent microspheres were added to the samples. By tracking these bright particles (typically more than 2,000 photons per frame), the errors in the determined positions of individual fluorophores can be corrected during the post-processing analysis.

\subsubsection{High resolution 2D images of technical and biological specimens}

In order to demonstrate the practicability of the novel RSFP rsFastLime [73] along with the optimized imaging protocol described in section 4.3 for high resolution imaging, technical and biological samples were investigated.

At first, the imaging of nanofabricated structures, in particular grooves which were milled into fused silica cover slips, is presented. Figure 4.6 (c) shows the inverted height profile of the nanostructures as recorded with an atomic force microscope (AFM). Even though the tip was not sharp enough to resolve the profile of the grooves completely, it is clearly visible that there are six parallel furrows separated between 235 and $315 \mathrm{~nm}$. After AFM imaging, the substrate was stained by spin-coating a PBS-based solution with $1.15 \mathrm{nM}$ rsFastLime, $0.05 \%$ (v/v) fluospheres (FluoSpheres carboxylate-modified microspheres, $0.2 \mu \mathrm{m}$, Nile red fluorescent, Invitrogen, Carlsbad, CA, USA), $0.8 \%$ (w/v) PVA and $0.29 \%(\mathrm{w} / \mathrm{v})$ L-ascorbic acid. Since PVA accumulated in the grooves during the spin-coating process, a labeling of this region was expected due to the higher density of rsFastLime proteins. This assumption was confirmed by the conventional image shown in figure 4.6 (b). This image was obtained by summing up all recorded frames and setting the lowest pixel value to zero, because in case of negligible readout noise, the epifluorescent analogon to the superresolved SMS is given by the sum of the individual frames. The pixelated impression of the conventional image is due to the fact that the pixel size was chosen such that it is optimal for the localization algorithm. As Thompson et al. have pointed out, the pixel size should be approximately the standard deviation of the PSF in order to optimize the localization uncertainty [67]. A finer sampling leads to a smoother appearance of the data, however one does not gain any more information ('over-sampling'). The diffraction-limited resolution in figure 4.6 (b) is not high enough to distinguish the single 

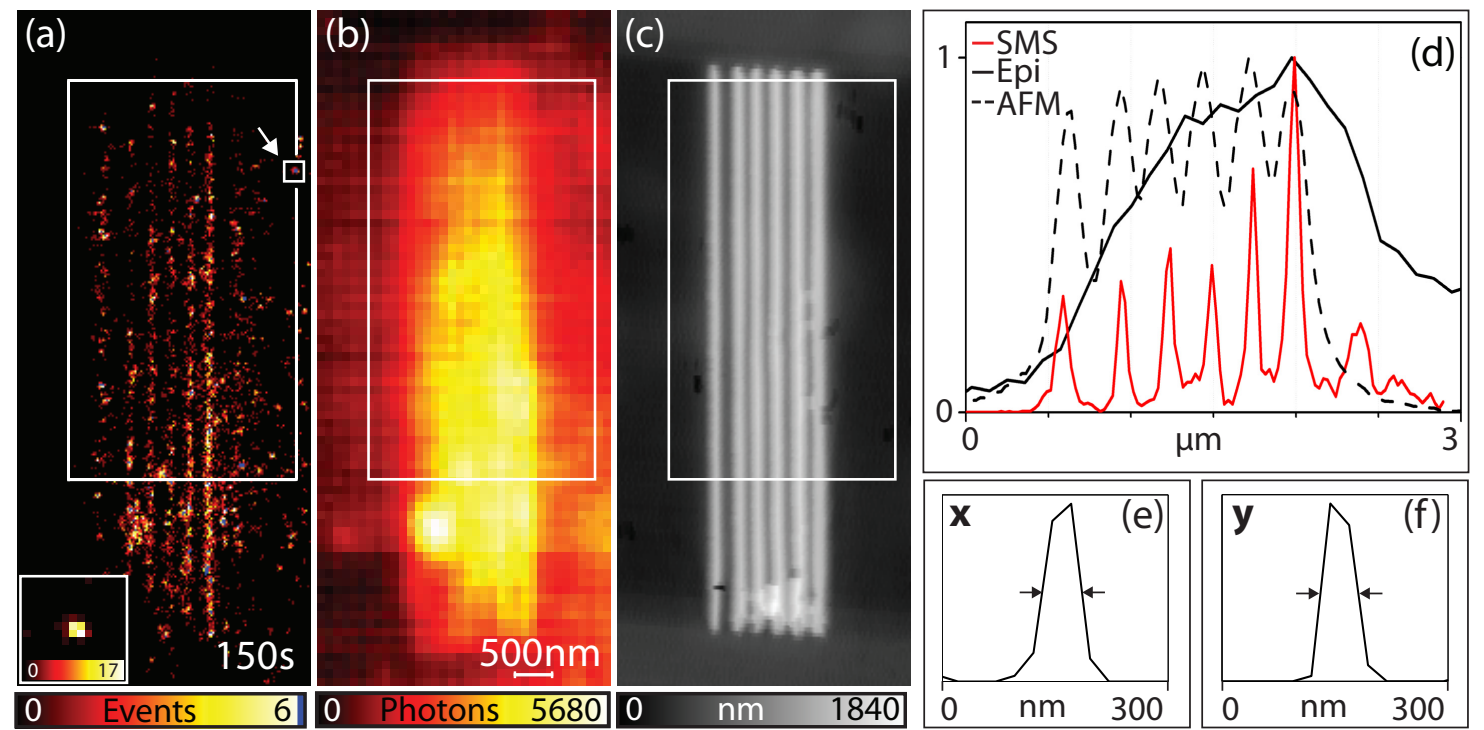

Figure 4.6: Imaging of grooves milled into a cover slip. (a) SMS, (b) reconstructed epifluorescence and (c) inverted AFM image. Profiles integrated over the area indicated in (a-c) are shown in (d). The inset in (a) magnifies the rectangle marked by the arrow and shows the SMS image of a small agglomeration of several rsFastLime proteins. Its extent $(e, f)$ serves as a conservative estimate for the positioning accuracy.

furrows. In contrast, they are clearly resolved in the SMS image presented in figure 4.6 (a). It was generated by analyzing 75,000 frames which is equivalent to an acquisition time of $150 \mathrm{~s} .9426$ events are shown in the 'histogram' view with a pixel size of $25 \mathrm{~nm}$. The illumination intensity was $2.4 \mathrm{~kW} / \mathrm{cm}^{2}$. The atomic force micrograph and the high resolution image differ slightly because the height profile of the structures is not necessarily equivalent to the protein distribution. The additional peak as seen in the intensity profile in panel (d) was likely caused by inhomogeneities in the spin-coating process. In order to assess the localization accuracy in the SMS image, the object marked by the white rectangle, most likely showing an agglomeration of several proteins, was analyzed. The FWHM of the averaged $\mathrm{x}$ and y profiles (panels $(\mathrm{e}, \mathrm{f})$ ) is approximately $50 \mathrm{~nm}$ which presents the lower bound for the localization accuracy.

This experimental platform is not restricted to the imaging of technical samples. $200 \mathrm{~nm}$ thin cryosections of cytoplasmic membrane labeled $E$. coli were obtained and spin-coated with a $40 \mu \mathrm{l}$ aliquot of a PBS-based solution ( $\mathrm{pH} 7.4$ ) with $0.045 \%(\mathrm{v} / \mathrm{v})$ fluospheres and $0.09 \%(\mathrm{w} / \mathrm{v})$ PVA. Please refer to the appendix for information about the fusion protein and the preparation of thin cryosections (obtained by Dirk Wenzel, Department of Neurobiology, Max Planck Institute for Biophysical Chemistry, Göttingen, Germany). In figure 4.7 (a), all localized protein positions whose photon number exceeded 88 photons are binned into $20 \mathrm{~nm}$ pixel. The illumination light intensity was $5 \mathrm{~kW} / \mathrm{cm}^{2}$. The acquisition time of approximately $140 \mathrm{~s}$ yielded enough events (ca. 22,000) to form clear images 


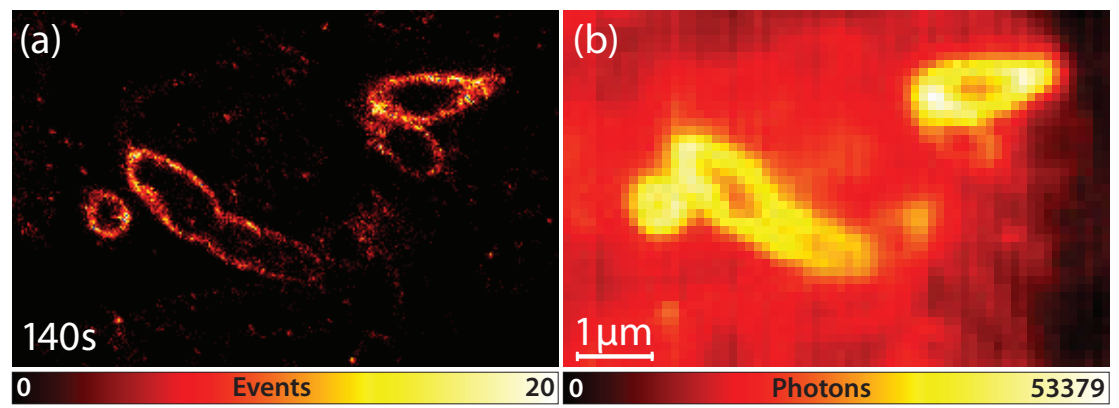

Figure 4.7: Imaging E. coli. (a) SMS and (b) reconstructed conventional image of a $200 \mathrm{~nm}$ thick cryosection of cytoplasmic membrane labeled E. coli. The SMS image was recorded in $140 \mathrm{~s}$.

with sufficient dynamic range. Several bacteria can be identified whose membranes are much more clearly resolved than in the reconstructed conventional image in figure 4.7 (b).

Due to one of the key features of the fast imaging protocol (cf. section 4.3.1), which is matching the camera exposure time to the average duration of the photon burst, the background was minimized. Thereby, dedicated background suppression techniques (like TIRF microscopy) became obsolete. Consequently, there was no fundamental reason anymore to be limited to thin cryosections or surfaces whose images were presented in the preceding section. While the signal stems from markers which are located inside a volume in which the detection PSF does not significantly change and while the number of fluorescent molecules per unit area is sufficiently small, it is also possible to localize markers in whole cells or even thicker samples.

For this purpose, labeled $\alpha$-tubulin inside whole PtK2 cells was imaged. Please refer to the appendix for details on the staining procedure. For sample preparation, a PBS-based solution with $0.045 \%$ (v/v) fluospheres, $0.09 \%$ (w/v) PVA and $0.29 \%$ (w/v) L-ascorbic acid was prepared. The $\mathrm{pH}$ was adjusted to 7.4-7.5. A $15 \mu \mathrm{l}$ aliquot of this solution was pipetted onto the sample which was then spin-coated for $40 \mathrm{~s}$ at $3000 \mathrm{rpm}$.

Figure 4.8 compares the reconstructed conventional (panel (a)) and the SMS (panel (b)) image of the same region inside the whole cell. The SMS image was generated by analyzing 60,000 frames. The overall image acquisition time was $120 \mathrm{~s}$ and the light intensity was increased stepwise from $3.5 \mathrm{~kW} / \mathrm{cm}^{2}$ to $5.0 \mathrm{~kW} / \mathrm{cm}^{2}$. While individual microtubules are not resolved in the conventional image, they can be clearly distinguished in the SMS recording. The resolution is even high enough to pinpoint the somewhat inhomogeneous labeling along the filaments. The distribution of detected photons per event for the data presented in figure 4.8 (b) is shown in panel (e). Due to the threshold of six photons in the CLEAN algorithm, detection of events with a small number of photon counts was significantly less probable. For large photon counts per event $(\geq 120)$, the histogram follows a geometrical distribution with an expectation value of approximately 84 photons. In oder to estimate the positioning accuracy, the object indicated in figure 4.8 (b) was scrutinized which probably represents an agglomeration of several rsFastLime proteins. The 


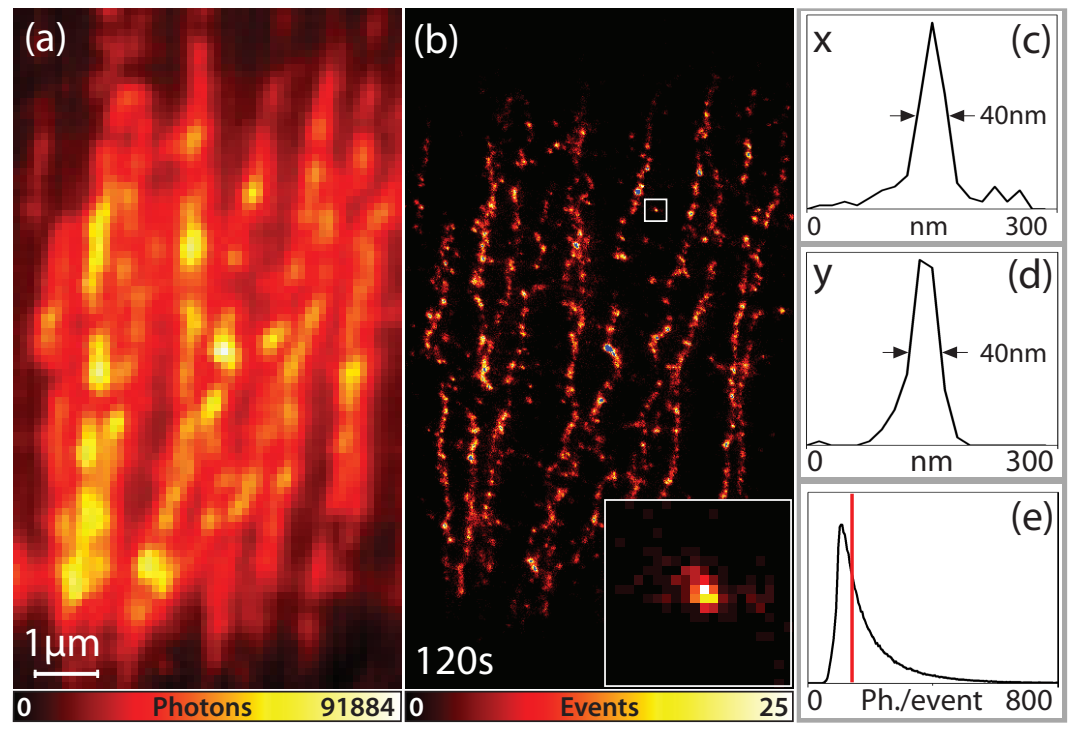

Figure 4.8: Imaging stained $\alpha$-tubulin inside intact PtK2 cells. (a) Reconstructed conventional image and (b) SMS image which was recorded in $120 \mathrm{~s}$. The averaged line profiles in $\mathrm{x}$ (c) and $y$ direction (d) of a single rsFastLime molecule or agglomeration of molecules (inset in panel (b)) prove a focal plane resolution of $40 \mathrm{~nm}$. (e) The distribution of detected photons per single molecule event for large photon counts $(\geq 120)$ is well approximated by an exponential decay function with an expectation value of approximately 84. Only events with 140 photons or more (red vertical line) were chosen for the data representation.

averaged $x$ and y profiles in panel (c) and (d) reveal that the resolution can be tuned to approximately $40 \mathrm{~nm}$ by only choosing events, whose photon number exceeds 140 photons, for the data representation. This fits well to the theoretical lower limit for the precision of $34 \mathrm{~nm}$ given by equation (4.2) for a PSF with $285 \mathrm{~nm}$ (FWHM). The slight difference might be due to a lateral extent of the agglomeration of markers. Note that the color values in the images represent the number of single molecule events per pixel. Consequently, the resolution directly results from the statistics of recording many events. Higher thresholds would improve the resolution further, but reduce the dynamic range.

\section{Summary}

To summarize the preceding sections, nanoscale far-field fluorescence imaging was demonstrated based on reversible photoswitching and detecting the photon bursts of single markers. Acquisition times of 2-2.5 min were sufficient to get meaningful images. These enhancements are due to an asynchronous laissez-faire protocol of data recording that just matches the camera exposure time to the duration of the photon bursts. The demands on the long-term stability of the experimental setup are eased by the 100 -fold reduction in acquisition time compared to previously published experiments. Dedicated background suppression techniques such as TIRF imaging are no longer generally necessary for single 
molecule, localization-based applications. Consequently, the use of a standard epifluorescence setup allowed the imaging of features inside whole cells.

Because of the reversibility of the switching process and because a photon burst may be spread over several frames, it is possible that a marker contributed to the final image more than once. Nevertheless, the overcounting probability is equal for every marker, ensuring that the final image is an unbiased representation of the fluorophore distribution in the specimen. It is not required to know or control the average number of total counts. The switching reversibility may even be advantageous as molecules which have emitted not enough photons for being registered have another chance to contribute to the image. Also time-lapse recordings will likely benefit from the reversibility because the staining density is less probably changed by the read out of the markers. Nevertheless, SMS with independently running acquisition will perform just as well with irreversibly switching fluorophores.

\subsection{Extension to the third dimension}

As illustrated in section 4.3, high localization accuracy down to the molecular scale can easily be achieved with a simple experimental scheme and (non-)commercial marker molecules. However, the world is not composed of only 2D structures. When imaging 3D biological or technical structures, it is recommendable to provide localization or at least optical sectioning in the remaining axial direction. Without this axial information, signal from the depths of the sample will deteriorate the contrast in the imaging plane.

Optical sectioning combined with switching and subsequent 2D localization enables SMS microscopy with high lateral localization accuracy in 3D thick samples. To this end, $2 \mathrm{P}$ activation with pulsed or continuous wave lasers confines the region in which molecules can be switched to their on-state to the vicinity of the focal plane [80] [81] [82]. Hereby, diffuse background from out-of-focus events and unnecessary switching cycles are avoided. This method provides major improvement. However, the single events are not localized in the axial direction, but all detected events are only assigned to the focal plane.

For positioning single emitters in the axial dimension, an estimation procedure for their $\mathrm{z}$ position is needed. This is somewhat more demanding than for the lateral positions because the shape rather than the position of the detected patterns changes. For example, when using wide-field detection, the degree of blurring of the detected spot gives a rough estimate for the axial distance from the focal plane. However, estimates close to the focal plane are very inaccurate because the detection PSF varies only slightly with the emitter's axial position. Even worse, the images of emitters at equal distance below and above the focal plane are indistinguishable because of the symmetry of the detection PSF. Therefore, axial localization relies on detection schemes which break the symmetry of the PSF.

A straightforward way to distinguish between positions above and below the focal plane is to introduce an astigmatism which can be easily implemented by placing a cylindrical lens into the detection beam path [83]. This leads to an elliptical intensity distribution 
with its principal axis being aligned to one lateral direction or the other depending on the emitter's axial position. A different approach is to sample the PSF in the axial direction with two offset channels. This scheme will be referred to as 'defocused' detection in the course of this thesis.

Very recent approaches which shall not be discussed in detail here are 4Pi-related methods [84] [85] or the PSF-engineering shown by Pavani et al., who apply double-helix PSFs [86].

\subsubsection{Axial positioning - defocus method}

In order to break the symmetry of the detection PSF, the signal may be split into two (or more) non-aberrated detection channels whose focal planes are at a certain distance from each other. Both the defocused and the astigmatic detection scheme have their own peculiarities. Whereas the former one has an isotropic lateral localization accuracy, the latter features a higher complexity in radial localization because $\mathrm{x}$ and $\mathrm{y}$ are no longer equivalent. The defocused scheme requires the distribution of the signal into at least two channels, while in the astigmatism-based approach one channel is already sufficient which may be advantageous if readout noise is not negligible. On the other hand, polarization effects have been found to feature elliptically deformed intensity distributions [87], which may lead to false axial localization when relying on astigmatic aberrations. Moreover, when imaging in thicker samples, spherical aberrations induced by a refractive index mismatch have to be treated adequately [88].

Analysis of the localization accuracy using the Fisher information matrix elucidates that both methods show a trade-off between axial and lateral accuracy [84]. With increasing strength of the astigmatism, the axial localization accuracy improves near the focal plane while the lateral accuracy becomes anisotropic and deteriorated. In the defocused scheme, the lateral one remains isotropic. However the broadening of the PSF increases the FWHM and therefore worsens the lateral accuracy. Free parameters for tuning this trade-off are the number of channels and the distance of the focal planes. The investigation of the Cramer-Rao bounds (lower estimate for the localization accuracy) reveals that the astigmatic scheme does not outperform the defocused scheme in $\mathrm{x}, \mathrm{y}$ and $\mathrm{z}$ at the same time [84]. This result is supported by experimental findings which further indicate that the defocused result outperforms the aberrated one by a factor of two in terms of the axial range in which localization can be performed [89].

The defocused scheme has recently been applied to high resolution localization-based microscopy imaging $4 \mu \mathrm{m}$ diameter beads (BP FPALM, [69]). By means of astigmatic aberrations, high resolution imaging of intracellular structures in whole fixed cells was demonstrated (3D STORM, [83] [88]).

In the course of this thesis, a defocused scheme was implemented by modifying the detection path of the experimental setup shown in subsection 4.3.3. Switching was performed by either $1 \mathrm{P}$ or $2 \mathrm{P}$ activation and the first $3 \mathrm{D}$ SMS images of intracellular structures in living cells were recorded. 


\subsubsection{Imaging setup and image registration}

For 3D SMS imaging, the wide-field 2D setup shown in figure 4.4 was expanded and modified in several ways. In order to render the system more flexible with respect to the use of different fluorophores, each with its distinct absorption and emission spectrum, additional lasers for excitation and (1P, 2P) switching were introduced. Additionally, the detection path was adapted to the defocused detection scheme by introducing two detection channels. They were imaged onto the same camera chip, which avoided synchronization problems between separate cameras. The implementation of more than two channels would have improved the homogeneity of the axial localization at the cost of the lateral one. However, the gain in accuracy did not justify the additional complexity of the experiment.

For the excitation of a wide range of fluorophores, an optically pumped semiconductor laser (Sapphire 488LP-50mW, Coherent Inc., Santa Clara, CA, USA) running at $488 \mathrm{~nm}$, a diode pumped solid-state laser (LC-532-200, Shanghai Laser Century Technology Co., Shanghai, China) at $532 \mathrm{~nm}$, as well as a $560 \mathrm{~nm}$ fiber laser (VFL-P-560-1000, MPB Communications Inc., Montreal, Quebec, Canada) were available. The laser light which was delivered to the sample was intensity-controlled by an acousto-optical tunable filter (AOTFnc-VIS-TN, AA Optoelectronic, Orsay cedex, France), expanded by a telescope and spectrally cleaned up if necessary (488/10 and Z532/10x, Chroma Technology, Rockingham, VT, USA). For the switching the fluorophores, either a $375 \mathrm{~nm}(1 \mathrm{P}$ switching) (Cube 375-16C, Coherent Inc., Santa Clara, CA, USA), a $405 \mathrm{~nm}$ (1P switching) (DL100T, Toptica) or a Ti:Sa laser (2P switching) (Chameleon XR, Coherent Inc., Santa Clara, CA, USA) running at $740 \mathrm{~nm}$ were used. The blue/green excitation laser beams and the UV laser light were combined at a dichroic mirror (Z415 RDC, AHF Analysentechnik, Tübingen, Germany) and they were added to the $2 \mathrm{P}$ laser light (628 dc lpxr, AHF Analysentechnik, Tübingen, Germany). For switching between the three excitation lasers and the two UV activation lasers, respectively, magnetically coupled mirror holders were used. All laser beams were converted from linear to circular polarization by quarter wave plates and subsequently coupled into a regular commercial wide-field microscope body (DMIRE 2, Leica Microsystems, Wetzlar, Germany). Uniform epiillumination of the field of view with the UV and the blue/green lasers was assured by under-illuminating the back-aperture of the objective lens (HCX APO 1003/1.30 Oil U-V-I 0.17/D, Leica Microsystems, Germany). The $2 \mathrm{P}$ laser beam however was split into several beamlets by a disk of microlenses (each of focal length: $12 \mathrm{~mm}$ ) in a Nipkow type arrangement. Their foci acted as point sources for the $2 \mathrm{P}$ activation and were imaged into the sample. Scanning of the foci in the lateral direction was accomplished by rotating the microlens disk which resulted in a uniform movement of the point sources over the field of view. Moving the sample in the axial direction was done by a single axis nano-positioning system (Nano Z200, Mad City Labs Inc., Madison, WI, USA) which was integrated into a home-built stable scanning block and sample holder. Lateral movement of the sample was done with the help of two piezo-electric motors (Piezo LEGS, PiezoMotor, Uppsala, Sweden; Motion 


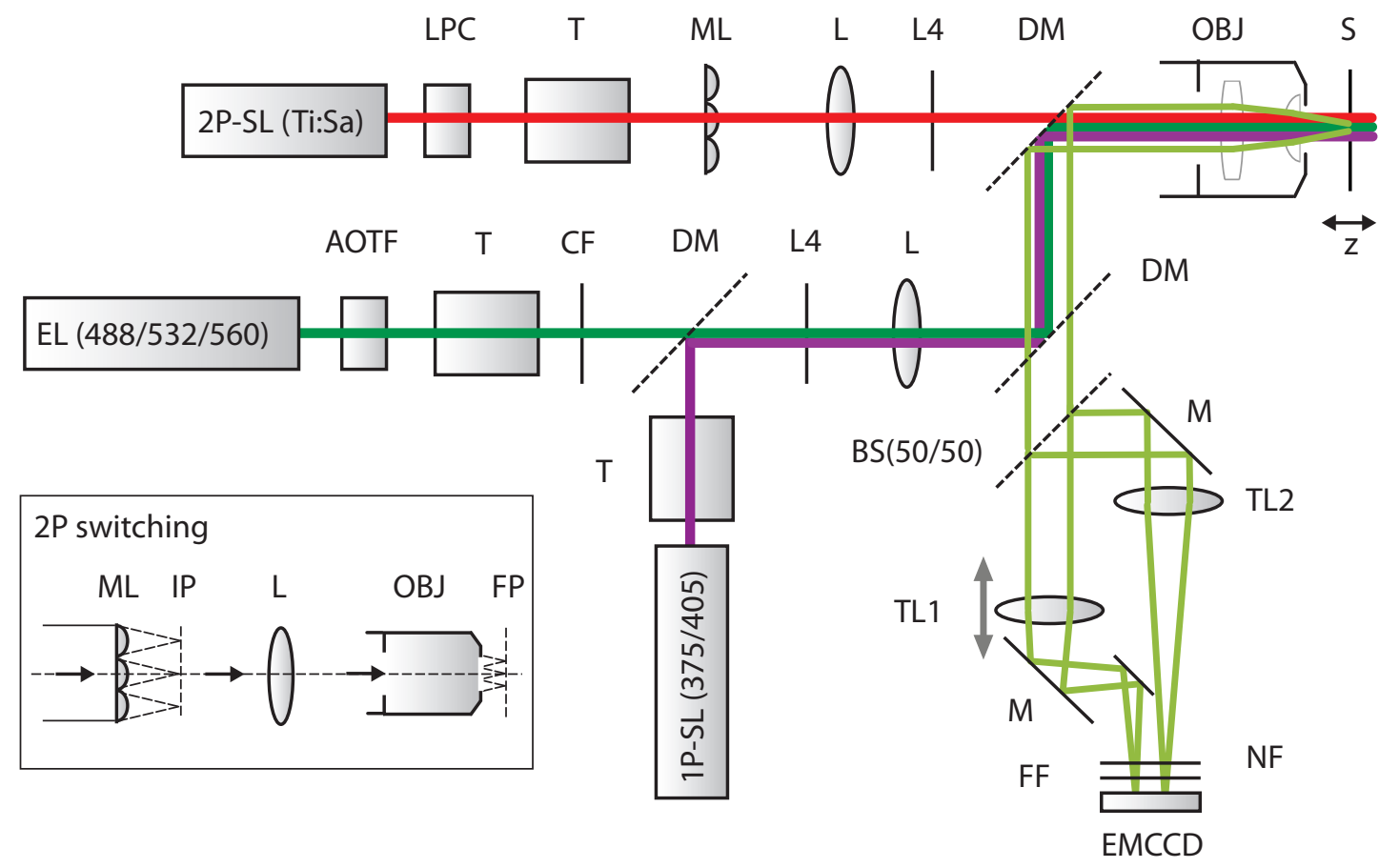

Figure 4.9: Implementation of the 3D SMS experiment. Several laser lines are available for excitation (green line) (EL (488/532/560)), 1P switching (purple line) (1P-SL (375/405)) and 2P switching (red line) (2P-SL (Ti:Sa)). While the first two are implemented using a wide-field illumination, the pulsed $2 \mathrm{P}$ light is split into several beamlets whose foci are imaged into the sample (see inset). Fluorescence (light green line) is collected by the same objective lens, split by a beam splitter (BS(50/50)) into two parts of equal intensity. Each of the two is imaged onto the EMCCD chip (EMCCD) by a tube lens (TL1 and TL2, respectively). One tube lens (TL1) can be moved along the axial direction in order to offset the focal planes with respect to each other. LPC: laser power control, T: telescope, L: lens, L4: $\lambda / 4$ waveplate, S: sample, DM: dichroic mirror, AOTF: acousto-optical tunable filter, M: mirror, CF: clean-up filter, NF: notch filter, FF: fluorescence band-pass filter. Please refer to the text for further details. Inset: 2P switching. The 2P light is split into several beamlets by a spinning microlens disk (ML). Their foci are imaged into the focal plane (FP) of the objective lens (OBJ) and induce 2P switching. IP: image plane. 
Commander, NANOS-Instruments GmbH, Hamburg, Germany). The fluorescence emitted by the sample was collected by the same objective lens, separated from the laser light by a dichroic filter (Z495DCXR; Z532/NIR RPC; Z580DCXR, all AHF Analysentechnik, Tübingen, Germany) and subsequently split into two beams by a 50/50-beam splitter. Both beams were then directed onto different areas of the same EMCCD camera chip (Ixon plus DU-860, Andor Technology, Belfast, Northern Ireland; later replaced by Ixon plus $D U$-897) by two independent imaging lenses. Stray laser light and background outside the dye's emission spectrum was removed by a notch filter (DNPF488-25, LOT-Oriel, Darmstadt, Germany; NF01-532U-25; NF01-568U-25 (tilted), Semrock, Rochester, NY, USA) and a suitable band-pass filter (HC525-50; FF01-582/75-25;FF01-630/92-25, all Semrock, Rochester, NY, USA).

By shifting the two imaging lenses in opposite directions from their original position, the respective focal planes move closer to and further away from the objective. The imaged area is bordered by a rectangular aperture which is placed into a back-projected object plane in the shared beam path. In other words, the same object area of the sample is imaged twice onto the camera chip with two different axial planes being in focus. Two different cameras were used for the 3D SMS measurements.

The first EMCCD camera in use (Ixon plus, DU860) had a quadratic chip with 128x128 pixels which was divided horizontally into two channels. Since one pixel corresponded to $96 \times 96 \mathrm{~nm}^{2}$ in the object plane, the field of view was rectangular $\left(12.3 \times 6.15 \mu \mathrm{m}^{2}\right)$. The (1024x1024 pixels) chip of the second camera (Ixon plus, DU897) was not read out completely, instead a $2 \times 2$ binned area of $222 \times 111$ pixels was used which resulted in a quadratic field of view of $14.7 \times 14.7 \mu^{2}$ (one pixel corresponded to $132 \times 132 \mathrm{~nm}^{2}$ ).

Figure 4.10 (a) exemplifies a frame of a typical 3D SMS measurement. The pixels of the lower half are attributed to channel 1, the pixels of the upper half to channel 2. The dotted white line marks the separation between the two channels. The initial localization is carried out for all pixels by an algorithm which is similar to the one described in section 4.3.4. The algorithm applied here mainly differs in the treatment of the background and in the segmentation routine. The background of each frame is removed by subtracting the strongly Gaussian-filtered frame from the original frame. The FHWM of the Gaussian typically is approximately four times the FWHM of the detection PSF. Hereafter, all regions with three connected (8-connectivity) pixels above threshold are registered and the respective bounding boxes are listed. The Gaussian mask-fitting algorithm described above is applied on them with a maximum of 100 centroid iterations, yielding a list with coordinate vectors $\vec{r}_{i_{1}}=\left(x_{i_{1}}, y_{i_{1}}\right)$ with $i_{1}=1 \ldots P_{1}$ for every registered event of channel 1 and $\vec{r}_{i_{2}}=\left(x_{i_{2}}, y_{i_{2}}\right)$ with $i_{2}=1 \ldots P_{2}$ for every registered event of channel 2 . In order to assign two registered events to the very same emitter, a transformation function between the coordinates of the two channels is needed:

$$
\vec{r}^{\prime}=f(\vec{r})=A \vec{r}+T,
$$

where $\vec{r}^{\prime}$ denotes the transformed coordinate vector, $A$ denotes an affine transformation and $T$ a translation vector. The transformation function $f(\vec{r})$ accounts not only for the 
(a)

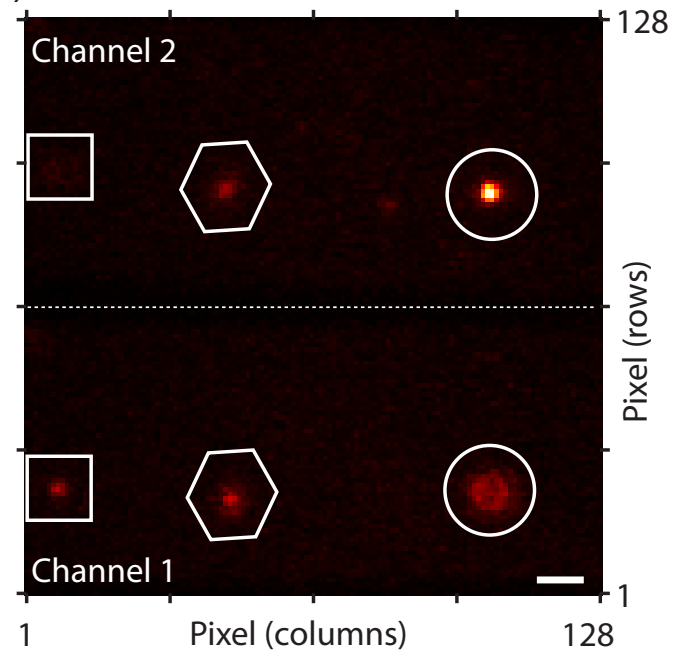

(b)

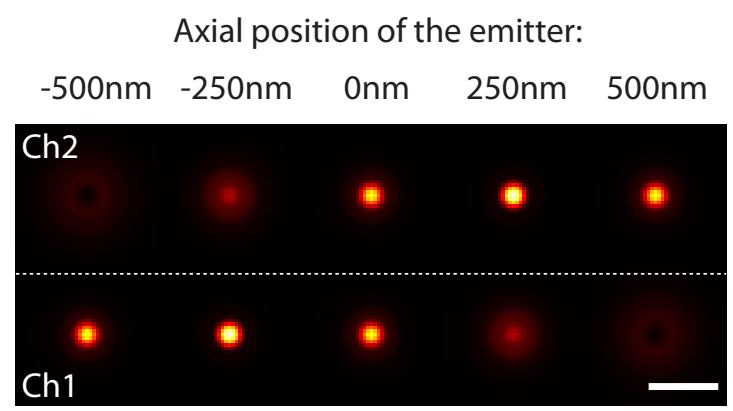

Figure 4.10: (a) Exemplary frame of a 3D recording of synthetic fluorophores. The sample is similar to the one in figure 4.16. The fluorescent signal was split into two parts of equal intensity. Each of the two is imaged onto one half of the EMCCD chip. The pixel rows 1...64 are assigned to channel 1 and pixel rows $65 \ldots 128$ to channel 2. The respective object planes are offset by $500 \mathrm{~nm}$. The white dotted line marks the separation between the two channels. For illustration purposes, three pairs of signal distributions are marked such that the same shapes indicate that the events stem from the same emitter. The emitter marked by the hexagon appears equally blurred and almost equally bright in both channels, indicating that its axial position is approximately in the center of the two object planes. Differently, the emitters marked by the square and the circle are brighter and sharper in channel 1 and channel 2, respectively. This indicates that their positions are shifted into the direction of the respective plane. The white bar represents $1 \mu \mathrm{m}$. (b) Theoretical PSFs (fluorescence wavelength $\lambda_{f l}=575 \mathrm{~nm}$, numerical aperture $\mathrm{NA}=1.3$, refractive index $\mathrm{n}=1.518$ ) which are centered at the axial positions indicated. The focal planes sit at $-250 \mathrm{~nm}$ and $250 \mathrm{~nm}$. The white bar represents $1 \mu \mathrm{m}$.

shift and scaling but also for shearing and for rotation of the second channel with respect to the first channel. The affine transformation $A$ is fully determined by six parameters, thus three different pairs of coordinate vectors are already sufficient for its determination. If more positions are present, $f(\vec{r})$ is found by a least-squares analysis. In practice, the mapping function can either be derived from calibration measurements with bright fluorescent beads whose axial position is such that their images appear equally blurred in both channels. Alternatively, the data of an actual SMS measurement can be used. To this end, events whose photon number exceeds a certain threshold and which are detected in both channels serve as the calibration data. The latter option retains the advantage that there is no time lag between the recording of the calibration data and the actual measurement in which a potential drift of the channels with respect to each other may occur. On the other hand, if there are inhomogeneities in the lateral distribution of the data subset which is used for calibration purposes, this might result in a non-uniform mapping accuracy over 
the field of view. Two events are assigned to the very emitter, if $\left|\vec{r}_{i_{1}}-f\left(\vec{r}_{i_{2}}\right)\right|<d$, where $d$ is typically 0.7 times the FWHM of the detection PSF.

Subsequently, the Gaussian weighted sums $b_{i_{1}}$ and $b_{i_{2}}$ are calculated, meaning that the raw data is multiplied by a Gaussian centered at positions $\vec{r}_{i_{1}}$ and $\vec{r}_{i_{2}}$, respectively. The FWHM of the Gaussian is matched to the FWHM of the detection PSF. If an event does not have a counterpart in the other channel, indicating that it is below threshold, the transformation function gives the partner's expected position. This position then either determines the center position for the Gaussian weighted sum or it serves as the starting point for an additional fixed-point iteration and a subsequent calculation of the Gaussian weighted sum. The final position $\vec{r}$ of an event is calculated from the weighted mean of the two paired coordinate vectors $\vec{r}_{1}$ and $\vec{r}_{2}$ :

$$
\vec{r}=\frac{b_{1} \vec{r}_{1}+b_{2} \cdot f\left(\vec{r}_{2}\right)}{b_{1}+b_{2}} .
$$

In case that the image of an event is in focus in channel $j$, the number of photons $N$ which are recorded for this event can be easily deduced from the Gaussian weighted sum via $N=2 \cdot c \cdot b_{j}$, where $c$ denotes the product of two correction factors. First, the image, which is the detection PSF, is multiplied by a Gaussian with the same FWHM. This results in a correction factor of approximately 2.4. Second, a correction factor which converts the camera counts into the number of photons is employed.

Obviously, the Gaussian weighted sum underestimates the photon number if the event is not in focus. This is because its fixed width does not account for the $\mathrm{z}$ dependence of the width of the fluorescent spot. Nevertheless, the Gaussian weighted sum constitutes an excellent and first hand mean for axial positioning which is not subject to moderate bleaching during the calibration measurement (cf. section 4.4.3).

In order to determine the photon number distribution of the registered events in an actual 3D SMS measurement, only those events are taken into account whose axial position coincide with one of the two focal planes within limits of $\pm 100 \mathrm{~nm}$. Within this range, there is no significant broadening of the detection PSF which would reduce the apparent photon number. This restriction usually does not introduce any systematic errors because scanning over the sample, which is done in all following measurements, ensures quasihomogeneous coverage of the whole sample region.

\subsubsection{D positioning algorithm}

Different degrees of blurring of the events shown in figure 4.10 (a) are easily perceivable. They hint at the respective emitter's axial position with respect to the image plane. If the two images of a particular emitter are similarly defocused, the emitter sits right in the center of the two object planes. If the blurring is less pronounced in one channel, the emitter's position is shifted from the center position in direction of the corresponding object plane. Figure 4.10 (b) illustrates the xy images of theoretical PSFs which are centered at the axial positions $-500,-250,0,250,500 \mathrm{~nm}$, where the objective planes sit at $-250 \mathrm{~nm}$ and $+250 \mathrm{~nm}$, respectively. The parameters for the PSF calculation were 

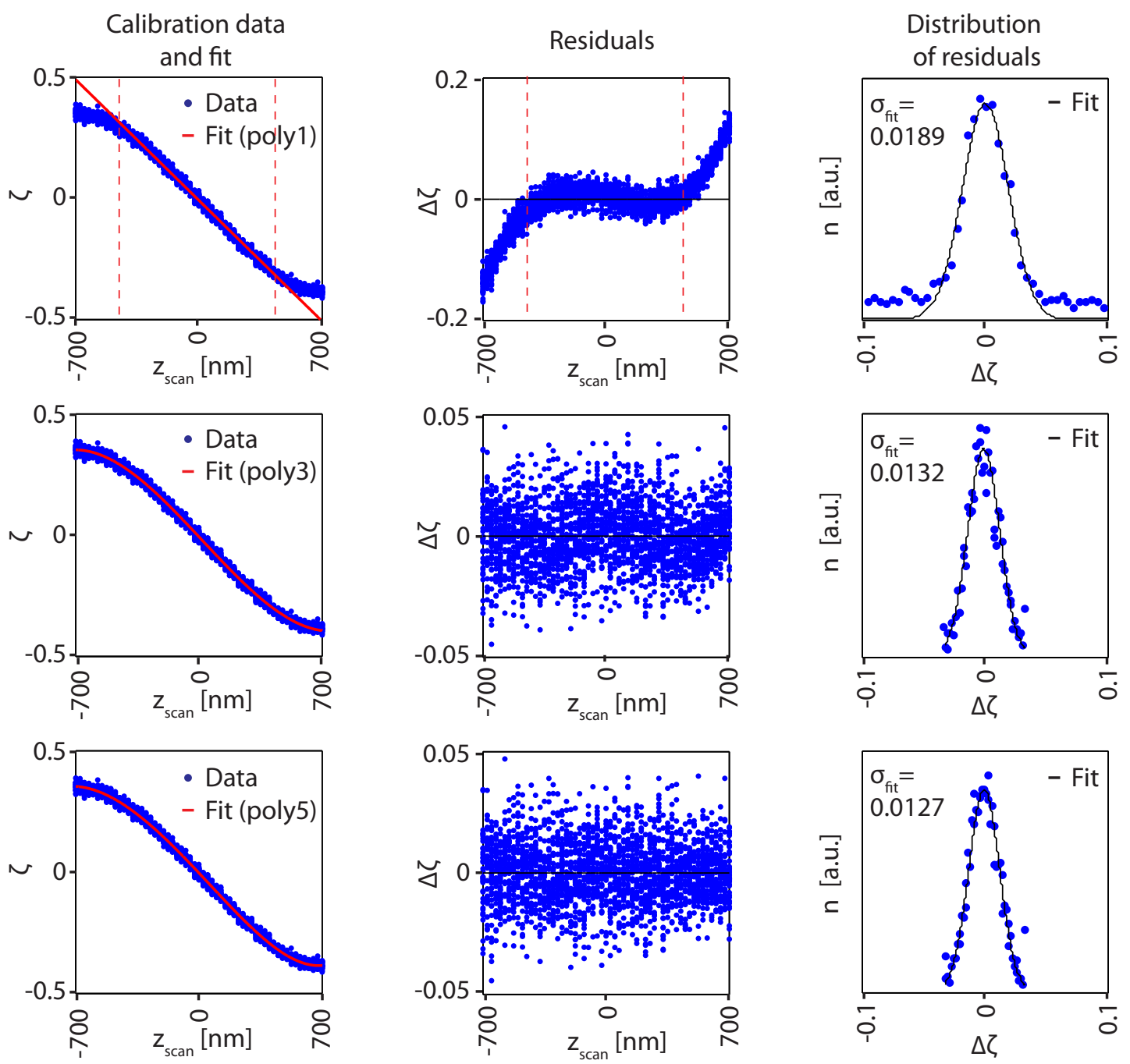

Figure 4.11: Calibration of the axial position with a fluorescent bead (diameter $200 \mathrm{~nm}$ ). The first column plots the contrast $\zeta$ (cf. equation (4.5)) against the axial position $\mathrm{z}_{\text {scan }}$ (blue dots) and shows the polynomial fits (red) of first (first row), third (second row) and fifth order (third row). The vertical red dashed lines represent the margins of the fit range (top row). The residuals of the data and the respective fit is shown in the second column. While the linear regression only fits the center part satisfactorily, the polynomial fit of third order is appropriate for the complete $\mathrm{z}$ range. However, its residuals still show slight oscillations which disappear when applying a polynomial fit of fifth order. The third column depicts the distribution of the residuals and their Gaussian fit whose standard deviation decreases for increasing fit order. 
chosen such that they match with the parameters of the experimental implementation. Note that a distinct pair of images in channel 1 and channel 2 can be assigned to each axial position of the PSF.

For precise axial positioning, a calibration measurement is indispensable. To this end, a fluorescent bead (FluoSpheres carboxylate-modified microspheres, $0.2 \mu \mathrm{m}$, red fluorescent, Invitrogen, Carlsbad, CA, USA) was axially scanned and typically 40 images were recorded at each step of $25 \mathrm{~nm}$. The distance of the observance planes was $500 \mathrm{~nm}$. This offset is a compromise between a rather homogeneous axial position error over a broad range and a lateral positioning accuracy which generally worsens for increasing distance [68]. Each frame was analyzed as described in section 4.4 .2 yielding the lateral position of the emitter, the respective scan position $z_{\text {scan }}$ and a list of the Gaussian weighted intensities $b_{1}\left(z_{\text {scan }}\right)$ and $b_{2}\left(z_{\text {scan }}\right)$ for channel 1 and 2 . By defining a contrast

$$
\zeta=\frac{1}{2} \cdot \frac{b_{1}-b_{2}}{b_{1}+b_{2}}
$$

the axial positions $z_{\text {scan }}$ are mapped to the range [-0.5...0.5]. Figure 4.11 presents a plot of $\zeta$ against $z_{\text {scan }}$ (blue dots) in the first column and investigates the quality of three fit functions (red line) by its residuals (second and third column). The linear regression (first row) was performed only for the data within the central axial range $-500 \mathrm{~nm}$ to $+500 \mathrm{~nm}$, while all data was used for the polynomial fit of third order (second row) and fifth order (third row). The residuals displayed in the second column show that the polynomial fits of third and fifth order are appropriate for the complete z-range as opposed to the linear regression. However, the residuals of the third-order fit still exhibit slight oscillations, which are not visible in the fifth-order fit. The distribution of the residuals along with the standard deviation of a Gaussian fit support these findings (third column). Since polynomial fits of higher order do not improve the fit quality (data not shown), the polynomial fit of fifth order is used in the following.

For investigating the capability of the method for axial positioning, two small fluorescent beads (diameter $40 \mathrm{~nm}$ and $100 \mathrm{~nm}$ ) were axially scanned over a range from $-600 \mathrm{~nm}$ to $+600 \mathrm{~nm}$. The back projected focal planes corresponded to planes in the sample at the axial positions $-250 \mathrm{~nm}$ and $+250 \mathrm{~nm}$, respectively. After each set of 50 (100) frames, the axial position was incremented by $50 \mathrm{~nm}$. The number of detected photons per frame was in the range of typical photon numbers of the fluorophores used in the following (cf. figure 4.16) for one bead and approximately nine times higher for the other bead. The data was analyzed in the same way as a regular 3D measurement would have been, here using the calibration bead shown in figure 4.11. For both fluorescent beads, the estimated axial positions (black dots) are plotted together with the scan position (red line), which was derived from the input voltage of the closed-loop nano-positioning device, in the first row of figure 4.12 (a) and (e). The fit positions from each set of frames, which was recorded at a constant axial scan position $\mathrm{z}_{\text {scan }}$, were further analyzed in the second and third row. Figure 4.12 (b) and (f) depict the deviations of the mean from the scan position for each set of frames. Although individual sets show a slight offset of their mean with respect 
Ca. 14200 photons per frame

(a)

(b)
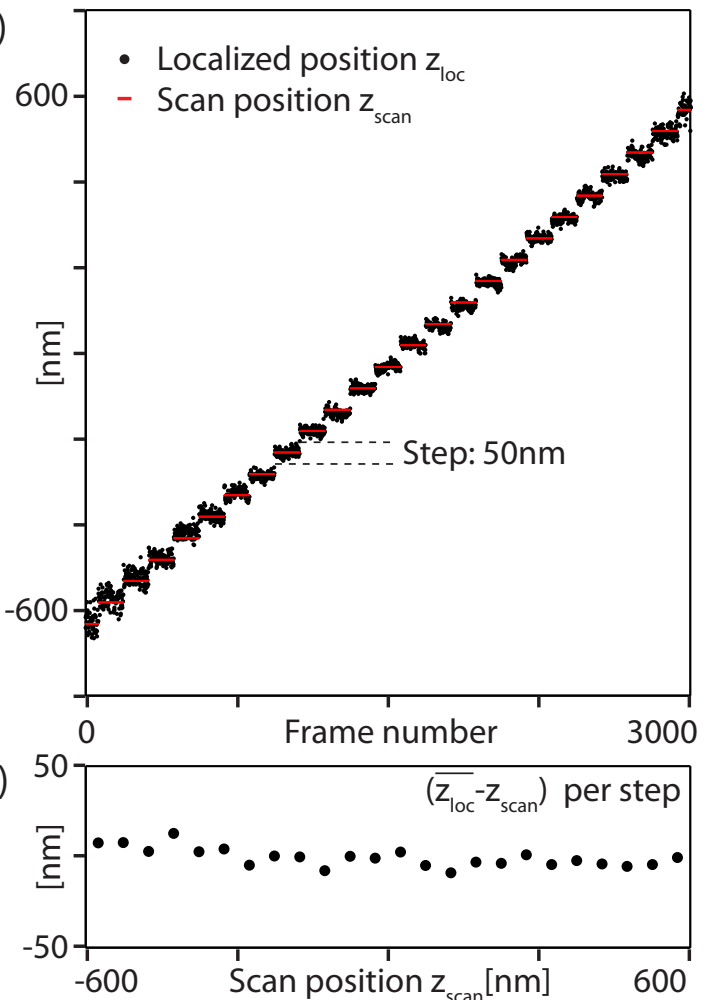

(c)

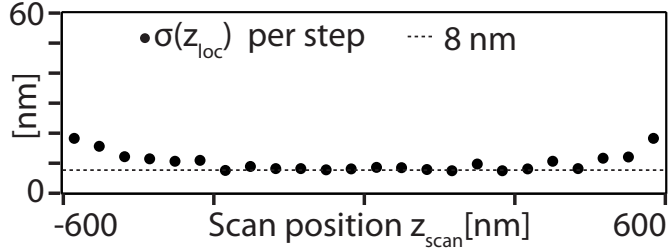

(d)

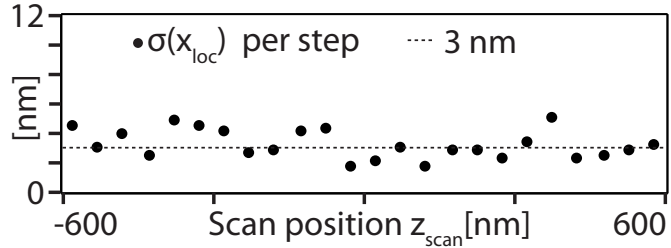

Ca. 1600 photons per frame

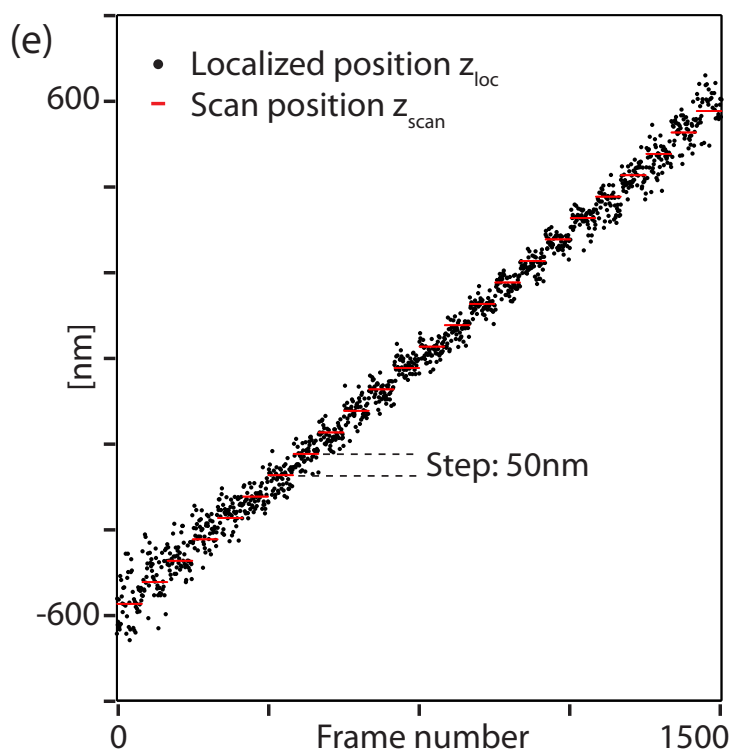

(f)

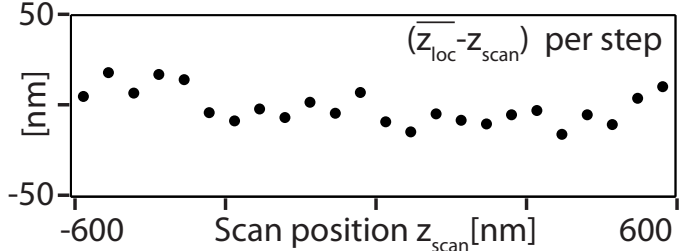

(g)
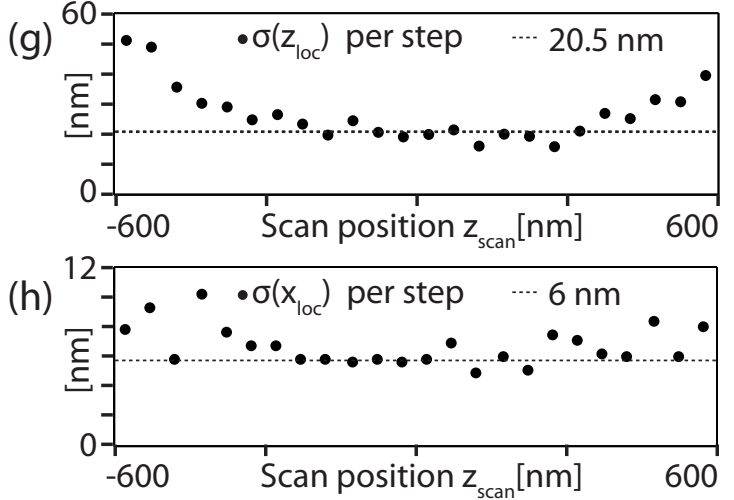

Figure 4.12: Positioning of fluorescent beads with detected photon number per frame in the range of a typical synthetic fluorophore (right column) and approximately nine times higher count (left column). Two beads ( $\mathrm{d}=100 \mathrm{~nm}$ (left) and $\mathrm{d}=40 \mathrm{~nm}$ (right)) were scanned over an axial range of $1200 \mathrm{~nm}$ m in steps of $50 \mathrm{~nm}$. At each step a set of 100 (left) or 50 (right) frames was taken. (a) and (e) depict the axial position $\mathrm{z}_{\text {scan }}$ (red line), as derived from the scanning device, and the position estimates $\mathrm{z}_{\mathrm{loc}}$ of the analysis routine (black dots). The difference between $\mathrm{z}_{\text {scan }}$ and the mean of $z_{\text {loc }}$ is shown for each set of frames in the second row, while the third row presents the standard deviation of $\mathrm{z}_{\mathrm{loc}}$ for each set. Note the high linearity of the mean estimated axial position and $\mathrm{z}_{\mathrm{scan}}$ over the full axial range. The mean of the standard deviation in the central axial region from -300 to $+300 \mathrm{~nm}$ scales in good approximation with the square root of the detected photon number. For comparison, the standard deviations in the $\mathrm{x}$ direction are shown in (d) and (h). 
to the scan position, a high degree of linearity is maintained over the full axial range of $1200 \mathrm{~nm}$. The offset can be explained by the fact that not the actual position is known, but only the input voltage of the scanning device. The positioning range of $1200 \mathrm{~nm}$ is fully compatible with the axial range of a $2 \mathrm{P}$ switching process $(\leq 1 \mu \mathrm{m})$ which permits 3D imaging of optical slices in thick media at once and without need for axially scanning. The third row presents the standard deviation of each set of frames, which attains its best values in an extended central axial region covering at least the axial distance of the focal planes. The standard deviations increase at the outer regions, which is expected by the shape of the calibration curve in figure 4.11. The mean of the standard deviation is approximately $8 \mathrm{~nm}$ for the brighter bead (ca. 14,200 detected photons) (cf. panel (c)) and approximately $20.5 \mathrm{~nm}$ for the darker bead (ca. 1600 detected photons) (cf. panel (g)) in the central region from -300 to $+300 \mathrm{~nm}$. The obtained axial localization accuracy is therefore 30-fold and 12-fold, respectively, better than the axial resolution of a conventional confocal microscope. The comparison of the two measurements shows that a 9-fold increase of the photon number leads to a 2.6-fold better axial localization accuracy. This agrees with the finding that the positioning accuracy in the axial dimension scales in good approximation with the inverse of the square root of the number of photons which was predicted by Chao et al. [90] and affirmed by Mlodzianoski et al. [89]. For comparison, the standard deviation of each set of frames for positioning in the x direction is shown in the fourth row. The mean of the standard deviation is approximately $3 \mathrm{~nm}$ (panel (d)) and approximately $6 \mathrm{~nm}$ (panel (h)) in the central region from -300 to $+300 \mathrm{~nm}$, which is 31 fold and 16-fold, respectively, better than the confocal resolution. The lateral positioning accuracy for the brighter bead is slightly worse than expected which hints at the fact that the setup experiences temporal lateral instabilities in the range of few nanometers.

In this section, the capability of the experimental platform to image volumes of $1200 \mathrm{~nm}$ thickness without axially scanning was proven. This means that the 2D SMS imaging protocol can be easily adopted. However, since the standard deviation increases slightly when moving away from the center, scanning is advisable if a uniform accuracy over the whole depth is favored.

\subsubsection{Experimental results for 3D imaging}

In order to demonstrate the feasibility of 3D SMS microscopy in 3D samples, surfacestained silica microspheres with a diameter of $500 \mathrm{~nm}$ were imaged. To this end, aminofunctional silica microspheres (500 nm diameter, PolySciences Europe GmbH, Eppelheim, Germany) were surface-stained with the photochromic fluorescent compound SRA577 (switchable rhodamine amide with emission maximum at $577 \mathrm{~nm}$ ) [80] which is a derivative of rhodamine B. The actual sample was prepared as follows. Cover slips were rinsed with deionized water for $15 \mathrm{~min}$ and cleaned in a low pressure plasma system (Femto$R F$, Diener Electronic, Nagold, Germany) to annihilate any fluorescent background on the glass surface. Before adhering the surface-stained beads with poly-L-lysine (SigmaAldrich Chemie GmbH, Taufkirchen, Germany) to the surface (cf. section 3.3), the cover slip was incubated with unstained silica particles suspended in $0.1 \%(\mathrm{w} / \mathrm{v})$ PVA $(235 \mathrm{~nm}$ 
diameter $\mathrm{SiO}_{2}$-microparticles, Microparticles $\mathrm{GmbH}$, Berlin, Germany) in water until the solvent had evaporated. This formed a rough surface, which established contact sites with the $500 \mathrm{~nm}$ microspheres at positions which are very likely different from the foot of the beads. Quenching effects because of the close contact between the two silica surfaces are thus avoided at this position. Subsequently, the sample was embedded in standard Mowiol, whose $\mathrm{pH}$ was adjusted to 10.0 using a carbonate-bicarbonate buffering system.

A wide-field illumination with $375 \mathrm{~nm}$ light was used for switching the compound SRA577. The use of the $2 \mathrm{P}$ switching process did not have any advantages compared to the $1 \mathrm{P}$ switching process because the sample's axial extent was less than that of the $2 \mathrm{P}$ PSF. The $532 \mathrm{~nm}$ laser line was applied for wide-field excitation with an average power of $20 \mathrm{~kW} / \mathrm{cm}^{2}$. The EMCCD camera was running at a frame rate of $100 \mathrm{~Hz}$. As illustrated in figure 4.12, axial positioning over a range of approximately $1200 \mathrm{~nm}$ is feasible without scanning. However, the positioning accuracy depends on the axial position of the marker. In order to maintain a homogeneous accuracy over the full range, the sample was moved axially in steps of $25 \mathrm{~nm}$ after every 200th frame over a full range of $500 \mathrm{~nm}$. In order to minimize the effects of potential sample drift and to ensure detection of equal percentages of the emitter distribution over the complete axial imaging range, the sample was not scanned once, but the scanning was done repeatedly for 156 times which resulted in the recording of 780,000 frames. For densely stained samples, the density of initially switched on dye molecules is high enough so that no additional activation with the UV light is necessary. Conversely, it may even be that the initial event density is too high so that pre-bleaching is necessary. As the percentage of spontaneously switched on SRA577 molecules can be tuned with the $\mathrm{pH}$ within limits [91], the choice of Mowiol with $\mathrm{pH}$ 10.0 ensured an adequate density of events at the beginning of the recording. When the event density was below optimum, the power of the UV light was increased stepwise up to $18 \mathrm{~W} / \mathrm{cm}^{2}$, hereby tuning the density to a reasonable level. Figure 4.13 (a) shows an $\mathrm{xz}$ slice through the center of a $500 \mathrm{~nm}$ microsphere in a $50 \mathrm{~nm}$-'smoothed histogram' view. Approximately 1000 events contributed to the image. Their photon distribution per event can be fitted well with an exponential decay function with an expectation value of 700 photons. Note that the expected positioning accuracy cannot be directly derived from the bead measurements presented in figure 4.12. The reason for this is that the photons per event follow a Poisson distribution for the fluorescent bead, whereas the photon number per event can be described by a geometrical distribution in the present measurement. Thus, the distribution of localized positions at the mean photon number $(\bar{N})$ experiences admixtures of broader and more probable distributions (for $N<\bar{N}$ ) and of narrower, but less likely ones (for $N>\bar{N}$ ). The FWHM of the effective distribution depends on $\bar{N}$, the photon threshold, the FWHM of the diffraction-limited detection PSF and the photon distribution [68]. The voxel size is $25 \mathrm{~nm}$ in the $\mathrm{x}$ and $\mathrm{z}$ direction and $100 \mathrm{~nm}$ in the y direction. For comparison, a confocal image of a microsphere of the same charge, which was surface-stained with Rhodamine B, was taken (SP5, Leica Microsystems, Wetzlar, Germany). It was deconvolved with the Huygens deconvolution software (Scientific Volume Imaging, Hilversum, the Netherlands) by applying an experimentally determined PSF. Figure 4.13 (b) depicts the deconvolved confocal data of the reference microsphere. 

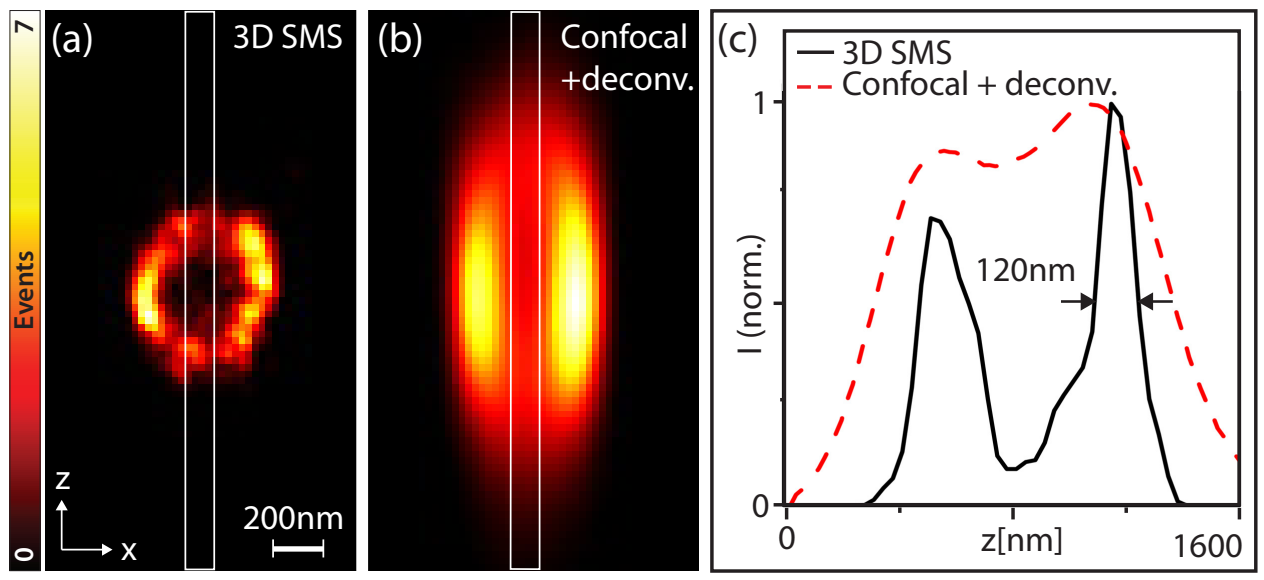

Figure 4.13: 3D SMS imaging of a $500 \mathrm{~nm}$ surface-stained silica microsphere. (a) shows the $50 \mathrm{~nm}$-'smoothed histogram' representation of the slice running through the center of the sphere. Approximately 1000 events contributed to the image. The voxel size is $25 \mathrm{~nm}$ in the $\mathrm{x}$ and $\mathrm{z}$ direction and $100 \mathrm{~nm}$ in the y direction. For comparison, (b) presents the deconvolved confocal image of a similar bead of the same charge. The upper and the lower surface of the microsphere cannot be clearly distinguished as opposed to the 3D SMS image. The direct comparison of the $\mathrm{z}$ profiles highlights the gain in axial resolution.

The $\mathrm{z}$ profiles integrated over the area indicated by the white rectangles in panel (a) and (b) illustrate the resolution enhancement. Whereas the upper and the lower surface cannot clearly be distinguished from each other in the deconvolved confocal image (dashed red line), they are well separated in the 3D SMS image (black line).

Having successfully illustrated the 3D imaging capability of thin specimens in the preceding paragraph, the method was applied to a thicker sample whose axial extent is well above the positioning range of the microscope (cf. section 4.4.3) and well above the axial FWHM of a 2P PSF. Therefore, it is worthwhile to exploit the advantages of switching by a $2 \mathrm{P}$ process [38][40]. Activation of the compound SRA577 cannot only be induced by UV light, but also by the simultaneous absorption of two photons in the red or infrared range [80]. The light intensity must be very high to facilitate the switching process which is the reason why the light of a pulsed laser system was focused into the sample as described in section 4.4.2. Since the probability of 2P activation scales with the square of the intensity, the switching process is effectively confined to the vicinity of the focal plane. This means that only those molecules residing in this restricted volume (typically $\leq 1 \mu \mathrm{m}$ in the axial direction) undergo the imaging and bleaching process, whereas other ones remain in their off-state. On the one hand, this implies that the molecules unaffected by the $2 \mathrm{P}$ switching light do not contribute to any perturbing background fluorescence which is especially important for densely stained samples. On the other hand, these molecules are saved from unnecessary bleaching and stay available for later read out, which retains a great advantage especially when sparsely stained samples are imaged. However, appli- 

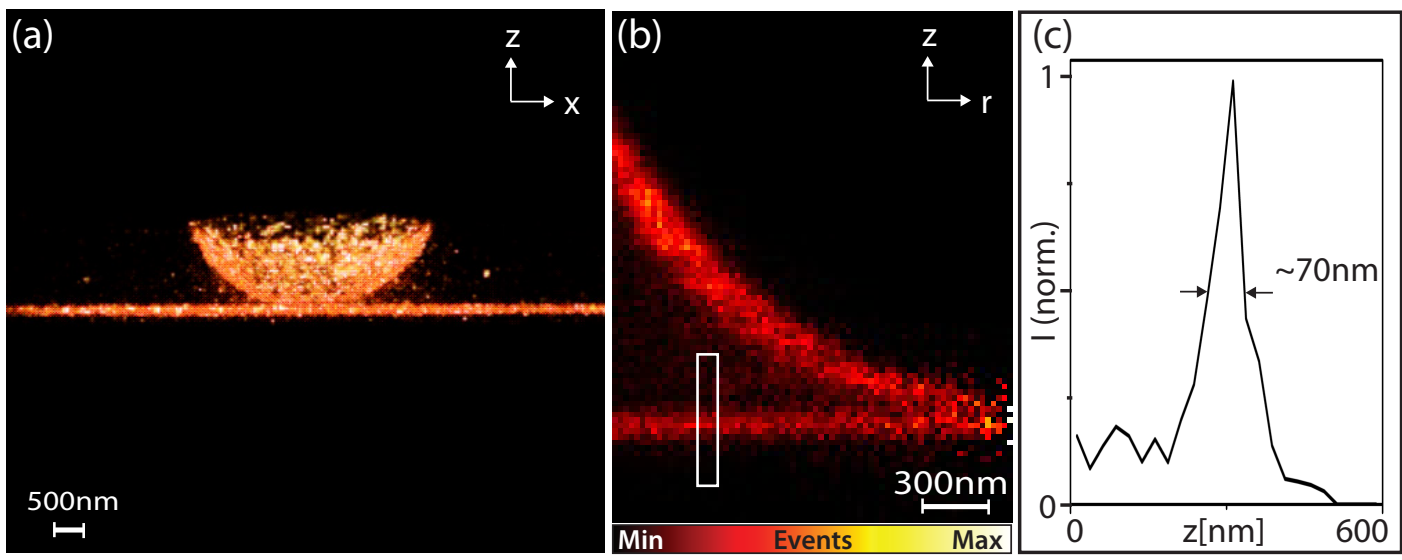

Figure 4.14: 3D SMS imaging of an axially extended sample. An amino-modified silica microsphere (diameter $5 \mu \mathrm{m}$ ), which is surface-stained with SRA577, sits on a monolayer of the same dye. This is visualized by the surface-rendered 3D reconstruction in panel (a). The normalized radial profile in (b) is presented in a 'histogram' view with a pixel size of $25 \mathrm{~nm}$. (c) The axial positioning accuracy in the SMS image is conservatively estimated from the axial profile integrated over the region marked in (b).

cation of $2 \mathrm{P}$ switching light was found to induce stronger bleaching of the fluorophores than $1 \mathrm{P}$ switching light. The application of $2 \mathrm{P}$ switching light, nevertheless, presents a way for sectioning by optical means which facilitates the efficient and background-free imaging of single molecules events, that are localized in three dimensions later-on. It should be mentioned that the excitation illumination was still performed in a wide-field configuration.

A monolayer of compound SRA577 was prepared by incubating a silanized cover slip with SRA577-NHS-ester. Analogous to the treatment of the $500 \mathrm{~nm}$ microspheres, aminomodified silica microspheres (diameter $5 \mu \mathrm{m}$ ) were surface-labeled with SRA577 and directly attached to cover slips with poly-L-lysine. The sample was embedded in standard Mowiol ( $\mathrm{pH}$ 9.5). It was imaged with a frame rate of $200 \mathrm{~Hz}$ for 460,000 frames which amounted to a total acquisition time of $38 \mathrm{~min}$. Axial scanning was performed repeatedly in steps of $200 \mathrm{~nm}$ over a range of $2 \mu \mathrm{m}$. The excitation intensity was kept constant at an average power of $13 \mathrm{~kW} / \mathrm{cm}^{2}$, whereas the light intensity of the $2 \mathrm{P}$ light $(740 \mathrm{~nm})$ was increased stepwise from zero up to approximately $2.5 \mathrm{MW} / \mathrm{cm}^{2}$ in one single focus. Six foci were simultaneously scanned over the sample, considering that the complete field of view was covered approximately twice during one camera exposure time of $5 \mathrm{~ms}$. In order to get a better notion of the sample layout, a surface-rendered 3D reconstruction of the 3D SMS data is depicted in figure 4.14 (a) to which approximately 44,000 events contributed. A radial profile centered at the center of mass of the spherical shell illustrates the clear separation of the microsphere surface and the monolayer in figure 4.14 (b). The averaged profile over an area indicated by the white rectangle in panel (b) serves as a conservative estimate for the localization accuracy in this image because any tilt, curvature or rough- 


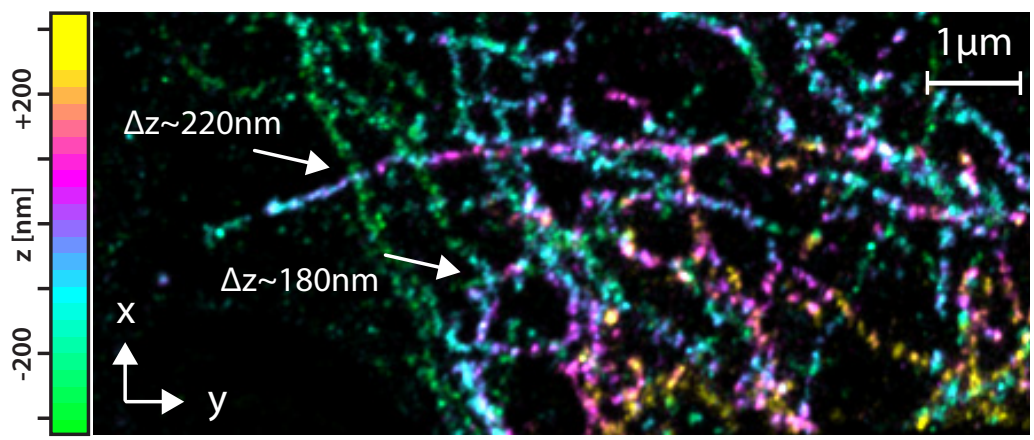

Figure 4.15: 3D SMS imaging of the microtubular network in a whole mammalian cell. The projection of the $50 \mathrm{~nm}$-'smoothed histogram' view is color-coded for the axial position as indicated by the colorbar. The filaments at the marked intersections have an axial separation of $180 \mathrm{~nm}$ and $220 \mathrm{~nm}$.

ness enlarges the apparent thickness of the monolayer (panel (c)).

The benefits of 3D resolution enhancement are not limited to technical samples. Here, the applicability of the experimental platform to the imaging of biological samples is demonstrated. The microtubular network ( $\beta$-tubulin) of fixed whole mammalian PtK2 cells was immuno-stained with SRA577. Please refer to the appendix for details on cell culture and immuno-staining. The SMS data was recorded at $100 \mathrm{~Hz}$ at a constant excitation intensity $(532 \mathrm{~nm})$ of $13 \mathrm{~kW} / \mathrm{cm}^{2}$ and a gradually increasing intensity of the $1 \mathrm{P}$ switching light $(375 \mathrm{~nm})$. Sets of 200 frames were taken at every axial step of $25 \mathrm{~nm}$ and the total scan range of $500 \mathrm{~nm}$ was covered 40 times. Over 100,000 events contribute to the projection of a $50 \mathrm{~nm}$-'smoothed histogram' view, in which the color codes for the axial position as indicated by the color bar (cf. figure 4.15). This image not only shows considerable improvement in the lateral resolution compared to the wide-field fluorescence imaging mode, but also provides the axial information that is not available in the conventional mode. Here, multiple layers of filaments are clearly visible. In the intersections marked by the white arrows, the tubules which are crossing in the xy projection are axially separated by approximately $180 \mathrm{~nm}$ and $220 \mathrm{~nm}$, respectively.

\subsection{Applications of 3D SMS microscopy}

The experimental results presented in section 4.4.4 prove the feasibility of 3D SMS microscopy for imaging of thin and thick technical and biological samples. It is therefore worthwhile to utilize this imaging method in the context of interesting fields of applications for which two examples will be given in this section. 


\subsubsection{Structural analysis of colloidal crystals}

The field of colloidal physics has experienced large sophistication in the last two decades which is accompanied by an unparalleled revolution in particle synthesis [92]. Colloidal science studies systems in which one material ("dispersed phase") is evenly dispersed in a second material ("continuous phase"), where the first material ("colloids") is neither truly dissolved nor suspended. The diameter of the colloids typically range from one nanometer to one micrometer. The ability to easily tune free parameters like size, shape and composition has led to a high popularity of colloidal materials as models for atomic and molecular systems. Studies of a variety of phenomena have been conducted including the glass transition [93], phase transitions such as melting [94] and the process of selfassembly [95]. Structural information with length-scales of interest has typically been obtained by scattering-based techniques, electron or scanning probe imaging. However, the 3D morphology is of great interest in many studies. Hence, confocal fluorescence microscopy has emerged as a powerful tool, which provides $3 \mathrm{D}$, non-invasive and dynamic information [96] [97]. The limited resolution of a confocal microscope especially in the axial direction however restricts the minimum size of the particles to about one wavelength of the applied excitation light. This constraint has severe implications on the variety of experiments which can be conducted because the size of the particles constitutes an important parameter. For non-interacting, hard spheres, which are exposed to excluded volume interaction and entropic forces and which show Brownian motion, the size influences sedimentation and overall dynamics. For colloids with more complex interactions and shapes, the size and orientation of the particles affect the long-range forces which govern the process of self-organization. Hence, the study of colloidal systems will benefit highly from the advent of far-field imaging techniques with high resolution in three dimensions. Recently, 3D imaging of densely packed colloidal nanostructures using the complementary high-resolution far-field STED technique has been demonstrated [98]. The resolution enhancement to up to $43 \mathrm{~nm}$ in the lateral and $125 \mathrm{~nm}$ in the axial direction has been shown.

In order to exemplify the applicability of 3D SMS microscopy to the study of the 3D morphology of colloidal material, fluorescent core-shell silica nanoparticles were prepared as described by Foelling et al. [99]. The cores with a diameter of approximately $80 \mathrm{~nm}$ were doped with the switchable rhodamine amide SRA545 [100], whereas the silica shell remained unlabeled. The total diameter of the particles was grown up to 180$190 \mathrm{~nm}$ to tune a desired separation between cores in an array of nanoparticles. The inset in figure 4.16 (a) illustrates the particles' layout and shows a scanning electron micrograph of the surface of a colloidal structure which was prepared similarly to the sample in the 3D SMS recording. For this, a small droplet of core-shell particles suspended in ethanol was placed on a cover slip which had been rinsed with acetone and dried in a flow of Nitrogen. After the ethanol had evaporated, two cover slips were placed on top of each other with the nanoparticles facing inwards. A small volume of a glycerol-water solution (refractive index $n=1.460$ ) between the cover slips acted as a spacer and served as embedding medium to mitigate the effect of scattering. The amount of medium was 

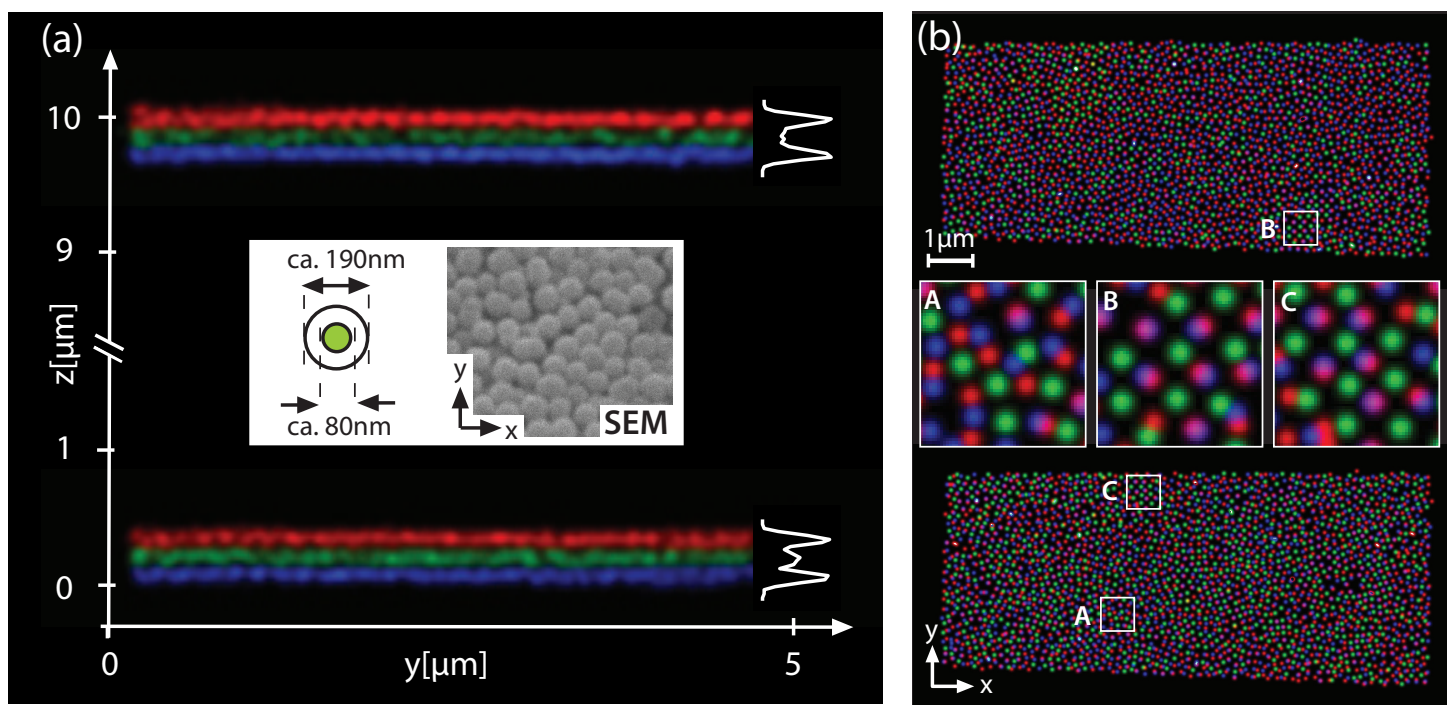

Figure 4.16: Analysis of colloidal nanostructures. The inset in (a) explains the core-shell particles' layout and shows a scanning electron micrograph (SEM) of the surface of a colloidal structure. (a) presents the xz projection of two sample regions close to the objective lens and at an axial depth of approximately $10 \mu \mathrm{m}$. In each, a stacking of three layers is visible. Every located sphere is visualized by a Gaussian with $80 \mathrm{~nm}$ FWHM as described in the text in more detail. The axial profiles (white line) of the raw SMS data over the full y axis show an axial distance between single layers of approximately $130 \mathrm{~nm}$. (b) The xy projections with approximately 3000 localized spheres in each region show regions with no apparent ordering (inset A) and regions with an ABA-layering system (insets B, C) as indicated by the mixed colors of the overlapping spheres.

chosen such that the two sample planes were separated by approximately $10 \mu \mathrm{m}$ in the axial direction. Hereby, a sample was prepared, which does not only demonstrate imaging colloidal self-assembly on the nanoscale, but it also serves as a test specimen for imaging objects which reside $10 \mu \mathrm{m}$ deep inside the sample.

The two sample regions were recorded sequentially due to limitations of the scan protocol imposed by the applied software. Both regions were imaged at a frame rate of $100 \mathrm{~Hz}$ for 220,000 and 240,000 frames, respectively, which were divided into sets of 4000 frames. The total scan range was $400 \mathrm{~nm}$ each, which was cut into steps of $25 \mathrm{~nm}$. Consequently, 250 frames were recorded at each of the 16 axial positions. The excitation power was kept at a constant level of $13 \mathrm{~kW} / \mathrm{cm}^{2}$, while the intensity of the $2 \mathrm{P}$ switching light $(740 \mathrm{~nm})$ was increased stepwise from zero to $2 \mathrm{MW} / \mathrm{cm}^{2}$ in one focal volume for the lower region and from $1 \mathrm{MW} / \mathrm{cm}^{2}$ to $3 \mathrm{MW} / \mathrm{cm}^{2}$ in one focus for the upper region. Interestingly, the single-exponential fits of the photon distributions of both regions feature the same expectation value (ca. 1500 photons per event), implying that the average resolution enhancement in the upper layer is not degraded with respect to the lower region by its absolute axial position in the sample.

In order to aid in the visualization of the morphology of the colloidal crystal, prior knowledge of the samples can be exploited. The 'histogram' view of the SMS data with 
a voxel size of $12.5 \mathrm{~nm}$ laterally and $25 \mathrm{~nm}$ axially was deconvolved with a 3D Gaussian function whose FWHM was matched to the diameter of the colored cores of the colloids. The detection of local intensity maxima identified the approximate position of each sphere which was subsequently localized more precisely by a center of mass analysis. The resulting grid of points was convolved with a 3D Gaussian with $80 \mathrm{~nm}$ FHWM. The Gaussians were colorized on the basis of the axial positions of their centers. Those Gaussians whose center positions fell into the five pixels, which are in the center of the respective layering system, were colored green, whereas the ones below were colored in blue and the ones above in red. The projection of all $\mathrm{xz}$ slices of this colorized 3D reconstruction of each of the two sample regions is presented in figure 4.16 (a). It becomes obvious that the core-shell particles are assembled in three layers in each of the two sample regions.

The distance between the outer peaks of the averaged axial intensity profile of the raw SMS data (white lines) is approximately $260 \mathrm{~nm}$ for each of the two regions, resulting in an average distance between two single layers of approximately $130 \mathrm{~nm}$. This value is smaller than the expected distance $z_{\text {pitch }}=\sqrt{2 / 3} \cdot d=151 \mathrm{~nm}$ for a closed-packed structure of spheres with the diameter $d=185 \mathrm{~nm}$. This indicates that the material's structure differs from a closed-packed one. The stacking sequence of the two regions is visualized in an xy projection for both regions in figure 4.16 (b) top and bottom. When assigning blue spheres to the bottom layer, green ones to the middle and red ones to the top layer, it becomes obvious that no overall order can be found (inset A). Still, in some small regions an ABA-layer sequence is clearly visible as illustrated by the insets $\mathrm{B}$ and $\mathrm{C}$ in panel (b). Overlapping spheres of the bottom and the top layer are here identified by mixed colors.

By means of 3D SMS microscopy the morphology of a colloidal material, which had been created by the self-organization of silica nanospheres during evaporation of the solvent, was visualized on a scale which is not accessible by confocal microscopy. Further, the localization could be done $10 \mu \mathrm{m}$ deep inside the sample without any signs of impairment of the resolution enhancement.

\subsubsection{In-vivo measurements: structural changes with time}

Far-field light microscopy has emerged as the most popular tool for imaging in the life sciences because of its key advantages such as non-invasive imaging and sensitive detection of macromolecules. While immuno-staining with antibodies and organic fluorophores is mostly applied to fixed cells, the present method of choice for tagging proteins of interest in living cells is genetic fusion which has been greatly assisted by the rapid extension of the GFP-family. Therefore, far-field light microscopy would be ideal for imaging the 3D morphology of living cells if a resolution enhancement in all three dimensions beyond the diffraction limit was feasible. This would allow studying processes and structures on the nanoscale. Several approaches in the study of living cells beyond the diffraction limit have been conducted. SMS-based approaches have largely been limited to two dimensions. An example is the study of adhesion dynamics in the plasma membrane by Shroff et al. [101]. The work presented by Vaziri et al. [82] however went a step further and introduced an additional optical sectioning by temporal focusing of light, resulting in a depth of field 

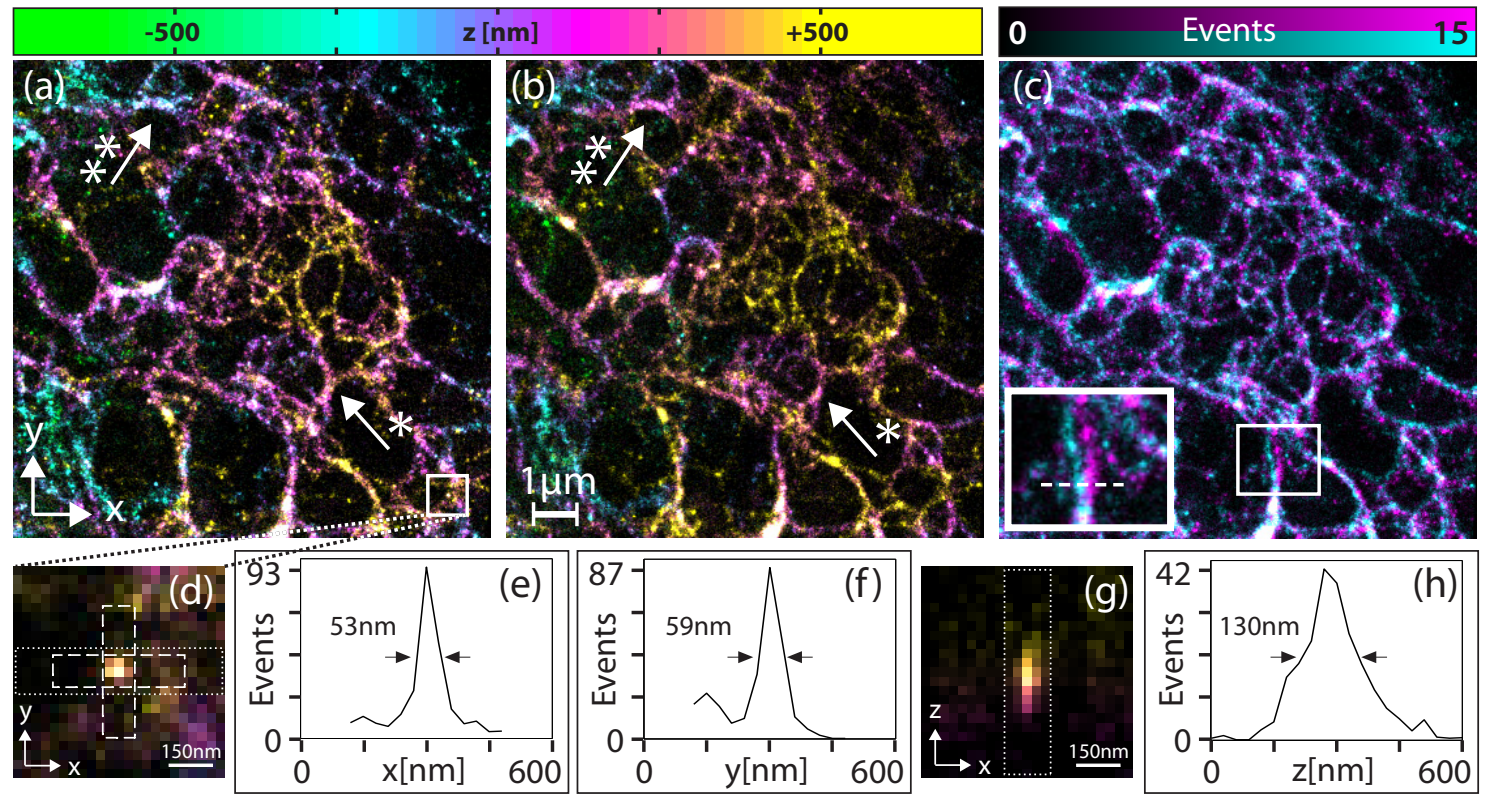

Figure 4.17: Keratin-network in a living PtK2 cell for which both 3D position information and time information is available. The projection of the $30 \mathrm{~nm}$-'smoothed histogram' view of the SMS data, which was recorded at two different points in time, is shown in (a) and (b). The recording time for each image was $9.33 \mathrm{~min}$ with a break of $20 \mathrm{~min}$ in between. The color-coding of the axial position reveals different axial movements of specific filamentous structures, in particular no change of the position (' $'$ ', lower arrow) in contrast to movement of approximately $200 \mathrm{~nm}$ ('**', upper arrow). (c) presents the overlay of (a) and (b), in which lateral movements become apparent. The inset enlarges the marked region (white rectangle) in which an individual filament has moved by approximately $100 \mathrm{~nm}$ along the white dashed line. Panel (d) is a close-up of the region marked in (a) by the white rectangle. The spatial dimensions of the object which is likely an agglomeration of proteins serve as an estimate for the positioning accuracy in this image. The lateral profiles integrated over the dashed rectangles marked in (d) have a FWHM below $60 \mathrm{~nm}(\mathrm{e}$, f). For estimating the axial precision, an xz projection of the dotted region marked in (d) is shown in panel (g) together with the axial profile (h) integrated over the dotted region in (e). Its FWHM is $130 \mathrm{~nm}$. 
of $1.9 \mu \mathrm{m}$. With the help of the complementary STED-technique, time lapse studies of a fluorescent protein tagged organelle in living cells have recently been presented, which revealed a 3-fold axial resolution improvement compared to confocal microscopy [102].

Here, 3D SMS imaging in living cells is presented, in particular the structural and temporal changes of the intermediate filament networks built up from vimentin and keratin6a in HeLa and PtK2 cells, respectively. These recordings are the first 3D SMS images of living cells.

Intermediate filaments are one of the three components of the cytoskeleton of mammalian cells. They come in a wide variety of types [103] and they provide mechanical strength, resistance to shear stress and are involved in a variety of other processes including distribution of cellular organelles [104]. Intermediate filaments have long been considered to be static. However, over the last decade it has been shown that they are highly dynamic with respect to the movement of the entire network [105]. For instance, vimentin and keratin filaments have been seen to bend and undergo wavelike motions [106] [107].

In order to tag the structures, the fusion proteins Vimentin-Dendra2 and Keratin6atdEosFP were utilized. EosFP is a photo-convertible fluorescent protein which was first described by Wiedenmann et al. in 2004 [108]. The protein originally stems from the scleractinian coral Lobophyllia hemprichii and emits strong green fluorescence $(516 \mathrm{~nm})$ which changes to red fluorescence $(581 \mathrm{~nm}$ ) upon illumination with near UV light (around $400 \mathrm{~nm}$ ). The monomeric protein Dendra2 is an improved version of Dendra derived from the octocoral Dendronephthya sp. [109]. It likewise shows green-to-red conversion induced by UV to violet or blue light. Its green and red emission maxima are at $505 \mathrm{~nm}$ and $558 \mathrm{~nm}$, respectively. The irreversible green-to-red photoconversion arises from a lightdriven covalent modification in both cases, in particular, it is associated with a cleavage of the peptide backbone [110] [111]. For details on DNA constructs, cell culture and transfection, please refer to the appendix. For imaging, the growth medium was exchanged with Dulbecco's modified eagle medium without phenol red. The cells were imaged at room temperature and did not show any signs of cell death during the measurements. The $560 \mathrm{~nm}$ laser line was used for excitation and $405 \mathrm{~nm}$ light for green-to-red conversion.

Figure 4.17 shows a part of the keratin network in a living PtK2 cell. In panels (a) and (b), the 3D information is presented as a projection of a $30 \mathrm{~nm}$-'smoothed histogram' view with a pixel size of $30 \mathrm{~nm}$. The photon distribution per event can be fitted well with an exponential decay function with an expectation value of 120 photons. The axial position is color-coded as indicated. Both images were obtained within $9.3 \mathrm{~min}$ each, in which 80,000 frames were taken while repeatedly axially scanning over the structure in 10 steps of $50 \mathrm{~nm}$. The time interval between the last frame of the first image and the first frame of the second one is $20 \mathrm{~min}$, in which the cell was neither exposed to the green excitation light $\left(5 \mathrm{~kW} / \mathrm{cm}^{2}\right)$ nor to the blue switching light $\left(15 \mathrm{~W} / \mathrm{cm}^{2}\right)$. A comparison of the colors at specific positions in (a) and (b) illustrates that individual filaments have moved during the waiting time of $20 \mathrm{~min}$. For exemplification, the upper arrow ('**') marks a filament which has moved up by approximately $200 \mathrm{~nm}$. In order to rule out an absolute axial drift of the cell, the lower arrow ('*') points at a filament, which remained at a constant 
axial position. The lateral changes over time become apparent in figure 4.17 (c) which is an overlay of (a) and (b). Here, the number of events is presented with two different color tables, each representing one of the two images. Again, there is no absolute shift of the whole region detectable, but movements and bendings in various directions, of which one is focused on in the inset. The lateral profile along the dashed white line reveals that the filament has moved by approximately $100 \mathrm{~nm}$ during the waiting time. In order to assess the spatial resolution, the object indicated in figure 4.17 (a) by the white rectangle is scrutinized which likely presents an agglomeration of several tdEosFP proteins. Panel (d) shows a close-up of the object. The lateral profiles in (e) and (f) which are integrated over the regions marked by the dashed rectangles in (d) reveal that the lateral positioning accuracy is better than $60 \mathrm{~nm}$. For comparison, the xz projection of the dotted region in (d) is shown in $(\mathrm{g})$. The axial profile integrated over the dotted region in $(\mathrm{g})$ discloses an axial positioning accuracy of at least $130 \mathrm{~nm}(\mathrm{~h})$. These values are conservative estimates for the localization accuracy since any movement or a non-negligible spatial extent of the object deteriorates the apparent resolution.

Information about the dynamics of a spatial modification over time can be retrieved by a series of images, which reveals not only the starting configuration and the end configuration, but also the speed and the continuity of the dynamics. Figure 4.18 (a) to (h) presents a time-series of a surface-rendered 3D reconstruction of the vimentin-network in a living HeLa cell. Note that the first panel (a) is repeated at the bottom right (i) which facilitates assessing the structural change over time. The surface rendering was performed on the $100 \mathrm{~nm}$-'smoothed histogram' view with a pixel size of $50 \mathrm{~nm}$ (Amira, Visage Imaging, Inc., San Diego, CA, USA). The total acquisition time for this measurement was $14.6 \mathrm{~min}$ in which almost 600,000 events were registered. The photon distribution per event can be fitted well with an exponential decay function with an expectation value of 175 photons. This means that the expected positioning accuracy is better than the one in the image of the keratin network in figure 4.17. For representation, events obtained within time spans of 4.9 min were combined in one image, while the time interval between the first frame of each image was $82 \mathrm{~s}$. By using this floating average, a certain dynamic range for each image is maintained as well as dynamic information of eight time intervals is gained. The excitation laser power was kept constant at $6.5 \mathrm{~kW} / \mathrm{cm}^{2}$, while the switching power was increased stepwise from zero to $3.5 \mathrm{~W} / \mathrm{cm}^{2}$. The data was recorded with a camera exposure time of $7 \mathrm{~ms}$ and 149 stacks (with 840 frames each) were taken with an axial step size of $50 \mathrm{~nm}$. Therefore, 12 frames were recorded at each of the 70 axial steps. Since the filamentous structure extends over the full axial scan range of $3.5 \mu \mathrm{m}$ and beyond, axial movement of bundles of filaments, which are near the borders of the detection range, leads to them leaving or entering the field of view. Nevertheless, structural changes of the network over time are vividly revealed like the bending and shifting of the two bundles of filaments which are focused on in the inset. 


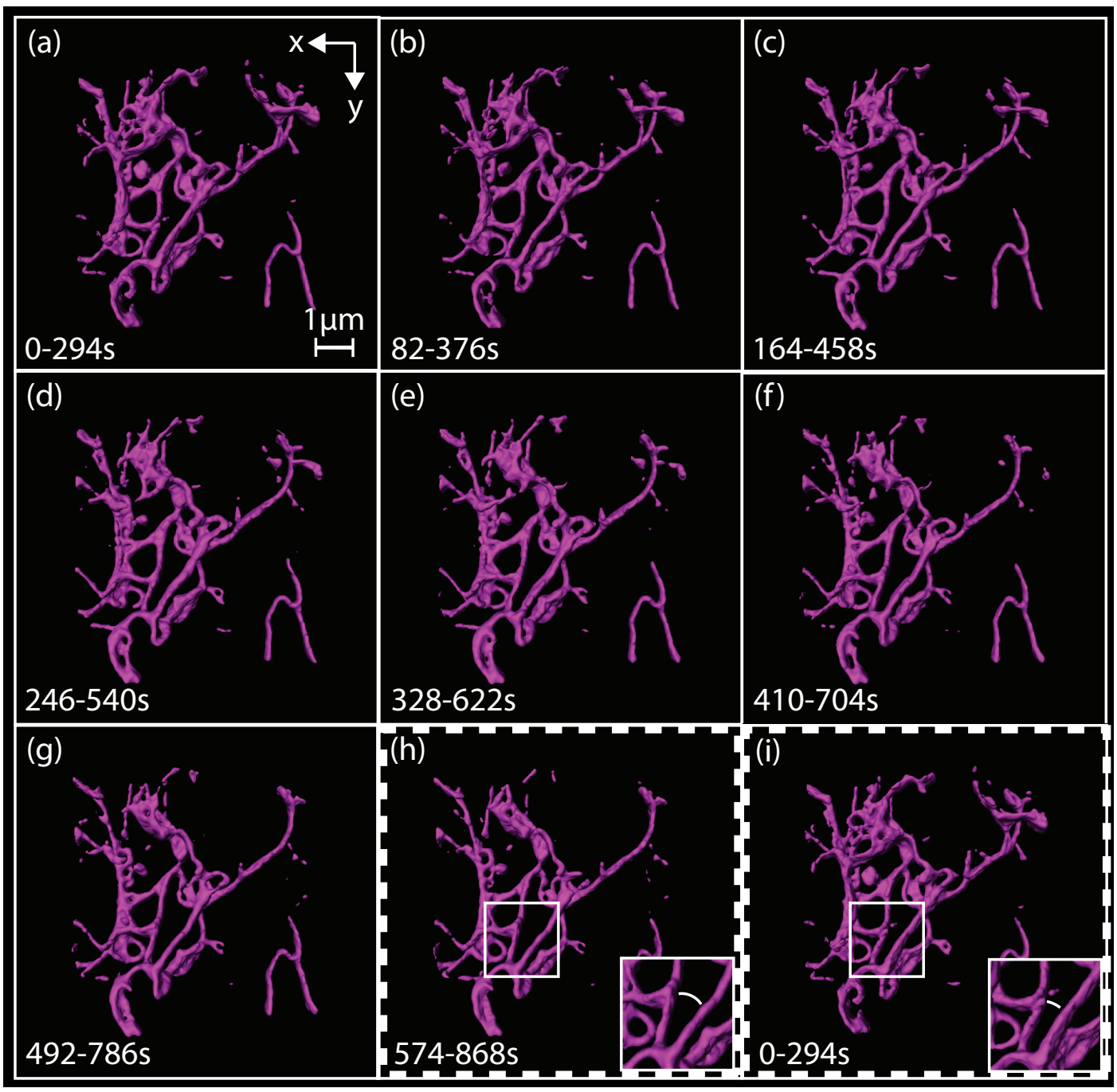

Figure 4.18: Time-series of a 3D surface-rendered reconstruction of the vimentin network in a living HeLa cell. Panels (a) to (h) present a floating average, such that the time interval between the starting points of consecutive panels was $82 \mathrm{~s}$, while the total recording time for each panel was $4.9 \mathrm{~min}$. Note that the first panel (a) is repeated at the bottom right (i), which facilitates assessing the structural change over time. The inset, for example, highlights two bundles of filaments which changed from a v-shaped configuration to running almost parallel to each other. 



\section{Summary and outlook}

Until today, far-field fluorescence microscopy is the key technique to image 3D structures non-invasively and with high molecular specificity. Studies, especially in the life sciences, considerably profit from the current advancements in the field concerning imaging speed and resolution. This thesis extended two different high resolution concepts, namely 4Pi and SMS microscopy, and particularly improved their applicability to the life sciences.

A fast high resolution MMM 4Pi was introduced which combines axial superresolution with an unprecedented frame acquisition up to the video rate $(25 \mathrm{~Hz})$. The MMM 4Pi offers two imaging modes which both feature a 6-fold increase of the axial resolution compared to $2 \mathrm{P}$ microscopy. The first mode sharpens the PSF by the coherent use of two opposing objective lenses for illumination (4Pi type $\mathrm{A}$ ) in conjunction with postprocessing-confocalization. The second mode relies on the coherent use of both objective lenses for the illumination and detection (4Pi type $\mathrm{C}$ ) without additional confocalization.

Both modes had not been shown before and succeeded in reducing the relative side lobe heights to $40-45 \%$ of the main lobe. Therefore, residual ghost images in the recorded data could be unambiguously removed by image post-processing. This was demonstrated by visualizing GFP-tagged mitochondria in live budding yeast cells ( $S$. cerevisiae). The unprecedented acquisition time for a full 3D stack of 200 xy images was $25 \mathrm{~s}$ (including post-processing) for the first and $15.6 \mathrm{~s}$ for the second mode.

The acceleration of the frame rate by a factor of 25-50 compared to former implementations was rendered possible by optimizing both the illumination protocol and the signal detection efficiency. First, utilizing a rotating microlens disk allowed parallelized scanning of the complete field of view within $2.5 \mathrm{~ms}$. Therefore, imaging became only signal-limited. Second, since both imaging modes do not rely on detection pinholes, the overall detection efficiency was significantly enhanced. The 4Pi type $\mathrm{C}$ mode additionally benefits from the doubling of the solid angle used for detection. The SNR was further optimized by the use of a CCD camera with low readout noise and a high quantum efficiency.

The current field of view is only half the size of a preceding implementation. However, this is only limited by the illuminated area and, therefore, by the available laser power and not by the microscope configuration itself. For higher laser powers, the illuminated area can easily be enlarged by increasing the number of foci without reducing the intensity per focus. The laser power can for example be increased by reducing the losses at the optical components in the illumination path or by switching to a more powerful laser.

Each of the two imaging modes has its own peculiarity. The MMM 4Pi type C requires a sophisticated adjustment of the illumination as well as the detection cavities and also de- 
mands a high temporal stability. Particularly, when the experimental schedule is tight, the MMM 4Pi type A with post-processing-confocalization presents a worthwhile alternative since only the adjustment of the illumination cavity is sufficient. However, if quantitative information of the integral intensity or the size and shape of an object, for example, is to be retrieved, the raw data should be retained because the post-processed image is not a direct convolution of the object with the effective PSF of the imaging system. If complete quantitative information is not needed, the 40 -fold increase of the required data volume can be avoided by online pre-processing. In the cases in which the measurement is limited by the SNR, the 4Pi type $\mathrm{C}$ mode is preferable because the detected fluorescence signal is typically doubled. Further, quantitative information can be readily obtained from the stored data.

In future, the imaging speed of MMM 4Pi microscopy will directly benefit from further progress in the laser industry. If more powerful lasers are available, either the illuminated area can be enlarged as mentioned above, or the inter-foci distance can be decreased while maintaining the same field of view. The latter will directly result in shorter acquisition times because the effective pixel dwell time will be increased. Interference between the adjacent foci can be avoided by time-multiplexing. Recent advancements in camera development have achieved effectively zero readout noise and a high quantum efficiency in the visible range. Yet, there is still potential for improving the frame rate, which will accelerate the image acquisition provided that the measurement is not governed by the $\mathrm{SNR}$, but limited by the camera's maximum frame rate.

The second concept for nanoscale far-field fluorescence imaging, which has been dealt with within the framework of this thesis, relies on the switching of single markers in order to spatio-temporally separate neighboring (identical) emitters: SMS microscopy. This work established a novel imaging protocol, extended the method to the third dimension and opened up new fields of applications.

In particular, a fast and asynchronous imaging protocol (PALMIRA) was developed, which overcame the previously reported limitations of the method. These were long image acquisition times of 2-12 hours and sensitivity to (diffuse) background signal. TIRF illumination had therefore been preferred for recording, which limited the method to $2 \mathrm{D}$ specimens.

The PALMIRA imaging protocol reduced the background by matching the camera acquisition time to the average duration of a photon burst. Thereby, dedicated background suppression techniques became obsolete. Consequently, the use of a standard wide-field setup was feasible which rendered imaging inside intact cells possible. By employing the fast reversibly photoswitching fluorescent protein rsFastLime in combination with a non-triggered recording of spontaneous off-on-off cycles without synchronization of the detector, it was possible to accelerate the acquisition time 100-fold to an overall image acquisition time of 2-2.5 min.

This decrease of the measurement time lessened the requirements on the long-term stability of the microscope. The experimental implementation was further simplified because the excitation laser light not only excites but also induces the switching process in 
rsFastLime due to a switch-on crosstalk. Therefore, only one laser line was sufficient for switching on the proteins, reading them out and switching them off.

The concept was extended to 3D imaging by incorporating an estimation for the axial marker position. By employing two offset detection channels, a volume of up to $1200 \mathrm{~nm}$ in depth was imaged at once with high linearity and without any need for axial scanning. The positioning accuracy of up to $14 \mathrm{~nm}$ (FWHM) laterally and $48 \mathrm{~nm}$ (FWHM) axially for a burst of ca. 1600 detected photons is 16-fold and 12-fold, respectively, better than the resolution of a conventional confocal microscope.

By additionally axially scanning the sample in conjunction with two-photon induced on-switching of the markers, an even larger axial depth became accessible for imaging. Although this protocol has the obvious advantage to spatially confine the switching events to the range where axial position determination is possible, it has not been reported yet.

For the first time, 3D SMS imaging was applied to the structural investigation of selforganized 3D colloidal crystals which were constituted by nanoparticles of about $190 \mathrm{~nm}$ diameter. Further, the experimental platform was first used for 3D imaging of live mammalian cells. In particular, the motion of fluorescent protein tagged vimentin-network and keratin6a-network were visualized with an unprecedented resolution. These recordings present the first 3D SMS images of living cells.

In future, SMS microscopy will directly benefit from further development of better markers and cameras. Since the label itself determines the sharpness of the image, the development and use of (reversibly) switchable markers with brighter and shorter photon bursts will directly lead to sharper images and to an acceleration of the image acquisition. Faster detectors will speed up the method even further.

Since this method allows an investigation of single molecule properties in combination with their spatial distribution, the degree of heterogeneity within a population can be assessed. This is usually difficult to determine from bulk measurements. This possibility has already been exploited with respect to the marker's spectral properties and polarization states and will be utilized in future with regard to, for example, the marker's lifetime or its environment sensitive properties.

Because the positioning accuracy scales with the spatial extent of the detection PSF, SMS microscopy will consequently profit from sharpening the PSF, for example, by the coherent use of two opposing lenses. The combination of SMS and 4Pi microscopy has already been reported and will definitely benefit from further developments in the future. 



\section{Bibliography}

[1] E. Abbe. Beiträge zur Theorie des Mikroskops und der mikroskopischen Wahrnehmung. Arch. Mikr. Anat., 9:413-468, 1873. 1, 37

[2] P. Török, P. Varga, Z. Laczik, and G. R. Booker. Electromagnetic diffraction of light focused through a planar interface between materials of mismatched refraction indices: an integral representation. J. Opt. Soc. Am. A, 12(2):325-332, 1995. $1,17,18$

[3] S. W. Hell. Double-scanning confocal microscope. European Patent, 0491289, 1990/1992. US Patent, No 0491289. 1

[4] S. Hell and E. H. K. Stelzer. Fundamental improvement of resolution with a 4Piconfocal fluorescence microscope using two-photon excitation. Opt. Commun., 93:277-282, 1992. 1

[5] M.G.L. Gustafsson, D.A. Agard, and J.W. Sedat. I5M: 3D widefield light microscopy with better than $100 \mathrm{~nm}$ axial resolution. J. Microsc., 195:10-16, 1999. 1,17

[6] L. Shao, B. Isaac, S. Uzawa, D. A. Agard, J. W. Sedat, and M. G. L. Gustafsson. I5S: Wide-field light microscopy with 100-nm-scale resolution in three dimensions. Biophysical Journal, 94(12):4971-4983, 2008. 2

[7] S. W. Hell. Increasing the resolution of far-field fluorescence light microscopy by point-spread-function engineering. In J.R. Lakowicz, editor, Topics in Fluorescence Spectroscopy, volume 5, pages 361-422. Plenum Press, New York, 1997. 2

[8] S. W. Hell. Improvement of lateral resolution in far-field light microscopy using two-photon excitation with offset beams. Opt. Commun., 106:19-24, 1994. 2

[9] S.W. Hell. Strategy for far-field optical imaging and writing without diffraction limit. Phys. Lett. A, 326(1-2):140-145, 2004. 2

[10] Stefan W. Hell. Far-field optical nanoscopy. Science, 316(5828):1153-1158, 2007. $2,38,44$

[11] S. W. Hell and J. Wichmann. Breaking the diffraction resolution limit by stimulated emission: stimulated emission depletion fluorescence microscopy. Opt. Lett., 19(11):780-782, 1994. 2, 37 
[12] Rainer Heintzmann, Thomas M. Jovin, and Christoph Cremer. Saturated patterned excitation microscopy - a concept for optical resolution improvement. J. Opt. Soc. Am. A: Optics and Image Science, and Vision, 19(8):1599-1609, 2002. TY - JOUR. 2

[13] M. G. L. Gustafsson. Nonlinear structured-illumination microscopy: Wide-field fluorescence imaging with theoretically unlimited resolution. Proc. Natl. Acad. Sci. USA, 102(37):13081-13086, 2005. 2

[14] L. Schermelleh, P. M. Carlton, S. Haase, L. Shao, L. Winoto, P. Kner, B. Burke, M. C. Cardoso, D. A. Agard, M. G. L. Gustafsson, H. Leonhardt, and J. W. Sedat. Subdiffraction multicolor imaging of the nuclear periphery with $3 \mathrm{~d}$ structured illumination microscopy. Science, 320(5881):1332-1336, 2008. 2

[15] S.W. Hell, S. Jakobs, and L. Kastrup. Imaging and writing at the nanoscale with focused visible light through saturable optical transitions. Appl. Phys. A, 77:859$860,2003.2,38$

[16] S. W. Hell and M. Kroug. Ground-state depletion fluorescence microscopy, a concept for breaking the diffraction resolution limit. Appl. Phys. B, 60:495-497, 1995. 2,37

[17] S. Bretschneider, C. Eggeling, and S. W. Hell. Breaking the diffraction barrier in fluorescence microscopy by optical shelving. Phys. Rev. Lett., 98:218103, 2007. 2, 37

[18] M. Hofmann, C. Eggeling, S. Jakobs, and S.W. Hell. Breaking the diffraction barrier in fluorescence microscopy at low light intensities by using reversibly photoswitchable proteins. Proc. Natl. Acad. Sci. USA, 102(49):17565-17569, 2005. 2, 37

[19] E. Betzig, G.H. Patterson, R. Sougrat, O.W. Lindwasser, S. Olenych, J.S. Bonifacino, M.W. Davidson, J. Lippincott-Schwartz, and H.F. Hess. Imaging intracellular fluorescent proteins at nanometer resolution. Science, 313(5793):1642-1645, 2006. $2,40,44,45,46$

[20] Samuel T. Hess, Thanu P. K. Girirajan, and Michael D. Mason. Ultra-high resolution imaging by fluorescence photoactivation localization microscopy. Biophys. J., 91(11):4258-4272, 2006. 2, 40, 41, 45

[21] Michael J. Rust, Mark Bates, and Xiaowei Zhuang. Sub-diffraction-limit imaging by stochastic optical reconstruction microscopy (STORM). Nat. Methods, 3:793796, 2006. 2, 40, 44, 45

[22] M. Lang, T. Müller, J. Engelhardt, and S. W. Hell. 4Pi microscopy of type A with 1photon excitation in biological fluorescence imaging. Optics Express, 15(5):24592467, 2007. 3, 17 
[23] M. Lang, J. Engelhardt, and S. W. Hell. 4Pi microscopy with linear fluorescence excitation. Opt. Lett., 32(3):259-261, 2007. 3, 17

[24] M. C. Lang. 4Pi-Mikroskopie mit ultra-hohen Aperturwinkeln: Elimination der Nebenmaxima und Effizienzsteigerung. PhD thesis, Ruprecht-Karls-Universität Heidelberg, 2007. 3

[25] A. Egner, S. Jakobs, and S.W. Hell. Fast 100-nm resolution 3D-microscope reveals structural plasticity of mitochondria in live yeast. Proc. Natl. Acad. Sci. USA, 99:3370-3375, 2002. 3, 17, 30

[26] Peter Atkins and Julio de Paula. Atkins' Physical Chemistry. Oxford University Press, 7th edition, 2002. 5

[27] B. Valeur. Molecular fluorescence. Wiley-VCH Verlag, Weinheim, 1st edition, 2002. 5

[28] A. Diaspro, G. Chirico, C. Usai, P. Ramoino, and J. Dobrucki. Photobleaching. In James. B. Pawley, editor, Handbook of biological confocal microscopy, pages 690-702. Springer, 3rd edition, 2006. 7

[29] Max Born and Emil Wolf. Principles of Optics. Cambridge University Press, Cambridge, New York, Melbourne, Madrid, Cape Town, 7th edition, 2002. 7

[30] J. W. Goodman. Introduction to Fourier optics. Mc Graw Hill, 1968. 7, 8

[31] M. Gu. Principles of three-dimensional imaging in confocal microscopes. World Scientific, Singapore, 1996. 7

[32] S.G. Lipson, H. Lipson, and D.S. Tannhauser. Optical Physics. Cambridge University Press, 3rd edition, 1995. 7, 9

[33] T. Wilson (ed.). Confocal microscopy. Academic Press Ltd., London, 1990. 7

[34] Alexander Egner. Multifokale hochauflösende 3D-Fluoreszenzmikroskopie. Dissertation, Ruprecht-Karls-Universität, 2002. 7, 22

[35] M. Minsky. Microscopy apparatus, US Patent 3,013,467, 1961. 12

[36] S.W. Hell. Hochauflösende 3D-Lichtmikroskopie. In J. Bille and Schlegl W., editors, Medizinische Physik 3: Medizinische Laserphysik, pages 179-215. Springer, Berlin, 2009. 12

[37] C.E. Shannon. Communication in the presence of noise. Proc. Inst. Radio. Eng., 37(1):10 - 21, 1949. 13, 29, 43

[38] M. Göppert-Mayer. Über Elementarakte mit zwei Quantensprüngen. Ann. Phys. (Leipzig), 9:273-295, 1931. 14, 66 
[39] W. Kaiser and C. B. Garret. Two-photon excitation in CaF2:Eu2+. Phys. Rev. Lett., 7:229-231, 1961. 14

[40] W. Denk, J. H. Strickler, and W. W. Webb. Two-photon laser scanning fluorescence microscopy. Science, 248:73-76, 1990. 15, 66

[41] B. Bailey, D. L. Farkas, D. L. Taylor, and F. Lanni. Enhancement of axial resolution in fluorescence microscopy by standing-wave excitation. Nature, 366:44-48, 1993. 17

[42] S. W. Hell, S. Lindek, and E. H. K. Stelzer. Enhancing the axial resolution in far-field light microscopy: two-photon excitation 4Pi-confocal fluorescence microscopy. J. Mod Opt., 41(4):675-681, 1994. 17

[43] A. Egner, S. Verrier, A. Goroshkov, H.-D. Söling, and S.W. Hell. 4Pi microscopy of the Golgi apparatus in live mammalian cells. J. Struct. Biol., 147(1):70-76, 2004. 18

[44] G. Perinetti, T. Muller, A. Spaar, R. Polishchuk, A. Luini, and A. Egner. Correlation of 4Pi and Electron Microscopy to Study Transport Through Single Golgi Stacks in Living Cells with Super Resolution. Traffic, 10(4):379-391, 2009. 18

[45] E. Wolf. Electromagnetic diffraction in optical systems i. an integral representation of the image field. Proc. R. Soc. Lond. A, 253:349-357, 1959. 20

[46] B. Richards and E. Wolf. Electromagnetic diffraction in optical systems II. Structure of the image field in an aplanatic system. Proc. R. Soc. Lond. A, 253:358-379, 1959. 20

[47] M. Nagorni and S.W. Hell. Coherent use of opposing lenses for axial resolution increase in fluorescence microscopy. ii. power and limitation of nonlinear image restoration. J. Opt. Soc. Am. A, 18(1):49-54, 2001. 22

[48] P. E. Hänninen, S. W. Hell, J. Salo, E. Soini, and C. Cremer. Two-photon excitation 4Pi-confocal microscope: Enhanced axial resolution microscope for biological research. Appl. Phys. Lett., 66:1698-1700, 1995. 22

[49] W. H. Richardson. Bayesian-based iterative method of image restoration. J. Opt. Soc. Am., 62:55-59, 1972. 22

[50] A. Egner, V. Andresen, and S.W. Hell. Comparison of the axial resolution of practical nipkow-disk confocal fluorescence microscopy with that of multifocal multiphoton microscopy: theory and experiment. J. Microsc., 206:24-32, 2002. 23

[51] K. König, T. W. Becker, P. Fischer, I. Riemann, and K-J Halbhuber. Pulse-length dependence of cellular response to intense near-infrared laser pulses in multiphoton microscopes. Opt. Lett., 24(2):113-115, 1999. 24, 30 
[52] E. Bonet, P. Andrés, J.C. Barreiro, and A. Pons. Self-imaging properties of a periodic microlens array: versatile array illuminator realization. Opt. Commun., 106:39-44, 1994. 24

[53] M.A.A. Neil, R. Juskaitis, and T. Wilson. Method of obtaining optical sectioning by using structured light in a conventional microscope. Opt. Lett., 22:1905-1907, 1997. 24

[54] R. Heintzmann. Structured illumination methods. In James. B. Pawley, editor, Handbook of biological confocal microscopy, pages 265-279. Springer, 3rd edition, 2006. 24

[55] R. Heintzmann and P. A. Benedetti. High-resolution image reconstruction in fluorescence microscopy with patterned excitation. Applied Optics, 45(20):5037-5045, 2006. 24

[56] A. Egner, M. Schrader, and S. W. Hell. Refractive index mismatch induced intensity and phase variations in fluorescence confocal, multiphoton and $4 \mathrm{Pi}-\mathrm{mi}$ croscopy. Opt. Commun., 153:211-217, 1998. 29

[57] S. Jakobs, N. Martini, A.C. Schauss, A. Egner, B. Westermann, and S.W. Hell. Spatial and temporal dynamics of budding yeast mitochondria lacking the division component fis 1p. J. Cell Sci., 116:2005-2014, 2003. 30

[58] H. Kawano, Y. Nabekawa, A. Suda, Y. Oishi, H. Mizuno, A. Miyawaki, and K. Midorikawa. Attenuation of photobleaching in two-photon excitation fluorescence from green fluorescent protein with shaped excitation pulses. Biochem. Biophys. Res. Commun., 311:592-596, 2003. 30

[59] Nicole Ehrmann. Neue Methoden der multifokalen Mikroskopie. Master's thesis, Universität Kassel, 2007. 35

[60] S.W. Hell. Toward fluorescence nanoscopy. Nature Biotechnol., 21(11):13471355, 2003. 37

[61] W. Heisenberg. Prinzipien der Quantentheorie. Hirzel-Verlag, Leipzig, Germany, 1930. 40

[62] N. Bobroff. Position measurement with a resolution and noise-limited instrument. Rev. Sci. Instrum., 57(6):1152-1157, 1986. 40

[63] E. Betzig. Proposed method for molecular optical imaging. Optics Letters, 20(3):237-239, 1995. 40

[64] S. W. Hell, J. Soukka, and P. E. Hänninen. Two- and multiphoton detection as an imaging mode and means of increasing the resolution in far-field light microscopy. Bioimaging, 3:65-69, 1995. 40 
[65] T. Schmidt, G. J. Schutz, W. Baumgartner, H. J. Gruber, and H. Schindler. Imaging of single molecule diffusion. Proc. Natl. Acad. Sci. USA, 93(7):2926-9, 1996. 40

[66] M. S. Robbins and B. J. Hadwen. The noise performance of electron multiplying charge-coupled devices. IEEE Trans. Electron. Dev., 50(5):1227-1232, 2003. 43

[67] R.E. Thompson, D.R. Larson, and W.W. Webb. Precise nanometer localization analysis for individual fluorescent probes. Biophys. J., 82:2775-2783, 2002. 43, 49,50

[68] C. v. Middendorff. Experimental Stochastics in High Resolution Fluorescence Microscopy. PhD thesis, Ruprecht-Karls-Universität Heidelberg, 2008. 43, 44, 62, 65

[69] M. F. Juette, T. J. Gould, M. D. Lessard, M. J. Mlodzianoski, B. S. Nagpure, B. T. Bennett, S. T. Hess, and J. Bewersdorf. Three-dimensional sub-100 nm resolution fluorescence microscopy of thick samples. Nature Methods, 5(6):527-529, 2008. 44,55

[70] W. E. Moerner. Single-molecule mountains yield nanoscale cell images. Nat Methods, 3(10):781-2, 2006. 1548-7091 (Print) Comment News. 44

[71] H. Shroff, C. G. Galbraith, J. A. Galbraith, H. White, J. Gillette, S. Olenych, M. W. Davidson, and E. Betzig. Dual-color superresolution imaging of genetically expressed probes within individual adhesion complexes. Proc. Natl. Acad. Sci. USA, 104(51):20308-20313, 2007. 44

[72] M. Bates, B. Huang, G.P. Dempsey, and X. Zhuang. Multicolor super-resolution imaging with photo-switchable fluorescent probes. Science, 317:1749-1753, 2007. 44

[73] A. C. Stiel, S. Trowitzsch, G. Weber, M. Andresen, C. Eggeling, S. W. Hell, S. Jakobs, and M. C. Wahl. 1.8 a bright-state structure of the reversibly switchable fluorescent protein dronpa guides the generation of fast switching variants. Biochem. J., 402(1):35-42, 2007. 44, 45, 46, 50, 91

[74] Ryoko Ando, Hideaki Mizuno, and Atsushi Miyawaki. Regulated fast nucleocytoplasmic shuttling observed by reversible protein highlighting. Science, 306(5700):1370-1373, 2004. 45

[75] C. Geisler, A. Schönle, C. von Middendorff, H. Bock, C. Eggeling, A. Egner, and S. W. Hell. Resolution of 1/10 in fluorescence microscopy using fast single molecule photo-switching. Appl. Phys. A, 88(2):223-226, 2007. 46 
[76] A. Egner, C. Geisler, C von Middendorff, H. Bock, D. Wenzel, R. Medda, M. Andresen, A. C. Stiel, S. Jakobs, C. Eggeling, A. Schönle, and S. W. Hell. Fluorescence nanoscopy in whole cells by asnychronous localization of photoswitching emitters. Biophys. J., 93:3285-3290, 2007. 46, 49

[77] I. Testa, A. Schönle, C. V. Middendorff, C. Geisler, R. Medda, C. A. Wurm, A. C. Stiel, S. Jakobs, M. Bossi, C. Eggeling, S. W. Hell, and A. Egner. Nanoscale separation of molecular species based on their rotational mobility. Optics Express, 16(25):21093-21104, 2008. 47, 93

[78] H. Bock, C. Geisler, C. A. Wurm, S. Jakobs, A. Schönle, A. Egner, S. W. Hell, and C. Eggeling. Two-color far-field fluorescence nanoscopy based on photoswitching emitters. Appl. Phys. B, 88(161-165), 2007. 47

[79] J.A. Hogbom. Aperture synthesis with a non-regular distribution of interferometer baselines. Astron. Astrophys. Suppl., 15:417-426, 1974. 49

[80] J. Fölling, V. Belov, R. Kunetsky, R. Medda, A. Schönle, A. Egner, C. Eggeling, M. Bossi, and S. W. Hell. Photochromic rhodamines provide nanoscopy with optical sectioning. Angew. Chem. Int. Ed., 46:6266-6270, 2007. 54, 64, 66

[81] J. Fölling, V. Belov, D. Riedel, A. Schönle, A. Egner, C. Eggeling, M. Bossi, and S. W. Hell. Fluorescence nanoscopy with optical sectioning by two-photon induced molecular switching using continuous-wave lasers. ChemPhysChem, 9:321 - 326, 2008. 54

[82] A. Vaziri, J. Y. Tang, H. Shroff, and C. V. Shank. Multilayer three-dimensional super resolution imaging of thick biological samples. Proc. Natl. Acad. Sci. USA, 105(51):20221-20226, 2008. 54, 71

[83] B. Huang, W. Wang, M. Bates, and X. Zhuang. Three-dimensional super-resolution imaging by stochastic optical reconstruction microscopy. Science, 319:810-813, 2008. 54,55

[84] Claas v. Middendorff, Alexander Egner, Claudia Geisler, Stefan W. Hell, and Andreas Schönle. Isotropic 3D nanoscopy based on single emitter switching. Opt. Express, 16(25):20774-20788, 2008. 55

[85] G. Shtengel, J. A. Galbraith, C. G. Galbraith, J. Lippincott-Schwartz, J. M. Gillette, S. Manley, R. Sougrat, C. M. Waterman, P. Kanchanawong, M. W. Davidson, R. D. Fetter, and H. F. Hess. Interferometric fluorescent super-resolution microscopy resolves 3D cellular ultrastructure. Proc. Natl. Acad. Sci. USA, 106(9):3125-3130, 2009. 55

[86] S. R. P. Pavani, M. A. Thompson, J. S. Biteen, S. J. Lord, N. Liu, R. J. Twieg, R. Piestun, and W. E. Moerner. Three-dimensional, single-molecule fluorescence 
imaging beyond the diffraction limit by using a double-helix point spread function. Proc. Natl. Acad. Sci. USA, 106(9):2995-2999, 2009. 55

[87] J. Enderlein, E. Toprak, and P. R. Selvin. Polarization effect on position accuracy of fluorophore localization. Optics Express, vol.14, no.18, 2006. 55

[88] B. Huang, S. A. Jones, B. Brandenburg, and X. W. Zhuang. Whole-cell 3D STORM reveals interactions between cellular structures with nanometer-scale resolution. Nature Methods, 5(12):1047-1052, 2008. 55

[89] M. J. Mlodzianoski, M. F. Juette, G. L. Beane, and J. Bewersdorf. Experimental characterization of 3D localization techniques for particle-tracking and superresolution microscopy. Optics Express, 17(10):8264-8277, 2009. 55, 64

[90] J. Chao, S. Ram, A. V. Abraham, E. S. Ward, and R. J. Ober. A resolution measure for three-dimensional microscopy. Optics Communications, 282(9):1751-1761, 2009. 64

[91] V. N. Belov, M. L. Bossi, J. Foelling, V. P. Boyarskiy, and S. W. Hell. Rhodamine spiroamides for multicolor single-molecule switching fluorescent nanoscopy. Chem.Eur.J., 15(15):10762-10776, 2009. 65

[92] S. C. Glotzer and M. J. Solomon. Anisotropy of building blocks and their assembly into complex structures. Nature Materials, 6(8):557-562, 2007. 69

[93] A. Van blaaderen and P. Wiltzius. Real-space structure of colloidal hard-sphere glasses. Science, 270(5239):1177-1179, 1995. 69

[94] Y. L. Wu, D. Derks, A. van Blaaderen, and A. Imhof. Melting and crystallization of colloidal hard-sphere suspensions under shear. Proc. Natl. Acad. Sci. USA, 106(26):10564-10569, 2009. 69

[95] A. van Blaaderen. Materials science - colloids get complex. Nature, 439(7076):545-546, 2006. 69

[96] V. Prasad, D. Semwogerere, and E. R. Weeks. Confocal microscopy of colloids. Journal of Physics-Condensed Matter, 19(11), 2007. 69

[97] A. M. Alsayed, M. F. Islam, J. Zhang, P. J. Collings, and A. G. Yodh. Premelting at defects within bulk colloidal crystals. Science, 309(5738):1207-1210, 2005. 69

[98] B. Harke, J. Ullal, J. Keller, and S. W. Hell. Three-dimensional nanoscopy of colloidal crystals. Nano Lett., DOI: 10.1021/n1073164n, 2008. 69

[99] J. Fölling, S. Polyakova, V. Belov, A. van Blaaderen, M. Bossi, and S. W. Hell. Synthesis and characterization of photoswitchable fluorescent silica nanoparticles. Small, 4(1):134-142, 2008. 69 
[100] M. Bossi, J. Folling, V. N. Belov, V. P. Boyarskiy, R. Medda, A. Egner, C. Eggeling, A. Schönle, and S. W. Hell. Multicolor far-field fluorescence nanoscopy through isolated detection of distinct molecular species. Nano Letters, 8(8):2463-2468, 2008. 69

[101] H. Shroff, C. G. Galbraith, J. A. Galbraith, and E. Betzig. Live-cell photoactivated localization microscopy of nanoscale adhesion dynamics. Nature Methods, 5(5):417-423, 2008. 71

[102] B. Hein, K. I. Willig, and S. W. Hell. Stimulated emission depletion (sted) nanoscopy of a fluorescent protein-labeled organelle inside a living cell. Proc. Natl. Acad. Sci. USA, 105(38):14271-14276, 2008. 73

[103] B. Alberts, A. Johnson, J. Lewis, M. Raff, K. Roberts, and P. Walter. Molecular Biology of the Cell. Garland Science, New York, 4 edition, 2002. 73

[104] M. L. Styers, A. P. Kowalczyk, and V. Faundez. Architecture of the vimentin cytoskeleton is modified by perturbation of the gtpase arf1. Journal of Cell Science, 119(17):3643-3654, 2006. 73

[105] Y. H. Chou, F. W. Flitney, L. Chang, M. Mendez, B. Grin, and R. D. Goldman. The motility and dynamic properties of intermediate filaments and their constituent proteins. Experimental Cell Research, 313(10):2236-2243, 2007. 73

[106] M. Yoon, R. D. Moir, V. Prahlad, and R. D. Goldman. Motile properties of vimentin intermediate filament networks in living cells. Journal of Cell Biology, 143(1):147$157,1998.73$

[107] K. H. Yoon, M. Yoon, R. D. Moir, S. Khuon, F. W. Flitney, and R. D. Goldman. Insights into the dynamic properties of keratin intermediate filaments in living epithelial cells. Journal of Cell Biology, 153(3):503-516, 2001. 73

[108] J. Wiedenmann, S. Ivanchenko, F. Oswald, F. Schmitt, C. Röcker, A. Salih, K.-D. Spindler, and G.U. Nienhaus. Eosfp, a fluorescent marker protein with uv-inducible green-to-red fluorescence conversion. Proc. Natl. Acad. Sci. USA, 101(45):1590515910, 2004. 73

[109] N. G. Gurskaya, V. V. Verkhusha, A. S. Shcheglov, D. B. Staroverov, T. V. Chepurnykh, A. F. Fradkov, S. Lukyanov, and K. A. Lukyanov. Engineering of a monomeric green-to-red photoactivatable fluorescent protein induced by blue light. Nature Biotechnology, 24(4):461-465, 2006. 73

[110] K. Nienhaus, G. U. Nienhaus, J. Wiedenmann, and H. Nar. Structural basis for photo-induced protein cleavage and green-to-red conversion of fluorescent protein eosfp. Proc. Natl. Acad. Sci. USA, 102(26):9156-9159, 2005. 73 
[111] V. Adam, K. Nienhaus, D. Bourgeois, and G. U. Nienhaus. Structural basis of enhanced photoconversion yield in green fluorescent protein-like protein dendra2. Biochemistry, 48(22):4905-4915, 2009. 73

[112] B. Westermann and W. Neupert. Mitochondria-targeted green fluorescent proteins: convenient tools for the study of organelle biogenesis in saccharomyces cerevisiae. Yeast, 16(15):1421-1427, 2000. Using Smart Source Parsing 2000 Nov. 91

[113] D. E. Drew, G. von Heijne, P. Nordlund, and J. W. de Gier. Green fluorescent protein as an indicator to monitor membrane protein overexpression in escherichia coli. FEBS Lett, 507(2):220-4, 2001. 0014-5793 (Print) Journal Article Research Support, Non-U.S. Gov't. 91

[114] K. T. Tokuyasu. A technique for ultracryotomy of cell suspensions and tissues. $J$ Cell Biol, 57(2):551-65, 1973. 0021-9525 (Print) Journal Article. 91

[115] K. T. Tokuyasu. Immunochemistry on ultrathin frozen sections. Histochem J, 12(4):381-403, 1980. 91

[116] W. Liou, H. J. Geuze, and J. W. Slot. Improving structural integrity of cryosections for immunogold labeling. Histochem Cell Biol, 106(1):41-58, 1996. 0948-6143 (Print) Journal Article Research Support, Non-U.S. Gov't Review. 92

[117] M. Osborn, W. W. Franke, and K. Weber. Visualization of a system of filaments 7-10 nm thick in cultured cells of an epithelioid line (ptk2) by immunofluorescence microscopy. Proc. Natl. Acad. Sci. USA, 74(6):2490-4, 1977. 0027-8424 (Print) Journal Article. 92

[118] K. Weber, T. Bibring, and M. Osborn. Specific visualization of tubulin-containing structures in tissue culture cells by immunofluorescence. cytoplasmic microtubules, vinblastine-induced paracrystals, and mitotic figures. Exp Cell Res, 95(1):111-20, 1975. 0014-4827 (Print) Journal Article Research Support, U.S. Gov't, P.H.S. 92, 93

[119] Joseph Sambrook and David W. Russel. Molecular Cloning: A Laboratory Manual. Cold Spring Harbor Laboratory, 3rd edition, 2000. 92 


\section{A Protocols for sample preparation}

\section{Cultivation of Saccharomyces cerevisiae}

Unsynchronized cultures of diploid cells (yeast strain BY4743 (MATa/MAT $\alpha$; his $3 \delta 1 /$ his3 $\delta 1$; leu2 $\delta 0 /$ leu2 $\delta 0$; met15 $\delta 0 /$ MET15; LYS2/lys2 $\delta 0$; ura3 $\delta 0 /$ ura3 $\delta 0)$ ) were grown to the midlogarithmic phase in batch cultures in SC-URA (70\% glucose, $17 \%\left(\mathrm{NH}_{4}\right) 2 \mathrm{SO}_{4}$, $6 \%$ yeast nitrogen base, $7 \%$ drop-out-mixture without uracil) full medium. The cells had been transformed with the plasmid pVT100-mtGFP for constitutive expression of mitochondria-targeted GFP [112].

\section{rs-FastLime protein production and purification}

The RSFP rsFastLime was expressed in the Escherichia coli strain HMS 174 (DE3) and purified by Ni-NTA affinity chromatography and subsequent size-exclusion chromatography according to standard procedures [73]. The purified proteins were concentrated to $33 \mathrm{mg} / \mathrm{ml}$ and taken up in $100 \mathrm{mM}$ Tris- $\mathrm{HCl}, 150 \mathrm{mM} \mathrm{NaCl}, \mathrm{pH}$ 7.5.

\section{Preparation of cryosections of cytoplasmic membrane labeled $E$. coli}

In order to label the cytoplasmic membrane with the RSFP rsFastLime, a fusion protein consisting of the M13 bacteriophage procoat protein fused to rsFastLime was expressed. M13 is integrated into the cytoplasmic membrane. It has two membrane-spanning domains with both termini reaching into the cytoplasm. Therefore, the coding sequence for rsFastLime [73] was PCR amplified and inserted into a modified pET28 expression vector harboring a M13-GFP fusion [113] to replace the GFP coding sequence. The fusion protein was expressed in SURE E. coli cells (Stratagene, La Jolla, CA, USA). Thin cryosections were prepared as described previously [114][115]. E. coli cells were fixed with $2 \%(\mathrm{w} / \mathrm{v})$ PFA (1 vol growth medium plus $1 \mathrm{vol} 4 \%(\mathrm{w} / \mathrm{v})$ PFA) for $30 \mathrm{~min}$ at room temperature. After centrifugation, cells were postfixed with $4 \%(\mathrm{w} / \mathrm{v})$ and $0.1 \%(\mathrm{w} / \mathrm{v})$ glutaraldehyde in PBS for $2 \mathrm{~h}$ on ice. After being washed twice with PBS- $0.02 \%$ glycine, cells were embedded in $10 \%(\mathrm{w} / \mathrm{v})$ gelatin, cooled on ice and cut into small blocks. The blocks were infused with $2.3 \mathrm{M}$ sucrose in PBS at $4{ }^{\circ} \mathrm{C}$ overnight, mounted on metal pins and frozen in liquid nitrogen. $200 \mathrm{~nm}$ sections were cut at $-110^{\circ} \mathrm{C}$ using a diamond 
knife (Diatome, Biel, Switzerland) in an ultracryomicrotome (Leica Mikrosysteme, Wetzlar, Germany) and collected using a 1:1 mixture of $2.3 \mathrm{M}$ sucrose [116] and $2 \%(\mathrm{w} / \mathrm{v})$ methyl cellulose containing $40 \mathrm{mM}$ Cysteamine (BioChemika, $\geq 98.0 \%$ (RT) (SigmaAldrich)). The cryosections were deposited on electric glow discharge (minus) treated cover slips, which were rinsed beforehand with deionized water for $5 \mathrm{~min}$ and cleaned in a low pressure plasma system (Femto-RF, Diener Electronic, Nagold, Germany). The mixture of methyl cellulose and sucrose which completely covered the sample was removed mechanically.

\section{Labeling of $\alpha$-tubulin with rsFastLime}

PtK2 cells were grown as described previously [117]. Cells were seeded on coverslips (Menzel, Braunschweig, Germany) in a six-well plate (Nunc, Wiesbaden, Germany) and grown to a confluence of ca. $80 \%$. For immunofluorescence labeling, cells were fixated for 4-6 min in icecold methanol (abs) and subsequently blocked for $10 \mathrm{~min}$ in PBS containing $1 \%(\mathrm{w} / \mathrm{v})$ BSA (blocking buffer). Cells were incubated with primary antibodies ( $2 \mathrm{mg} / \mathrm{ml}$, anti- $\alpha$-tubulin mouse IgG biotin conjugated, Invitrogen, Carlsbad, CA, USA) diluted in blocking buffer at room temperature for $1 \mathrm{~h}$ followed by PBS washes and 5 min blocking in blocking buffer. For bridging the biotinylated anti- $\alpha$-tubulin antibody with biotinylated rsFastLime, cells were incubated with Neutravidin $(10 \mathrm{mg} / \mathrm{ml}$, Invitrogen) at room temperature for $1 \mathrm{~h}$ followed by a short fixation with $3.7 \%$ (w/v) PFA at room temperature for $5 \mathrm{~min}$ and subsequent PBS washes. Finally, cells were incubated with biotinylated rsFastLime $(50 \mathrm{mg} / \mathrm{ml}$, FluoReporter Mini-biotin-XX Protein Labeling Kit, Invitrogen) at room temperature for $1 \mathrm{~h}$.

\section{Immunostaining of $\beta$-tubulin with SRA577}

The mammalian PtK2 cell line was grown as described previously [118]. Cells were seeded on standard glass cover slips to a confluence of about $80 \%$ and fixed with icecold methanol (abs) for 4 min followed by an incubation in blocking buffer (PBS containing $1 \%(\mathrm{w} / \mathrm{v}) \mathrm{BSA})$. Immunostaining of microtubules was performed with anti- $\beta$-tubulin mouse IgG (Sigma-Aldrich Chemie Gmbh, München, Germany) as primary antibody and with SRA577-conjugated sheep anti-mouse IgG as secondary antibody. Both antibodies were diluted in blocking buffer and incubated for $1 \mathrm{~h}$ each followed by several washes in blocking buffer.

\section{DNA-constructs}

Vimentin-Dendra2 and Keratin6a-tdEosFP:

Standard methods were used for cloning [119]. In order to tag keratin6a and vimentin at the C-terminus, the expression plasmids pMD-keratin6a-Dendra2 and pMD-Vimentin- 
tdEosFP, were constructed by Gateway vector conversion (Invitrogen, Carlsbad, CA, USA) from the donor vector pDONR223-keratin6a, pDONR223-VIM and the empty destination vector $\mathrm{pMD}$-GATEWAY-Dendra 2 and $\mathrm{pMD}$-GATEWAY-EosFP. The vector pMDGATEWAY-Dendra2-N was created previously by exchange of the coding sequence of tdEosFP to Dendra2 within the vector pMD-GATEWAY-tdEosFP-N [77].

\section{Cell culture and transfection}

The mammalian PtK2 cell line was grown as previously described [118]. For transfection, PtK2 cells were grown overnight on glass cover slips. After reaching ca. $80 \%$ confluence, the plasmids were introduced using the Nanofectin kit according to the manufacturer's instructions (PAA, Pasching, Austria). The cells were incubated for at least $24 \mathrm{~h}$ before imaging. 



\section{List of publications}

Parts of this thesis have been published as follows:

- Geisler, C., A. Schönle, C. von Middendorf, H. Bock, C. Eggeling, A. Egner, S.W. Hell (2007): "Resolution of $\lambda / 10$ in fluorescence microscopy using fast single molecule photo-switching". Appl. Phys. A 88, 223-226.

- Egner, A., C. Geisler, C. von Middendorff, H. Bock, D. Wenzel, R. Medda, M. Andresen, A. C. Stiel, S. Jakobs, C. Eggeling, A. Schönle, S. W. Hell (2007): "Fluorescence nanoscopy in whole cells by asynchronous localization of photoswitching emitters". Biophys. J. 93, 3285 - 3290.

- Bock, H., C. Geisler, C. Wurm, S. Jakobs, A. Schönle, A. Egner, S.W. Hell, C. Eggeling (2007): "Two-color far-field fluorescence nanoscopy based on photoswitching emitters". Appl. Phys. B 88, 161-165.

- Middendorff, C. v, A. Egner, C. Geisler, S. W. Hell, A. Schönle (2008): "Isotropic 3D nanoscopy based on single emitter switching". Opt. Expr. 16 (25), 2077420788.

- Testa, I., A. Schönle, C. v. Middendorff, C. Geisler, R. Medda, C. A. Wurm, A. C. Stiel, S. Jakobs, M. Bossi, C. Eggeling, S. W. Hell, A. Egner (2008): "Nanoscale separation of molecular species based on their rotational mobility". Opt. Expr. 16 (25), 21093 - 21104.

Talks on conferences:

- Geisler, C., C. v. Middendorff, A. Schönle, A. Egner, S.W. Hell , "Optical sectioning in 3D far-field fluorescence nanoscopy based on single marker switching", Focus on Microscopy 2009, Krakow, Poland 



\section{Acknowledgements}

This thesis was performed in the Department of NanoBiophotonics at the Max Planck Institute for Biophysical Chemistry in Göttingen. Many people from different fields have contributed to the success of this thesis and I would like to express my sincere thanks to

... Prof. Stefan W. Hell for giving me the opportunity to work on these projects. He not only provided an outstanding working atmosphere in an excellently equipped laboratory, but also supported with many ideas and encouraged with great enthusiasm.

... Prof. Rainer G. Ulbrich for his lively interest in the projects and for the willingness to be one of the referees of this thesis.

... Alexander Egner for his excellent mentoring during my thesis. He not only set up the initial layout of the experimental implementations in the 4Pi as well as in the SMS project, but also provided a lot of ideas and was a great source of help in every aspect. I further acknowledge his contribution to the SMS analysis software.

... Andreas Schönle for his many ideas, his contributions to the SMS-analysis software and for providing the data acquisition software ImSpector and Claas v. Middendorff and Jan Keller for their contributions to the SMS analysis software.

... Jonas Fölling, Hannes Bock, Ilaria Testa and Daniel Aquino for helpful discussions on various aspects of the 'blinking markers' and Christian Eggeling, Stefan Jakobs and Roman Schmidt for fruitful discussions on various aspects of nanoscale microscopy.

This thesis greatly benefitted from the interdisciplinary scientific environment in the department. For the contributions from beyond the field of physics, I would like to thank

... Rebecca Medda, Christian Wurm, Tanja Gilat, Ellen Rothermel and Rita SchmitzSalue for support with the sample preparation and for general guidance in the biology labs.

... Mariano Bossi for the nanoparticles in the SMS project, Vladimir Belov and his 'Organic Chemistry Group' for synthesizing the switchable rhodamine amides and André C. Stiel, Martin Andresen and Sylvia Löbermann for providing rsFastLime.

... Marco Roose, Jaydev Jethwa, Harald Meyer and Donald Ouw for support in all aspects of laser technology, electronic circuits and computers.

... Rainer Pick for his excellent contributions to the design of the 4Pi setup and the SMS sample stage.

... Nicole Ehrmann for doing her diploma thesis on 4Pi microscopy. She carried out some of the measurements whose results are shown in chapter 3.

... Claas v. Middendorff, Daniel Aquino, Rebecca Medda, Christian Wurm, Jan Keller, Alexander Egner and Jaydev Jethwa for proof-reading (parts of) the manuscript.

My special thanks go to my parents for their keen interest and unconditional support, and last but not least, to Saskia for encouraging me and for kindly ignoring all the unpleasant aspects which come along during a $\mathrm{PhD}$-thesis. 



\section{Lebenslauf}

\section{Persönliche Daten}

$\begin{array}{ll}\text { Name, Vorname } & \text { Geisler, Claudia } \\ \text { Geburtsdatum } & \text { 28.08.1979 } \\ \text { Geburtsort } & \text { Hannover } \\ \text { Nationalität } & \text { deutsch }\end{array}$

Schulausbildung

1986-1990 Grundschule Am Welfenplatz, Hannover

1990-1992 Orientierungsstufe Anderten, Hannover

1992-1999 Gymnasium Schillerschule, Hannover

\section{Studium}

1999-2001

Grundstudium Allgemeine Physik, Leibniz Universität Hannover

2001-2005

2002-2003

Hauptstudium Allgemeine Physik, Leibniz Universität Hannover

seit 2005

Auslandsstudium, Lancaster University, Lancaster, Großbritannien

Promotion am Max-Planck-Institut für Biophysikalische Chemie,

Abteilung NanoBiophotonik 